\title{
A Mathematical Analysis of Casimir Interactions I: The Scalar Field
}

\author{
Yan-Long Fang and Alexander Strohmaier@
}

\begin{abstract}
Starting from the construction of the free quantum scalar field of mass $m \geq 0$, we give mathematically precise and rigorous versions of three different approaches to computing the Casimir forces between compact obstacles. We then prove that they are equivalent.
\end{abstract}

\section{Introduction}

Casimir interactions are forces between objects such as perfect conductors. They can be either understood as quantum fluctuations of the vacuum or as the total effect of van der Waals forces. Hendrik Casimir predicted and computed this effect in the special case of two planar conductors in 1948 using an infinite mode summation [1]. This force was measured experimentally by Sparnaay about 10 years later [2]. Since then also, more precise measurements have been performed with good agreement to the theoretical prediction of Casimir [3-6]. Other geometric situations, such as for example the Casimir force between a sphere and a plane, were also considered in precision experiments $[7,8]$.

The classical way to compute Casimir forces, and indeed the way it was done by Casimir himself, is by performing a zeta function regularisation of the vacuum energy. This has been carried out for a number of particular geometric situations (see [9-13] and references therein). Since this method requires knowledge of the spectrum of the Laplace operator in order to perform the analytic continuation, it has long been a very difficult problem to compute the Casimir force in a generic geometric situation even from a non-rigorous point of view. Already, it has been realised by quantum field theorists (see, e.g. [14-18]) that the Casimir force can also be understood by considering the renormalised stress-energy tensor of the electromagnetic field. This tensor is defined by

Y-L. Fang: Supported by Leverhulme Grant RPG-2017-329. 
comparing the induced vacuum states of the quantum field with boundary conditions and the free theory. Once the renormalised stress-energy tensor is mathematically defined, the computation of the Casimir energy density becomes a problem of spectral geometry (see, e.g. [19]) and numerical analysis. The renormalised stress-energy tensor and its relation to the Casimir effect can be understood at the level of rigour of axiomatic algebraic quantum field theory. Currently, this is still the subject of ongoing research in mathematical physics (see, e.g. [20]) in particular when it comes to the effect in curved backgrounds and fields that are not scalar, or in situations when the objects move.

Recently progress was made in the non-rigorous numerical computation of Casimir forces between objects (see for example [21-23]). This approach uses a formalism that relates the Casimir energy to a determinant computed from boundary layer operators. Such determinant formulae result in finite quantities that do not require further regularisation and have been obtained and justified in the physics literature [24-33]. This has not only resulted in more efficient numerical algorithms but also in various asymptotic formulae for the Casimir forces for large and small separations. Many of the justifications and derivations of these formulae are based on physics considerations of macroscopic properties of matter or of van der Waals forces. As such they often involve ill-defined path integrals. From a mathematical point of view, considerations that link the determinant formulae to the spectral approach initially taken by Casimir have been largely formal computations or involve ad hoc cut-offs and regularisation procedures. We note that in the Appendix of [30] it is proved correctly that the Fredholm determinant in the final formula of the Casimir energy is well defined. Only recently a mathematical justification of these formulae was given in [34], relating it to the trace of a linear combination of powers of the Laplace operator. The determinant formulae are directly related to the multi-reflection expansion of Balian and Duplantier [35] that also yields a finite Casimir energy. We mention [36] in the mathematical literature where the Casimir energy of a piston configuration is expressed in terms of the zeta regularised Fredholm determinant of the Dirichlet-to-Neumann operator.

Summarising, we list several ways to compute the Casimir force acting on a compact object that have been proposed and carried out:

(1) Using a total energy obtained in some way by regularising the spectrally defined zeta function. This can be done either directly or by first considering a compact problem in a box and then taking the adiabatic limit.

(2) By integrating the renormalised stress-energy tensor around any surface enclosing the object.

(3) Using formulae for the energy in terms of a determinant of boundary layer operator.

The list is non-exhaustive and other methods exist, such as for example the worldline approach (see, e.g. [37] and references). Here, we will restrict ourselves to the listed methods. 
The aim of the present paper is to establish, in the case of finitely many compact objects, the precise mathematical meaning of each of the listed methods for the case of the scalar field and then prove that they give the same answer for the force (not necessarily for the energy). The main tool to achieve this will be the relative stress-energy tensor. This tensor mimics the definition of the relative trace of [34] and seems to not have been defined or studied previously in the literature. Note that the renormalised stress tensor becomes unbounded and non-integrable $[16,38]$ near the boundaries of objects and this makes it unsuitable to compute the total energy component from the energy density $T_{00}$. In contrast, the relative stress-energy tensor is smooth up to the obstacles and is much more regular when considering boundary variations. This relative stress-energy tensor does not satisfy Dirichlet or Neumann boundary conditions and therefore integration by parts involves boundary contributions. The relative energy density can be defined entirely in terms of functional calculus of the Laplace operator. This relative energy density has been introduced in [34]. It was shown to be integrable and its integral can be interpreted as the trace of a certain operator. The main result of [34] states that this trace can be expressed as the determinant of an operator constructed from boundary layer operators, thus providing a rigorous justification of the method (3) linking it with method (1). To show the equivalence of methods (2) and (3) we must provide a formula for the variation of the relative energy when one of the objects is moved. To compute this variation, we prove and use a special case of the Hadamard variation formula ([39-41]) adapted to the non-compact setting. We then show that as a consequence of this formula that the variation of the total energy equals the surface integral of the spatial components of the relative stress-energy tensor (see Theorem 5.9). This surface integral is also equal to the surface integral over the renormalised tensor (see Theorem 5.10). We will now give a more precise formulation of our main result.

We consider $d$-dimensional Euclidean space with $d \geq 2$. Let $\mathcal{O}$ be a bounded open subset of $\mathbb{R}^{d}$ with smooth boundary such that the complement $\mathcal{E}=\mathbb{R}^{d} \backslash \overline{\mathcal{O}}$ is connected. The domain $\mathcal{O}$ will be assumed to consist of $N$ many connected components $\mathcal{O}_{1}, \ldots, \mathcal{O}_{N}$. The space $X=\mathbb{R}^{d} \backslash \partial \mathcal{O}$ therefore consists of the $N+1$-many connected components $\mathcal{O}_{1}, \ldots, \mathcal{O}_{N}, \mathcal{E}$. We think of $\mathcal{O}$ as obstacles placed in $\mathbb{R}^{d}$, and $\mathcal{E}$ then corresponds to the exterior region of these obstacles. The set $X \subset \mathbb{R}^{d}$ consists of the interior and the exterior of the obstacles, separated by $\partial \mathcal{O}$. See Fig. 1 for an example with three obstacles.

For the free scalar field of mass $m \geq 0$, let $T_{\text {ren }}$ be the renormalised stress-energy tensor as defined in Sect. 2.2. In QFT terms, this stress-energy tensor is equivalent to the usual stress-energy tensor obtained from canonical quantisation when normal ordering is used with respect to the free vacuum state. This is a smooth symmetric two-tensor away from $\partial \mathcal{O}$, but it is singular at $\partial \mathcal{O}$ and the integral of $T_{00}$ over $\mathcal{E}$ does not converge. Let $T_{\text {rel }}$ be the relative stress-energy tensor as given in Definition 3.3. The relative total energy $E_{\text {rel }}$ is defined as the integral of $\left(T_{\text {rel }}\right)_{00}(x)$ over $\mathbb{R}^{d}$ which can be shown to exist and to be equal the trace of a certain combination of operators. In case $m>0$, the regularised energy $E_{\text {reg }}$ is defined in Sect. 6, Definition 6.3 via zeta function 


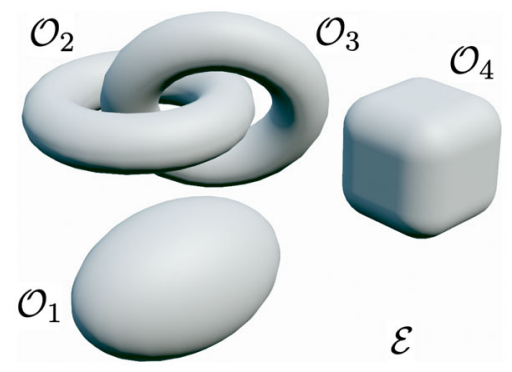

Figure 1. Configuration of four obstacles $\mathcal{O}_{1}, \mathcal{O}_{2}, \mathcal{O}_{3}, \mathcal{O}_{4}$ with complement $\mathcal{E}$ in a Euclidean space

regularisation. We would like to compute the force on an object $\mathcal{O}_{j}$ due to the presence of the other objects. Approach (2) is to directly compute

$$
F_{i}=\int_{\Sigma}\left(T_{\text {ren }}\right)_{i k} n^{k} \mathrm{~d} \sigma
$$

where $T_{\text {ren }}$ is the renormalised stress-energy tensor given in Definition 2.3 and $\Sigma$ is any smooth surface enclosing $\mathcal{O}_{j}$ (i.e. homologous to $\partial \mathcal{O}_{j}$ in $\overline{\mathcal{E}}$ ), $n^{k}$ is the exterior unit normal vector field, and $\mathrm{d} \sigma$ is the surface measure on $\Sigma$. If energy conservation holds, i.e. no energy is radiated off to infinity, one expects this force to be the directional derivative of the total energy when $\mathcal{O}_{j}$ is moved rigidly. Thus, let $Z$ be a constant vector field on $\mathbb{R}^{d}$. Let $Y$ be a vector field that equals $Z$ near $\mathcal{O}_{j}$ and vanishes near $\mathcal{O}_{l}$ when $l \neq j$. The vector field generates a flow $\Phi_{\epsilon}$ that, for $\epsilon$ near zero, moves the object $\mathcal{O}_{j}$ rigidly and we end up with a configuration that depends on the parameter $\epsilon$. The total energies $E_{\text {rel }}$ and $E_{\text {reg }}$ then also depend on $\epsilon$ in that way and become functions of $\epsilon$ in a small interval around zero. The change of these energies with respect to the flow can be interpreted as the change of energy needed to move object $\mathcal{O}_{j}$ rigidly relative to the other objects in the direction of $Z$. Our main result may be stated as follows.

Theorem 1.1. The relative energy $E_{\mathrm{rel}}(\epsilon)$ is differentiable in $\epsilon$ at $\epsilon=0$ and its derivative equals $F_{i} Z^{i}$, where

$$
F_{i}=\int_{\Sigma}\left(T_{\text {rel }}\right)_{i k} n^{k} \mathrm{~d} \sigma=\int_{\Sigma}\left(T_{\text {ren }}\right)_{i k} n^{k} \mathrm{~d} \sigma .
$$

Moreover,

$$
E_{\mathrm{rel}}=\frac{1}{\pi} \int_{m}^{\infty} \frac{\omega}{\sqrt{\omega^{2}-m^{2}}} \log \operatorname{det} Q_{\mathrm{rel}}(\omega) \mathrm{d} \omega,
$$

where $Q_{\mathrm{rel}}(\omega)=Q_{\mathrm{i} \omega}\left(\tilde{Q}_{\mathrm{i} \omega}\right)^{-1}$ is defined in detail in Sect. 3 and constructed out of boundary layer operators for the Laplacian. If $m>0$, then $E_{\mathrm{rel}}(\epsilon)-E_{\mathrm{reg}}(\epsilon)$ is constant near $\epsilon=0$.

We note that this mathematical theorem simply shows that all these proposed computational methods give the same Casimir interactions in the case of separated rigid bodies. The statement does not say anything about the actual 
origin of the Casimir force or its existence, which needs to be determined from experiments or physics considerations. There is however a strong argument for the expression

$$
F_{i}=\int_{\Sigma}\left(T_{\mathrm{rel}}\right)_{i k} n^{k} \mathrm{~d} \sigma
$$

to be a directly relevant physical quantity. Our point of view is that the stressenergy tensor does not have an absolute meaning in this context, but rather is used to compare two vacuum states (normal ordering depends on a comparison state). If we would like to know the effect for the rigid objects $\mathcal{O}_{2}, \ldots \mathcal{O}_{N}$ on the rigid object $\mathcal{O}_{1}$, the states to compare are not the ground state with Dirichlet conditions and the free ground state. It is rather the vacuum states obtained from the Laplacian with Dirichlet conditions imposed on $\mathcal{O}_{1}$ alone and with Dirichlet conditions on all the objects. The comparison of these two states yields a stress-energy tensor that is completely regular near $\mathcal{O}_{1}$, and the computation of the force based on this tensor leads directly to the above formula without regularisation.

The paper is organised as follows. In Sect. 2, we review the rigorous construction of the free scalar field of mass $m \geq 0$ in the presence of boundaries and show how this leads to a natural definition of the renormalised stress-energy tensor, which is given in Sect. 2.2. We also review its most important properties and express it in terms of spectral quantities for the Laplace operator. Section 3 introduces the relative setting and gives the definition of the relative stress-energy tensor and its basic properties. Some norm estimates on the relative resolvent are given in Sect. 4, which provides mathematical justifications for later proofs. In Sect. 5, we prove a Hadamard variation formula and compute the variation of the relative energy to establish the first part of the main theorem. In Sect. 6, we show that for $m>0$ the renormalised version of the zeta function has a meromorphic continuation and can thus be used to define the regularised energy. This section also contains a proof that variations of the regularised energy and the relative energy coincide. To illustrate the method and relate it to the classical computations we treat the easier example of the one-dimensional Casimir energy explicitly in Appendix 7. This example also illustrates that a divergence term for the time-component of the renormalised stress-energy tensor that is normally neglected needs to be taken into account to obtain the correct result (see Remark 2.5).

In a follow-up paper, we will establish a similar theorem for the electromagnetic field. We note here that the stress-energy for the electromagnetic field is quite different from the scalar field and there are additional complications such as zero modes [42] that are absent for the scalar field. Moreover, the boundary conditions for the electromagnetic field are slightly more complicated, and cannot be reduced to Dirichlet boundary conditions. We therefore decided to not attempt a unified treatment which would obscure the result by additional notations.

Our approach is expected to carry over to other boundary conditions such a Neumann, mixed Dirichlet-Neumann or Robin boundary conditions with the 
single-layer operators replaced by the appropriate layer operators. As in the electromagnetic case, additional technical problems need to be overcome in these cases due to the possible appearance of zero modes and singularities of the Dirichlet-to-Neumann map at zero.

\subsection{Notations}

Let $M \subset \mathbb{R}^{d}$ be an open subset. By the Schwartz kernel theorem, continuous linear operators $A: C_{0}^{\infty}(M) \rightarrow \mathcal{D}^{\prime}(M)$ are in one to one correspondence to distributions in $\mathcal{D}^{\prime}(M \times M)$, i.e. for every such $A$ there exists a unique Schwartz kernel in $\mathcal{D}^{\prime}(M \times M)$. In this paper, the Schwartz kernel of $A$ will be denoted by $\breve{A}$.

\section{Scalar Quantum Field Theory with Dirichlet Boundary Conditions}

Let $-\Delta$ be the (positive) Dirichlet Laplacian imposed on the codimension one submanifold $\partial \mathcal{O}$. By definition, this is the unbounded self-adjoint operator defined on the Hilbert space $L^{2}\left(\mathbb{R}^{d}\right)$ associated with the Dirichlet quadratic form $q_{D}(f, f)=\int_{X}\|\nabla f\|^{2} \mathrm{~d} x$ with form domain being the Sobolev space $H_{0}^{1}(X)$.

As a consequence of elliptic regularity $\left([43]^{*}\right.$ Sect. 7.2$)$, we have for the domain of $\operatorname{dom}\left((-\Delta)^{\frac{s}{2}}\right)$ equipped with its graph norm for any $s \in 2 \mathbb{N}_{0}$ the continuous inclusions

$$
H_{\text {comp }}^{s}(X) \subset \operatorname{dom}\left((-\Delta)^{\frac{s}{2}}\right) \subset H_{\mathrm{loc}}^{s}(X) .
$$

One can use complex interpolation $\left([44]^{*}\right.$ Sect. 4 and Theorem 4.2) to extend this to any $s \geq 0$. Here, $H_{\text {comp }}^{s}(X)$ denotes the space of functions in $H^{s}(X)$ with compact support in $X$.

The Hilbert space $L^{2}\left(\mathbb{R}^{d}\right)$ then decomposes into a direct sum

$$
L^{2}\left(\mathbb{R}^{d}\right)=L^{2}\left(\mathcal{O}_{1}\right) \oplus \ldots \oplus L^{2}\left(\mathcal{O}_{N}\right) \oplus L^{2}(\mathcal{E})
$$

and each subspace is an invariant subspace for $-\Delta$ in the sense that any bounded function of the operator as defined by spectral calculus will leave these subspaces invariant. The restriction of $-\Delta$ to $L^{2}\left(\mathcal{O}_{j}\right)$ is the Dirichlet Laplacian on the interior of $\mathcal{O}_{j}$ and therefore has compact resolvent. The restriction of $-\Delta$ to $L^{2}(\mathcal{E})$ has purely absolutely continuous spectrum $[0, \infty)$. By comparison, we also have the free Laplacian $-\Delta_{\text {free }}$ on $\mathbb{R}^{d}$ which corresponds to the case $\mathcal{O}=\varnothing$. Throughout the paper, we fix a mass parameter $m \geq 0$.

Definition 2.1. The relativistic Hamiltonian $H$ is defined to be the self-adjoint operator $H=\left(-\Delta+m^{2}\right)^{\frac{1}{2}}$.

The space-time $M$ we consider is the Lorentzian space-time $\mathbb{R} \times X$ with Minkowski metric. The forward and backward fundamental solutions $G_{+/-}$: 
$C_{0}^{\infty}(M) \rightarrow C^{\infty}(M)$ of the Klein-Gordon operator $\square+m^{2}$ with Dirichlet boundary conditions are given by

$$
\begin{aligned}
& \left(G_{+} f\right)(t, x)=\int\left(\theta\left(t-t^{\prime}\right) H^{-1} \sin \left(H\left(t-t^{\prime}\right)\right) f\left(t^{\prime}, \cdot\right)\right)(x) \mathrm{d} t^{\prime}, \\
& \left(G_{-} f\right)(t, x)=\int\left(\theta\left(t^{\prime}-t\right) H^{-1} \sin \left(H\left(t^{\prime}-t\right)\right) f\left(t^{\prime}, \cdot\right)\right)(x) \mathrm{d} t^{\prime},
\end{aligned}
$$

where $\theta$ is the Heaviside step function. As usual in canonical quantisation, one considers the difference $G=G_{+}-G_{-}$given by

$$
(G f)(t, x)=\int\left(H^{-1} \sin \left(H\left(t-t^{\prime}\right)\right) f\left(t^{\prime}, \cdot\right)\right)(x) \mathrm{d} t^{\prime} .
$$

Here, $H^{-1} \sin \left(H\left(t-t^{\prime}\right)\right)$ is defined by spectral calculus. Since the function $\left|x^{-1} \sin (x t)\right|$ is bounded by $|t|$, the operator $H^{-1} \sin \left(H\left(t-t^{\prime}\right)\right)$ defines for any $s \geq 0$ a bounded map from $H_{\text {comp }}^{s}(X)$ to $\operatorname{dom}(-\Delta)^{s} \subset H^{s}\left(\mathcal{O}_{1}\right) \oplus \ldots H^{s}\left(\mathcal{O}_{N}\right) \oplus$ $H^{s}(\mathcal{E})$. Here, the inclusion of the domain in the Sobolev spaces follows for $s \in 2 \mathbb{N}_{0}$ from elliptic regularity up to the boundary ( $[45]^{*}$ Theorem 4.18 ) and for general $s \geq 0$ by interpolation. In particular, this means that $H^{-1} \sin \left(H\left(t-t^{\prime}\right)\right)$ has a distributional integral kernel. We can define a symplectic structure $\sigma$ on $\mathcal{W}=C_{0}^{\infty}(M) /\left(\left(\square+m^{2}\right) C_{0}^{\infty}(M)\right)$ by

$$
\sigma([f],[g])=(f, G g) .
$$

This induces a symplectic structure on $G C_{0}^{\infty}(M)$ that is well known to coincide with the standard symplectic structure on the space of solutions. Indeed, if we define $u=G f$ and $v=G g$ for $f, g \in C_{0}^{\infty}(M)$ then $u$ and $v$ solve the KleinGordon equation with Dirichlet boundary conditions and

$$
(f, G g)=\int_{X}\left(\partial_{t} u\right) v-\left(\partial_{t} v\right) u \mathrm{~d} x .
$$

In this equality, the right-hand side is independent of $t$.

\subsection{Field Algebra and the Vacuum State}

The field algebra of the real Klein-Gordon field is then the (unbounded) CCR *-algebra of this symplectic space. Instead of using the symplectic space $\mathcal{W}$ one can describe this algebra $\mathcal{A}$ directly as the complex unital $*$-algebra generated by symbols $\phi(f)$ with $f \in C_{0}^{\infty}(M, \mathbb{R})$ satisfying the relation

$$
\begin{aligned}
& f \rightarrow \phi(f) \quad \text { is real linear, } \\
& {\left[\phi\left(f_{1}\right), \phi\left(f_{2}\right)\right]=-\mathrm{i}\left(f_{1}, G f_{2}\right) \mathbf{1}} \\
& \phi(f)^{*}=\phi(f), \\
& \phi\left(\left(\square+m^{2}\right) f\right)=0 .
\end{aligned}
$$

Physical states of this quantum system are states on this $\star$-algebra. The construction and physical interpretations of such states usually relies on a Fock representation of $\mathcal{A}$. This representation is chosen on physical grounds as a positive energy representation.

We briefly explain this now. The group of time translations $\left(T_{s} f\right)(t, x)=$ $f(t-s, x)$ commutes with $\square+m^{2}$ and $G$ and therefore $\alpha_{t}(\phi(f)):=\phi\left(T_{t} f\right)$ defines a group of $*$-automorphisms of $\mathcal{A}$. If a state $\omega: \mathcal{A} \rightarrow \mathbb{C}$ is invariant then 
this group lifts to a group of unitary transformations $U(t)$ on the GNS-Hilbert space which is uniquely determined by

$$
\begin{aligned}
& \pi\left(\alpha_{t}(a)\right)=U_{t} \pi(a) U_{-t}, \\
& U_{t} \Omega=\Omega .
\end{aligned}
$$

We say that $\pi$ is a positive energy representation if this group is strongly continuous and its infinitesimal generator has non-negative spectrum.

We will focus in this paper on the quasi-free ground state. This means that the state is completely determined by its two point distribution

$$
\omega_{2} \in \mathcal{D}^{\prime}(M \times M), \quad \omega_{2}\left(f_{1} \otimes f_{2}\right)=\omega\left(\phi\left(f_{1}\right) \phi\left(f_{2}\right)\right)
$$

which is given explicitly as

$$
\omega_{2}\left(f_{1} \otimes f_{2}\right)=\int_{\mathbb{R} \times \mathbb{R}}\left(f_{1}(t, \cdot), \frac{1}{2} H^{-1} e^{-\mathrm{i} H\left(t-t^{\prime}\right)} f_{2}\left(t^{\prime}, \cdot\right)\right) \mathrm{d} t \mathrm{~d} t^{\prime},
$$

i.e. $\omega_{2}$ is the integral kernel of the operator $H^{-1} e^{-\mathrm{i} H\left(t-t^{\prime}\right)}$. In case $m=0$, the spectrum of $H$ contains zero and this expression needs to be interpreted in the sense of quadratic forms. Namely, it follows from general resolvent expansions (for example [42]*Theorems $1.5,1.6$ and 1.7) that $C_{0}^{\infty}(X)$ is contained in the domain of the operator $H^{-\frac{1}{2}}$. This follows from the formula

$$
\left\langle H^{-\frac{1}{2}} f, H^{-\frac{1}{2}} f\right\rangle=\frac{2}{\pi} \int_{0}^{\infty}\left\langle\left(-\Delta+\lambda^{2}\right)^{-1} f, f\right\rangle \mathrm{d} \lambda .
$$

In particular, the space of test functions $C_{0}^{\infty}(X)$ is contained in the form domain of $H^{-1}$ and therefore, by the Schwartz kernel theorem, the operator $H^{-1}$ has a distributional kernel in $\mathcal{D}^{\prime}(X \times X)$. This is of course also the case for general $m>0$. We will denote the integral kernel of $H^{-1}$ by $\breve{H}^{-1}$, mildly abusing notation.

One can check directly that $\omega_{2}$ defines a positive energy representation. Instead of using $-\Delta$, we could also have used $-\Delta_{\text {free }}$, the free Laplace operator. This also defines a positive energy state on the free algebra of observables $\mathcal{A}_{\text {free }}$ which we will denote by $\omega_{\text {free }}$, and similarly we use the notation $H_{\text {free }}=$ $\left(-\Delta_{\text {free }}+m^{2}\right)^{\frac{1}{2}}$. There states can be compared by restricting them to certain subalgebras that are contained in both the algebra of observables and the free algebra of observables. For example if $U$ is contained in a double cone in $M$ then $\mathcal{A}(U)$ which is generated by $\phi(f), \operatorname{supp}(f) \subset U$ can be thought of as a subset of both $\mathcal{A}$ and $\mathcal{A}_{\text {free }}$ and therefore both states can be restricted to this algebra.

\subsection{The Renormalised Stress-energy Tensor}

The classical stress-energy tensor of the Klein-Gordon field for a smooth realvalued solution $u$ is given by

$$
T^{c l}(u)=\mathrm{d} u \otimes \mathrm{d} u-\frac{1}{2} g(\mathrm{~d} u, \mathrm{~d} u) g-\frac{1}{2} g m^{2} u^{2} .
$$

This is a symmetric 2-tensor on $M$ and one can easily show, using the KleinGordon equation, that it is divergence-free. Here, $g$ is the Minkowski metric on $M$ with signature $(-1,1, \ldots, 1)$. The Euclidean metric on $\mathbb{R}^{d}$ will be denoted 
by $h$. The components $T_{i j}^{c l}$ of the stress-energy tensor are the restrictions to the diagonal of the functions $Q_{i j}^{c l}(x, y)$ defined on $M \times M$ by

$Q_{i j}^{c l}(x, y)=\left(\nabla_{i} u\right)(x) \otimes\left(\nabla_{j} u\right)(y)-\frac{1}{2} g_{i j}\left(\nabla_{k} u\right)(x) \cdot \nabla^{k} u(y)-\frac{1}{2} g_{i j} m^{2} u(x) u(y)$.

The quantum field theory counterpart of $Q$ can be written in the field algebra as a field-algebra-valued bilinear form in the test functions $f_{1}, f_{2} \in C^{\infty}(M)$ as $Q_{i j}\left(f_{1} \otimes f_{2}\right)=\phi\left(\nabla_{i} f_{1}\right) \phi\left(\nabla_{j} f_{2}\right)-\frac{1}{2} \phi\left(\nabla_{k} f_{1}\right) \phi\left(\nabla^{k} f_{2}\right) g_{i j}-\frac{1}{2} g_{i j} m^{2} \phi\left(f_{1}\right) \phi\left(f_{2}\right)$.

The expectation value of $Q_{i j}$ with respect to the state $\omega$ is then given in terms of the two point function $\omega_{2}$ as

$$
\omega\left(Q_{i j}\right)\left(f_{1}, f_{2}\right)=\omega_{2}\left(\nabla_{i} f_{1} \otimes \nabla_{j} f_{2}-\frac{1}{2} \nabla_{k} f_{1} \otimes \nabla^{k} f_{2} g_{i j}-\frac{1}{2} g_{i j} m^{2} f_{1} \otimes f_{2}\right) .
$$

Let $K(t, x, y)$ be the distributional kernel of $\frac{1}{2} H^{-1} e^{-\mathrm{i} H t}$. Then, the distribution $\omega\left(Q_{i j}\right)$ with respect to the ground state is given by

$$
\left\{\begin{array}{l}
\omega\left(Q_{00}\right)\left(t, t^{\prime}, x, x^{\prime}\right)=\frac{1}{2}\left(\partial_{t} \partial_{t^{\prime}}+\sum_{k=1}^{d} \partial_{x^{k}} \partial_{x^{\prime k}}+m^{2}\right) K\left(t-t^{\prime}, x, x^{\prime}\right) \\
\omega\left(Q_{0 i}\right)\left(t, t^{\prime}, x, x^{\prime}\right)=\left(\partial_{t^{\prime}} \partial_{x^{i}}\right) K\left(t-t^{\prime}, x, x^{\prime}\right) \text { for } 1 \leq i \leq d \\
\omega\left(Q_{i j}\right)\left(t, t^{\prime}, x, x^{\prime}\right)=\left(\partial_{x^{\prime i}} \partial_{x^{j}}\right) K\left(t-t^{\prime}, x, x^{\prime}\right) \text { for } i \neq j \quad \text { and } \quad 1 \leq i, j \leq d \\
\omega\left(Q_{i i}\right)\left(t, t^{\prime}, x, x^{\prime}\right)=\frac{1}{2}\left(\partial_{t} \partial_{t^{\prime}}+\partial_{x^{i}} \partial_{x^{\prime i}}-\sum_{k \neq i}^{d} \partial_{x^{k}} \partial_{x^{\prime k}}-m^{2}\right) K\left(t-t^{\prime}, x, x^{\prime}\right) \text { for } 1 \leq i \leq d
\end{array}\right.
$$

or in short,

$$
\omega\left(Q_{i j}\right)\left(t, t^{\prime}, x, x^{\prime}\right)=\left[\partial_{i}^{\prime} \partial_{j} K-\frac{1}{2} g_{i j}\left(\partial_{k}^{\prime} \partial^{k}+m^{2}\right) K\right]\left(t-t^{\prime}, x, x^{\prime}\right) .
$$

The above expressions are formal and make sense only when paired with test functions. We will use such formal notation throughout the paper when there is danger of confusion. The expectation value of the stress-energy tensor would correspond to the restriction of $\omega\left(Q_{i j}\right)$ to the diagonal as a distribution. Unfortunately, the distribution $\omega\left(Q_{i j}\right)$ is singular and cannot be restricted to the diagonal in a straightforward manner. If one is interested in relative quantities only then one can still define the renormalised expectation value of the stress-energy tensor between the states. Both states $\omega$ and $\omega_{\text {free }}$ are positive energy states and therefore satisfy the Hadamard condition (for example [46]*Theorem 6.3). By uniqueness of such Hadamard states, the difference of the two-point distributions is smooth near the diagonal in $M \times M$. In the present case, this can also be seen more directly as follows. Let $K_{\text {free }}(t, x, y)$ be the kernel of $\frac{1}{2} H_{\text {free }}^{-1} e^{-\mathrm{i} H_{\text {free }} t}$. We will consider the difference $\mathcal{K}(t, x, y)=K(t, x, y)-K_{\text {free }}(t, x, y)$.

Theorem 2.2. The distribution $\mathcal{K}$ is smooth near the set $\{(0, x, y) \mid x, y \in X\} \subset$ $\mathbb{R} \times X \times X$. In particular $\breve{H}^{-1}-\breve{H}_{\text {free }}^{-1}$ is smooth in $X \times X$.

Proof. The distribution $\mathcal{K}(t, x, y)$ is a solution of the wave equation

$$
\partial_{t}^{2}-\frac{1}{2}\left(\Delta_{x}+\Delta_{y}\right) \mathcal{K}(t, x, y)=0
$$


on $\mathbb{R} \times X \times X$ with initial conditions

$$
\begin{aligned}
& \mathcal{K}(0, x, y)=\frac{1}{2}\left(\breve{H}^{-1}(x, y)-\breve{H}_{\text {free }}^{-1}(x, y)\right), \\
& \left.\partial_{t} \mathcal{K}(t, x, y)\right|_{t=0}=0 .
\end{aligned}
$$

By [34]*Lemma 5.1, integral kernel $\kappa_{\lambda}$ of the resolvent difference

$$
\left(-\Delta+\lambda^{2}\right)^{-1}-\left(-\Delta_{\text {free }}+\lambda^{2}\right)^{-1}
$$

is smooth and satisfies on any compact subset $U$ of $X \times X$ a $C^{k}$-norm bound of the form

$$
\left\|\kappa_{\lambda}\right\|_{C^{k}(U)} \leq C_{k, U}|\log (|\lambda|)|^{\ell} e^{-\delta^{\prime} \lambda} \text { for all } \lambda>0
$$

for some $\ell \geq 0$ and $\delta^{\prime}>0$. Therefore, the integral representation

$$
H^{-1}-H_{\text {free }}^{-1}=\frac{2}{\pi} \int_{m}^{\infty} \frac{\lambda}{\sqrt{\lambda^{2}-m^{2}}} \kappa_{\lambda} \mathrm{d} \lambda
$$

converges in the $C^{\infty}(X \times X)$ topology. Thus, the distribution $\breve{H}^{-1}-\breve{H}_{\text {free }}^{-1}$ is smooth in $X \times X$. Since the initial conditions are smooth the solution $\mathcal{K}$ is smooth where it is uniquely determined by the initial data. This is the case in a neighbourhood of $t=0$ in $\mathbb{R} \times X \times X$.

Hence, the distribution $\omega\left(Q_{i j}\right)-\omega_{\text {free }}\left(Q_{i j}\right)$ is a smooth function in a neighbourhood of the diagonal $\Delta \subset M \times M$.

Definition 2.3. The components $\left(T_{\text {ren }}\right)_{i j}$ of the renormalised stress-energy tensor $T_{\text {ren }}$ are defined to be the restriction to the diagonal of the function $\omega\left(Q_{i j}\right)-\omega_{\text {free }}\left(Q_{i j}\right)$.

Theorem 2.4. The renormalised stress-energy tensor is symmetric and is given by

$$
\left\{\begin{array}{l}
\left(T_{\text {ren }}\right)_{00}=\left.\frac{1}{2}\left(\breve{H}-\breve{H}_{\text {free }}\right)\right|_{\Delta}+\frac{1}{8} \Delta\left[\left.\left(\breve{H}^{-1}-\breve{H}_{\text {free }}^{-1}\right)\right|_{\Delta}\right] \\
\left(T_{\text {ren }}\right)_{i j}=\left.\frac{1}{2}\left[\partial_{i} \partial_{j}^{\prime}\left(\breve{H}^{-1}-\breve{H}_{\text {free }}^{-1}\right)\right]\right|_{\Delta}-\frac{1}{8} h_{i j} \Delta\left[\left.\left(\breve{H}^{-1}-\breve{H}_{\text {free }}^{-1}\right)\right|_{\Delta}\right] \\
\left(T_{\text {ren }}\right)_{0 i}=0
\end{array}\right.
$$

and

$$
\left\{\begin{array}{l}
\left(T_{\text {ren }}\right)_{00}=\left.\frac{1}{2}\left(\breve{H}-\breve{H}_{\text {free }}\right)\right|_{\Delta}+\frac{1}{8} \Delta\left[\left.\left(\breve{H}^{-1}-\breve{H}_{\text {free }}^{-1}\right)\right|_{\Delta}\right] \\
\left(T_{\text {ren }}\right)_{i j}=-\left.\frac{1}{2}\left[\partial_{i} \partial_{j}\left(\breve{H}^{-1}-\breve{H}_{\text {free }}^{-1}\right)\right]\right|_{\Delta}+\frac{1}{4} \partial_{i} \partial_{j}\left[\left.\left(\breve{H}^{-1}-\breve{H}_{\text {free }}^{-1}\right)\right|_{\Delta}\right]-\frac{1}{8} h_{i j} \Delta\left[\left.\left(\breve{H}^{-1}-\breve{H}_{\text {free }}^{-1}\right)\right|_{\Delta}\right] \\
\left(T_{\text {ren }}\right)_{0 i}=0
\end{array}\right.
$$

for $1 \leq i, j \leq d$. Note that here $\breve{H}^{-1}$ and $\breve{H}_{\text {free }}^{-1}$ are the integral kernels of $H^{-1}$ and $H_{\text {free }}^{-1}$, respectively. Moreover, the expression $\left.\breve{A}\right|_{\Delta}$ means the restriction of the integral kernel, $\breve{A}$, to the diagonal (See Sect. 1.1). 
Remark 2.5. The terms of divergence forms in the renormalised stress-energy tensor are commonly neglected in the literature, as one may naively think that they have zero contribution when integrating over the whole space. However, this is not the case. As it is not integrable due to the singular behaviour near the boundary, the divergence theorem does not apply in this case. See Appendix 7 for the simplest case. The problem disappears when we work with the relative stress-energy tensor given in Definition 3.3.

Proof of Theorem 2.4. Let $\mathcal{K}\left(t, x, x^{\prime}\right)$ be the kernel of $\frac{1}{2}\left(H^{-1} e^{-\mathrm{i} H t}-H_{\text {free }}^{-1} e^{-\mathrm{i} H_{\text {free }} t}\right)$ and $\mathscr{K}\left(t, t^{\prime}, x, x^{\prime}\right)=\mathcal{K}\left(t-t^{\prime}, x, x^{\prime}\right)$. We have that

$$
\left(T_{\text {ren }}\right)_{i j}=\left.\left[\partial_{i}^{\prime} \partial_{j}-\frac{1}{2} g_{i j}\left(\partial_{k}^{\prime} \partial^{k}+m^{2}\right)\right] \mathscr{K}\right|_{\Delta},
$$

where $\partial_{0}=\partial_{t}, \partial_{0}^{\prime}=\partial_{t^{\prime}}, \partial_{i}=\partial_{x^{i}}$ and $\partial_{i}^{\prime}=\partial_{x^{\prime}}$ for $1 \leq i \leq d$. By theorem 2.2 the distribution $\mathscr{K}\left(t, t^{\prime}, x, x^{\prime}\right)$ is smooth in a neighbourhood of the diagonal $\Delta \subset M \times M$. Moreover, $\mathcal{K}\left(t-t^{\prime}, \cdot, \cdot\right)$ is the kernel of a symmetric operator on $X$ with respect to the real inner product $(\cdot, \cdot)$ and therefore satisfies

$$
\mathcal{K}\left(t-t^{\prime}, x, x^{\prime}\right)=\mathscr{K}\left(t, t^{\prime}, x, x^{\prime}\right)=\mathscr{K}\left(t, t^{\prime}, x^{\prime}, x\right)=\mathcal{K}\left(t-t^{\prime}, x^{\prime}, x\right) .
$$

This implies

$$
\left\{\begin{array}{l}
\partial_{0} \mathscr{K}\left(t, t^{\prime}, x, x^{\prime}\right)=-\partial_{0}^{\prime} \mathscr{K}\left(t, t^{\prime}, x, x^{\prime}\right) \\
\partial_{i} \mathscr{K}\left(t, t^{\prime}, x, x^{\prime}\right)=\partial_{i}^{\prime} \mathscr{K}\left(t, t^{\prime}, x^{\prime}, x\right) \\
\partial_{i} \partial_{j} \mathscr{K}\left(t, t^{\prime}, x, x^{\prime}\right)=\partial_{i}^{\prime} \partial_{j}^{\prime} \mathscr{K}\left(t, t^{\prime}, x^{\prime}, x\right) \\
\partial_{i} \partial_{j}^{\prime} \mathscr{K}\left(t, t^{\prime}, x, x^{\prime}\right)=\partial_{i}^{\prime} \partial_{j} \mathscr{K}\left(t, t^{\prime}, x^{\prime}, x\right)
\end{array} \text { for } \quad 1 \leq i, j \leq d .\right.
$$

Using product rules, we have

$$
\partial_{i}\left(\left.\mathscr{K}\right|_{\Delta}\right)=\left.\left(\partial_{i} \mathscr{K}\right)\right|_{\Delta}+\left.\left(\partial_{i}^{\prime} \mathscr{K}\right)\right|_{\Delta},
$$

which gives

$$
\partial_{i} \partial_{j}\left(\left.\mathscr{K}\right|_{\Delta}\right)=\left.\left(\partial_{i} \partial_{j} \mathscr{K}\right)\right|_{\Delta}+\left.\left(\partial_{i} \partial_{j}^{\prime} \mathscr{K}\right)\right|_{\Delta}+\left.\left(\partial_{j} \partial_{i}^{\prime} \mathscr{K}\right)\right|_{\Delta}+\left.\left(\partial_{i}^{\prime} \partial_{j}^{\prime} \mathscr{K}\right)\right|_{\Delta}
$$

That is

$$
\left.\left(\partial_{i} \partial_{j}^{\prime} \mathscr{K}\right)\right|_{\Delta}=\partial_{i} \partial_{j}\left(\left.\mathscr{K}\right|_{\Delta}\right)-\left.\left(\partial_{i} \partial_{j} \mathscr{K}\right)\right|_{\Delta}-\left.\left(\partial_{j} \partial_{i}^{\prime} \mathscr{K}\right)\right|_{\Delta}-\left.\left(\partial_{i}^{\prime} \partial_{j}^{\prime} \mathscr{K}\right)\right|_{\Delta} \cdot
$$

Applying Eq. (8) to (10), we have

$$
\left\{\begin{array}{l}
\left.\left(\partial_{0} \partial_{0}^{\prime} \mathscr{K}\right)\right|_{\Delta}=-\left.\left(\partial_{0} \partial_{0} \mathscr{K}\right)\right|_{\Delta}=\left.\left(\breve{H}-\breve{H}_{\text {free }}\right)\right|_{\Delta} \\
\left.\left(\partial_{0} \partial_{i}^{\prime} \mathscr{K}\right)\right|_{\Delta}=-\left.\left(\partial_{0}^{\prime} \partial_{i}^{\prime} \mathscr{K}\right)\right|_{\Delta}=\left.\left(\partial_{0} \partial_{i} \mathscr{K}\right)\right|_{\Delta}=-\left.\left(\partial_{i} \partial_{0}^{\prime} \mathscr{K}\right)\right|_{\Delta} \quad \text { for } \quad 1 \leq i \leq d \\
\left.\left(\partial_{i} \partial_{j}^{\prime} \mathscr{K}\right)\right|_{\Delta}=\frac{1}{2} \partial_{i} \partial_{j}\left(\left.\mathscr{K}\right|_{\Delta}\right)-\left.\left(\partial_{i} \partial_{j} \mathscr{K}\right)\right|_{\Delta} \quad \text { for } \quad 1 \leq i, j \leq d
\end{array}\right.
$$

From Eqs. (8) and (9), we have

$$
\left.2 \partial_{i} \partial_{0} \mathscr{K}\right|_{\Delta}=\left.\partial_{i} \partial_{0} \mathscr{K}\right|_{\Delta}+\left.\partial_{i}^{\prime} \partial_{0} \mathscr{K}\right|_{\Delta}=\partial_{i}\left(\left.\partial_{0} \mathscr{K}\right|_{\Delta}\right)=0 \text { for } 1 \leq i \leq d .
$$


In other words, $\left(T_{\text {ren }}\right)_{0 i}=\left(T_{\text {ren }}\right)_{i 0}=0$ for $1 \leq i \leq d$. Hence, equations $(6),(11)$ and (12) show that $\left(T_{\text {ren }}\right)_{i j}$ is symmetric tensor on $M$. Moreover,

$$
\left(T_{\text {ren }}\right)_{i j}=\frac{1}{2} \partial_{i} \partial_{j}\left(\left.\mathscr{K}\right|_{\Delta}\right)-\left.\left(\partial_{i} \partial_{j} \mathscr{K}\right)\right|_{\Delta} \quad \text { for } \quad 1 \leq i \neq j \leq d
$$

and

$$
\begin{aligned}
\left(T_{\text {ren }}\right)_{i i}= & {\left.\left[\partial_{i}^{\prime} \partial_{i}-\frac{1}{2} g_{i i}\left(\partial_{k}^{\prime} \partial^{k}+m^{2}\right)\right] \mathscr{K}\right|_{\Delta} } \\
= & \frac{1}{2} \partial_{i} \partial_{i}\left(\left.\mathscr{K}\right|_{\Delta}\right)-\left.\left(\partial_{i} \partial_{i} \mathscr{K}\right)\right|_{\Delta} \\
& \quad-\frac{1}{2} g_{i i}\left(\frac{1}{2} \partial_{k} \partial^{k}\left(\left.\mathscr{K}\right|_{\Delta}\right)-\left.\left(\partial_{k} \partial^{k} \mathscr{K}-m^{2} \mathscr{K}\right)\right|_{\Delta}\right) .
\end{aligned}
$$

Since $\partial_{k} \partial^{k} \mathscr{K}-m^{2} \mathscr{K}=0$, we have

$$
\left(T_{\text {ren }}\right)_{i i}=\frac{1}{2} \partial_{i} \partial_{i}\left(\left.\mathscr{K}\right|_{\Delta}\right)-\left.\left(\partial_{i} \partial_{i} \mathscr{K}\right)\right|_{\Delta}-\frac{1}{4} g_{i i} \partial_{k} \partial^{k}\left(\left.\mathscr{K}\right|_{\Delta}\right) .
$$

When $i=0$,

$$
\left(T_{\text {ren }}\right)_{00}=\left.\frac{1}{2}\left(\breve{H}-\breve{H}_{\text {free }}\right)\right|_{\Delta}+\frac{1}{8} \Delta\left[\left.\left(\breve{H}^{-1}-\breve{H}_{\text {free }}^{-1}\right)\right|_{\Delta}\right]
$$

Also, we have

$$
\left.\left(\partial_{i} \partial_{j} \mathscr{K}\right)\right|_{\Delta}=\left.\frac{1}{2}\left[\partial_{i} \partial_{j}\left(\breve{H}^{-1}-\breve{H}_{\text {free }}^{-1}\right)\right]\right|_{\Delta} \quad \text { for } \quad 1 \leq i, j \leq d,
$$

which yields the expressions for the renormalised stress-energy tensor.

Theorem 2.6. The renormalised stress-energy tensor is divergence-free and independent of $t$.

Proof. Let $\mathcal{K}(t, x, y)$ and $\mathscr{K}\left(t, t^{\prime}, x, y\right)$ be the same as the in the previous theorem. Recall that the shorthand expression of (3) is given by

$$
\omega\left(Q_{i j}\right)\left(t, t^{\prime}, x, x^{\prime}\right)=\left[\partial_{i}^{\prime} \partial_{j} \mathscr{K}-\frac{1}{2} g_{i j}\left(\partial_{k}^{\prime} \partial^{k}+m^{2}\right) \mathscr{K}\right]\left(t, t^{\prime}, x, x^{\prime}\right) .
$$

Then, we have

$$
\left(T_{\text {ren }}\right)_{i j}(t, x)=\left.\omega\left(Q_{i j}\right)\right|_{\Delta}=\left.\left[\partial_{i}^{\prime} \partial_{j} \mathscr{K}-\frac{1}{2} g_{i j}\left(\partial_{k}^{\prime} \partial^{k}+m^{2}\right) \mathscr{K}\right]\right|_{\Delta} .
$$

Now we use product rules to get

$$
\partial_{i_{0}}\left(\left.\partial_{i_{1}} \cdots \partial_{i_{m}} \mathscr{K}\right|_{\Delta}\right)=\left.\left(\partial_{i_{0}} \partial_{i_{1}} \cdots \partial_{i_{m}} \mathscr{K}\right)\right|_{\Delta}+\left.\left(\partial_{i_{0}}^{\prime} \partial_{i_{1}} \cdots \partial_{i_{m}} \mathscr{K}\right)\right|_{\Delta} .
$$

In particular, we have

$$
\partial_{0}\left(\left.\partial_{i_{1}} \cdots \partial_{i_{m}} \mathscr{K}\right|_{\Delta}\right)=0
$$

Hence, one has

$$
\partial_{0}\left(T_{\text {ren }}\right)_{i j}(t, x)=\partial_{0}\left\{\left.\left[\partial_{i}^{\prime} \partial_{j} \mathscr{K}-\frac{1}{2} g_{i j}\left(\partial_{k}^{\prime} \partial^{k}+m^{2}\right) \mathscr{K}\right]\right|_{\Delta}\right\}=0,
$$

which means $\left(T_{\text {ren }}\right)_{i j}$ is independent of time. 
We use the same trick to show that

$$
\begin{aligned}
& \partial^{i}\left(T_{\text {ren }}\right)_{i j}(t, x)=\partial^{i}\left\{\left.\left[\begin{array}{ll}
\partial_{i}^{\prime} \partial_{j} \mathscr{K} & -\frac{1}{2} g_{i j}\left(\partial_{k}^{\prime} \partial^{k}+m^{2}\right) \mathscr{K}
\end{array}\right]\right|_{\Delta}\right\} \\
& \quad=\left.\left(\partial^{i} \partial_{i}^{\prime} \partial_{j} \mathscr{K}+\partial^{i} \partial_{i}^{\prime} \partial_{j} \mathscr{K}-\frac{1}{2} \partial_{j} \partial_{k}^{\prime} \partial^{k} \mathscr{K}-\frac{1}{2} \partial_{j}^{\prime} \partial_{k}^{\prime} \partial^{k} \mathscr{K}-\frac{1}{2} m^{2} \partial_{j} \mathscr{K}-\frac{1}{2} m^{2} \partial_{j}^{\prime} \mathscr{K}\right)\right|_{\Delta} .
\end{aligned}
$$

Now use the symmetric properties of $\mathscr{K}$ in Eqs. (7) and (8), we have

$$
\begin{aligned}
& \left.\left(\partial^{i} \partial_{i}^{\prime} \partial_{j} \mathscr{K}+\partial^{\prime i} \partial_{i}^{\prime} \partial_{j} \mathscr{K}-\frac{1}{2} \partial_{j} \partial_{k}^{\prime} \partial^{k} \mathscr{K}-\frac{1}{2} \partial_{j}^{\prime} \partial_{k}^{\prime} \partial^{k} \mathscr{K}-\frac{1}{2} m^{2} \partial_{j} \mathscr{K}-\frac{1}{2} m^{2} \partial_{j}^{\prime} \mathscr{K}\right)\right|_{\Delta} \\
& \quad=\left.\left(\partial^{\prime i} \partial_{i}^{\prime} \partial_{j} \mathscr{K}-\frac{1}{2} m^{2} \partial_{j} \mathscr{K}-\frac{1}{2} m^{2} \partial_{j}^{\prime} \mathscr{K}\right)\right|_{\Delta} .
\end{aligned}
$$

The function $\mathscr{K}$ solves the Klein-Gordon equation, i.e.

$$
\left(\partial^{\prime i} \partial_{i}^{\prime}-m^{2}\right) \mathscr{K}=\left(\partial^{i} \partial_{i}-m^{2}\right) \mathscr{K}=0 .
$$

Altogether, we have

$$
\partial^{i}\left(T_{\text {ren }}\right)_{i j}(t, x)=\left.\frac{1}{2}\left(\partial^{\prime i} \partial_{i}^{\prime} \partial_{j} \mathscr{K}-m^{2} \partial_{j} \mathscr{K}+\partial^{i} \partial_{i} \partial_{j}^{\prime} \mathscr{K}-m^{2} \partial_{j}^{\prime} \mathscr{K}\right)\right|_{\Delta}=0,
$$

which shows the divergence-free property of the renormalised stress-energy tensor.

\section{The Relative Trace-formula and the Casimir Energy}

As mentioned in the introduction the renormalised stress-energy tensor $T_{\text {ren }}(x)$ becomes unbounded and non-integrable when $x$ approaches the boundary of obstacles $[14,16,19,38]$. This prevents us from defining a renormalised total energy. One way to circumvent the problem is to introduce the relative framework of [34] which we now summarise. The main advantage of this construction is that it completely avoids ill-defined quantities and the need for regularisation.

Relative quantities are defined with respect to different obstacle configurations where instead of $\mathcal{O}$ only one obstacle $\mathcal{O}_{j}$ is present, i.e. where $\mathcal{O}$ is replaced by $\mathcal{O}_{j}$. If an operator is defined with respect to such a configuration we use the subscript $\mathcal{O}_{j}$, and we use the subscript $\mathcal{O}$ to distinguish it from the original configuration. For instance the renormalised stress-energy tensor $T_{\text {ren }}$ in Theorem 2.4 will be denoted by $\left(T_{\text {ren }}\right)_{\mathcal{O}}$, which shows its dependence on the presence of obstacles $\mathcal{O}=\mathcal{O}_{1} \cup \cdots \cup \mathcal{O}_{N}$. Similarly, $\left(T_{\text {ren }}\right)_{\mathcal{O}_{j}}$ denotes the renormalised stress-energy tensor when only obstacle $\mathcal{O}_{j}$ is present and $\Delta_{\mathcal{O}_{j}}$ denotes the Laplace operator with Dirichlet boundary condition on $\partial \mathcal{O}_{j}$. The operator $H_{\mathcal{O}_{j}}$ is defined in the same way.

Now we introduce a relative operator

$$
H_{\text {rel }}:=H_{\mathcal{O}}-H_{\text {free }}-\sum_{i=1}^{N}\left(H_{\mathcal{O}_{i}}-H_{\text {free }}\right) .
$$


More generally one defines the relative operator associated with a polynomially bounded function $f:[0, \infty) \rightarrow \mathbb{R}$, i.e.

$$
\begin{aligned}
D_{f, m}:= & f\left(\left(-\Delta_{\mathcal{O}}+m^{2}\right)^{\frac{1}{2}}\right)-f\left(\left(-\Delta_{\text {free }}+m^{2}\right)^{\frac{1}{2}}\right) \\
& -\sum_{i=1}^{N}\left(f\left(\left(-\Delta_{\mathcal{O}_{i}}+m^{2}\right)^{\frac{1}{2}}\right)-f\left(\left(-\Delta_{\text {free }}+m^{2}\right)^{\frac{1}{2}}\right)\right) .
\end{aligned}
$$

Since all our operators are densely defined operators on the same Hilbert space $L^{2}\left(\mathbb{R}^{d}\right)$ this combination makes sense. As a consequence of $f$ being polynomially bounded, the space $C_{0}^{\infty}(X)$ is in the domain of $D_{f}$ and therefore $D_{f}$ has a distribution kernel in $\mathcal{D}^{\prime}(X \times X)$.

To simplify our analysis later, we absorb the dependence of mass $m$ in the functional $f$. We could write $f_{m}$ to emphasise the dependence on $m$, but the later analysis will not be affected by $m$. Therefore, we omit the $m$-dependence and have

$$
D_{f}:=f\left(\left(-\Delta_{\mathcal{O}}\right)^{\frac{1}{2}}\right)-f\left(\left(-\Delta_{\text {free }}\right)^{\frac{1}{2}}\right)-\sum_{i=1}^{N}\left(f\left(\left(-\Delta_{\mathcal{O}_{i}}\right)^{\frac{1}{2}}\right)-f\left(\left(-\Delta_{\text {free }}\right)^{\frac{1}{2}}\right)\right) .
$$

The main result of [34] is that for a large class of functions $f$, including the functions $f(\lambda)=\sqrt{\lambda^{2}}$ and $f(\lambda)=\sqrt{\lambda^{2}+m^{2}}$ which are of interest in our context, the operator $D_{f}$ is trace-class and its trace can be computed by integrating the kernel on the diagonal. We now explain the precise statement of this result and its relation to the determinant of the boundary layer operator.

In the following, we will denote by $G_{\mathcal{O}, \lambda} \in \mathcal{D}^{\prime}(X \times X)$ the distributional kernel of the resolvent $R_{\mathcal{O}, \lambda}=\left(-\Delta_{\mathcal{O}}-\lambda^{2}\right)^{-1}$. The kernels $G_{\mathcal{O}_{j}, \lambda}=\breve{R}_{\mathcal{O}_{j}, \lambda}$ and $G_{\text {free, } \lambda}=\breve{R}_{\text {free, } \lambda}$ are defined in an analogous way. By elliptic regularity, these Green's distributions are smooth away from the diagonal $x=y$.

Recall that for $\lambda \in \mathbb{C}$ we have the single-layer potential operator

$$
\mathcal{S}_{\lambda}: C^{\infty}(\partial \mathcal{O}) \rightarrow C^{\infty}(\overline{\mathcal{E}}) \oplus C^{\infty}(\overline{\mathcal{O}}) \subset C^{\infty}\left(\mathbb{R}^{d} \backslash \partial \mathcal{O}\right)
$$

given by

$$
\mathcal{S}_{\lambda} f(x)=\int_{\partial \mathcal{O}} G_{\text {free }, \lambda}(x, y) f(y) \mathrm{d} \sigma(y),
$$

where $\mathrm{d} \sigma$ is the surface measure. Let $\gamma: H^{s+\frac{1}{2}}\left(\mathbb{R}^{d}\right) \rightarrow H^{s}(\partial \mathcal{O})$ be the Sobolev trace operator for $s>0$. Properties of the Sobolev trace operator can be found, for instance, in [45]. One can write the above also as $\mathcal{S}_{\lambda}=G_{\text {free, } \lambda} \circ \gamma^{*}$. Restriction of $\mathcal{S}_{\lambda}$ to the boundary defines an operator

$$
Q_{\lambda} f(x)=\int_{\partial \mathcal{O}} G_{\text {free }, \lambda}(x, y) f(y) \mathrm{d} \sigma(y) .
$$

The operator $Q_{\lambda}$ is known to have the following properties.

Since the boundary $\partial \mathcal{O}$ consists of $N$ connected components $\partial \mathcal{O}_{j}$, we therefore have an orthogonal decomposition $L^{2}(\partial \mathcal{O})=\oplus_{j=1}^{N} L^{2}\left(\partial \mathcal{O}_{j}\right)$. Let $p_{j}$ : 
$L^{2}(\partial \mathcal{O}) \rightarrow L^{2}\left(\partial \mathcal{O}_{j}\right)$ be the corresponding orthogonal projection. Now we have

$$
\begin{aligned}
\tilde{Q}_{\lambda} & =\sum_{j=1}^{N} p_{j} Q_{\lambda} p_{j}, \quad T_{\lambda} \\
& =\sum_{j \neq k}^{N} p_{j} Q_{\lambda} p_{k} \quad \text { and } \quad Q_{\lambda}=\tilde{Q}_{\lambda}+T_{\lambda} .
\end{aligned}
$$

In short, $\tilde{Q}_{\lambda}$ and $T_{\lambda}$ are, respectively, the diagonal and off diagonal part of $Q_{\lambda}$. Now let $\mathfrak{D}_{\nu}$ to be a sector in the upper half plane and it is given by

$$
\mathfrak{D}_{\nu}:=\{z \in \mathbb{C} \mid \nu \leq \arg (z) \leq \pi-\nu\},
$$

where it suffices to consider $0<\epsilon<\frac{\pi}{2}$ for our applications.

The operator $Q_{\lambda}$ is invertible for $\operatorname{Im}(\lambda)>0$. Moreover $Q_{\lambda} \tilde{Q}_{\lambda}^{-1}-1$ is traceclass and the Fredholm determinant $\operatorname{det}\left(Q_{\lambda} \tilde{Q}_{\lambda}^{-1}\right)$ of $Q_{\lambda} \tilde{Q}_{\lambda}^{-1}$ can be used to define a function

$$
\Xi(\lambda)=\log \operatorname{det}\left(Q_{\lambda} \tilde{Q}_{\lambda}^{-1}\right)
$$

which is holomorphic in the upper half space and for some $\delta^{\prime}>0$ satisfies the bound

$$
|\Xi(\lambda)|=O\left(e^{-\delta^{\prime} \operatorname{Im}(z)}\right) .
$$

See Theorem 1.6 of [34] for the above bound in the sector of the form $\{z \mid$ $\operatorname{Im}(z) \geq b|\operatorname{Re}(z)|\}$ for some $\delta>0$.

Assume $0<\theta<\frac{\pi}{2}$ and let $\mathfrak{S}_{\theta}$ be the open sector

$$
\mathfrak{S}_{\theta}=\{z \in \mathbb{C}|z \neq 0,| \arg (z) \mid<\theta\} .
$$

Let $\mathcal{P}_{\theta}$ be the set of functions that are holomorphic and polynomially bounded in $\mathfrak{S}_{\theta}$.

Definition 3.1. The space $\tilde{\mathcal{P}}_{\theta}$ is defined to be the space of functions $f$ such that $f(\lambda)=g\left(\lambda^{2}\right)$ for some $g \in \mathcal{P}_{\theta+\epsilon}$ for some $\epsilon>0$ and there exists $a>0$ such that $f=O\left(|z|^{a}\right)$ if $|z|<1$

We then have the following theorem (see Fig. 2 for the contour).
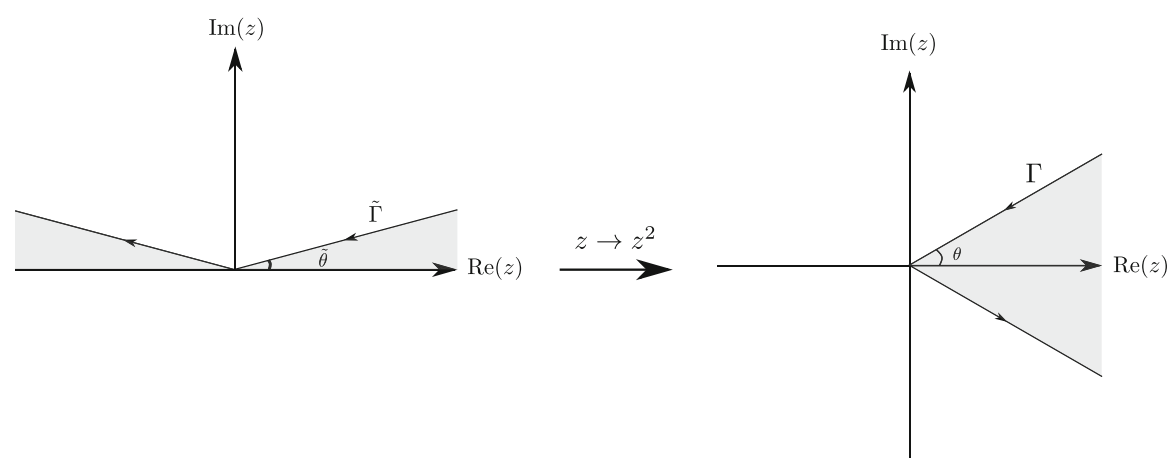

Figure 2. Definition of $\tilde{\Gamma}$ 
Theorem 3.2 ([34], Theorems 1.6 and 1.7). Let $f \in \tilde{\mathcal{P}}_{\theta}$. Then, $D_{f}$ extends to a trace-class operator with integral kernel that is smooth on $X$ and has continuous inner and outer boundary values on $\partial \mathcal{O}$. The trace of $D_{f}$ equals the integral over the diagonal of its integral kernel over $\mathbb{R}^{d}$. Moreover, it is equal to

$$
\operatorname{Tr} D_{f}=\frac{\mathrm{i}}{2 \pi} \int_{\tilde{\Gamma}} f^{\prime}(\lambda) \Xi(\lambda) \mathrm{d} \lambda
$$

In particular, choosing the function $f(\lambda)=\sqrt{\lambda^{2}+m^{2}}-m$ one obtains $D_{f}=H_{\text {rel }}$ and therefore $H_{\text {rel }}$ is trace-class with trace equal to

$$
\operatorname{Tr}\left(H_{\mathrm{rel}}\right)=\frac{1}{\pi} \int_{m}^{\infty} \frac{\omega}{\sqrt{\omega^{2}-m^{2}}} \Xi(\mathrm{i} \omega) \mathrm{d} \omega .
$$

This follows immediately by deforming the contour integral using the exponential decay of $\Xi$ in the upper half plane, considering the branch cut of $\sqrt{z+m^{2}}$ at $\mathrm{i} \sqrt{m}$.

Definition 3.3. The relative stress-energy tensor is the renormalised stressenergy tensor in the relative setting and it is defined as

$$
T_{\text {rel }}=\left(T_{\text {ren }}\right)_{\mathcal{O}}-\sum_{i=1}^{N}\left(T_{\text {ren }}\right)_{\mathcal{O}_{i}},
$$

where $\left(T_{\text {ren }}\right)_{\mathcal{O}}$ is the renormalised stress-energy tensor for obstacle $\mathcal{O}$ and $\left(T_{\text {ren }}\right)_{\mathcal{O}_{i}}$ is the renormalised stress-energy tensor for obstacle $\mathcal{O}_{i}$, which are defined at the beginning of this section.

Remark 3.4. One can also consider other versions of a relative stress-energy tensor. For instance, one can define $\mathcal{O}_{A}=\mathcal{O}_{1} \cup \cdots \cup \mathcal{O}_{j}$ and $\mathcal{O}_{B}=\mathcal{O}_{j+1} \cup \cdots \cup \mathcal{O}_{N}$ for some $1 \leq j<N$, dropping the connectedness requirement of obstacles and work with

$$
T_{\text {rel }, A, B}=\left(T_{\text {ren }}\right)_{\mathcal{O}}-\left(T_{\text {ren }}\right)_{\mathcal{O}_{A}}-\left(T_{\text {ren }}\right)_{\mathcal{O}_{B}}
$$

The corresponding energy encodes the amount of work needed to separate the two obstacle configurations $A$ and $B$. This quantity can also be expressed in terms of $T_{\text {rel }}$ for $A$ and $B$. It is easy to see that this equals

$$
T_{\text {rel }, A, B}=\left(T_{\text {rel }}\right)_{\mathcal{O}}-\left(T_{\text {rel }}\right)_{\mathcal{O}_{A}}-\left(T_{\text {rel }}\right)_{\mathcal{O}_{B}},
$$

and therefore working with $T_{\text {rel }}$ only does not result in a loss of generality.

Theorem 3.5. $T_{\text {rel }}$ is smooth on $X$ and extends smoothly to $\overline{\mathcal{E}}$ as well as to $\overline{\mathcal{O}}$. The function $\left(T_{\mathrm{rel}}\right)_{00}$ is integrable on $\mathbb{R}^{d}$.

Proof. By Theorem 5, we have

$$
\left(T_{\text {rel }}\right)_{00}=\left.\frac{1}{2} \breve{H}_{\text {rel }}\right|_{\Delta}+\frac{1}{8} \Delta\left(\left.\left(\breve{H}^{-1}\right)_{\mathrm{rel}}\right|_{\Delta}\right)
$$

The theorem was shown in [34] for the part $\left.\frac{1}{2} \breve{H}_{\text {rel }}\right|_{\Delta}$ and the same method of proof can also be applied to the second term. We provide the full details here 
for the sake of completeness. We use two estimates proved in [34] which we now explain. Recall that the relative resolvent is given by

$$
R_{\mathrm{rel}, \lambda}:=R_{\mathcal{O}, \lambda}-R_{\text {free }, \lambda}-\sum_{j=1}^{N}\left(R_{\mathcal{O}_{j}, \lambda}-R_{\text {free }, \lambda}\right),
$$

and $R_{\mathcal{O}, \lambda}=\left(-\Delta_{\mathcal{O}}-\lambda^{2}\right)^{-1}, R_{\text {free }, \lambda}=\left(-\Delta_{\text {free }}-\lambda^{2}\right)^{-1}$, and $R_{\mathcal{O}_{i}, \lambda}=\left(-\Delta_{\mathcal{O}_{i}}-\lambda^{2}\right)^{-1}$. For the integral kernel $\breve{R}_{\mathrm{rel}, \lambda}$ we write $G_{\text {rel }, \lambda}$.

As shown in [34] in the proof of Theorem 1.5, the integral kernel $G_{\mathrm{rel}, \lambda}$ of $R_{\text {rel, } \lambda}$ is smooth up to the boundary on $\overline{\mathcal{E}}$ as well as to $\overline{\mathcal{O}}$ and its $C^{k}$-norms on compact subsets $K \subset \overline{\mathcal{E}} \times \overline{\mathcal{E}}$ satisfy the bound

$$
\left\|G_{\mathrm{rel}, \lambda}\right\|_{C^{k}(K)} \leq C_{K, k}(\log |\lambda|)^{\ell} e^{-\delta^{\prime} \operatorname{Im}(\lambda)}
$$

for some $\ell \geq 0$ for $\lambda$ in the sector containing the imaginary axis.

We consider the operator

$$
\left(H^{-1}\right)_{\text {rel }}=H_{\mathcal{O}}^{-1}-H_{\text {free }}^{-1}-\sum_{j=1}^{N}\left(H_{\mathcal{O}_{j}}^{-1}-H_{\text {free }}^{-1}\right)=\frac{2}{\pi} \int_{m}^{\infty} \frac{\lambda}{\sqrt{\lambda^{2}-m^{2}}} R_{\text {rel }, \mathrm{i} \lambda} \mathrm{d} \lambda .
$$

A similar bound holds for $K \subset \overline{\mathcal{O}} \times \overline{\mathcal{E}}, K \subset \overline{\mathcal{E}} \times \overline{\mathcal{O}}$, and $K \subset \overline{\mathcal{O}} \times \overline{\mathcal{O}}$. The log-factor in the estimate is only needed in dimension two. It then follows that $\left(H^{-1}\right)_{\text {rel }}$ has an integral kernel that extends smoothly to $\overline{\mathcal{E}}$ as well as to $\overline{\mathcal{O}}$ (i.e. smooth up to the boundary).

Similarly, $\breve{H}_{\text {rel }}$ is also smooth up to the boundary. Hence, by Theorem 5 and Definition 3.3, we obtain the smoothness of $T_{\text {rel }}$ up to the boundary. In order to show integrability, we recall an estimate for the diagonal of the integral kernel of the resolvent difference, in particular [34]*Theorem 2.9, Equ. (21) and (22).

Let $\lambda \in \mathfrak{D}_{\nu}$ (See (15), i.e. a sector in the upper half plane) and $k_{\mathcal{O}, \lambda}(x, y)$ denote the integral of $R_{\mathcal{O}, \lambda}-R_{\text {free }, \lambda}$, then we have

$$
k_{\mathcal{O}, \lambda}(x, y)=-\left\langle G_{\text {free }, \lambda}(x, \cdot), Q_{\lambda}^{-1}\left(\bar{G}_{\text {free }, \lambda}(y, \cdot)\right)\right\rangle,
$$

which implies

$$
\begin{aligned}
\left|\nabla\left(k_{\mathcal{O}, \lambda}(x, x)\right)\right| \leq & C \sum_{j=1}^{d}\left(\left|\left(\left\langle\partial_{j} G_{\text {free }, \lambda}(x, \cdot), Q_{\lambda}^{-1}\left(\bar{G}_{\text {free }, \lambda}(x, \cdot)\right)\right\rangle\right)\right|\right. \\
& \left.+\left|\left(\left\langle G_{\text {free }, \lambda}(x, \cdot), Q_{\lambda}^{-1}\left(\partial_{j} \bar{G}_{\text {free }, \lambda}(x, \cdot)\right)\right\rangle\right)\right|\right) \\
\leq & C\left|\left(\left\langle\nabla G_{\text {free }, \lambda}(x, \cdot), Q_{\lambda}^{-1}\left(\bar{G}_{\text {free }, \lambda}(x, \cdot)\right)\right\rangle\right)\right|
\end{aligned}
$$

and

$$
\begin{aligned}
\left|\Delta\left(k_{\mathcal{O}, \lambda}(x, x)\right)\right| & \leq C \sum_{j=1}^{d}\left|\partial_{j} \partial_{j}\left(\left\langle G_{\text {free }, \lambda}(x, \cdot), Q_{\lambda}^{-1}\left(\bar{G}_{\text {free }, \lambda}(x, \cdot)\right)\right\rangle\right)\right| \\
& \leq C\left(\left|\left\langle\nabla G_{\text {free }, \lambda}(x, \cdot), Q_{\lambda}^{-1}\left(\nabla \bar{G}_{\text {free }, \lambda}(x, \cdot)\right)\right\rangle\right|\right.
\end{aligned}
$$




$$
\begin{aligned}
& \left.+\left|\left\langle\Delta G_{\text {free }, \lambda}(x, \cdot), Q_{\lambda}^{-1}\left(\bar{G}_{\text {free }, \lambda}(x, \cdot)\right)\right\rangle\right|\right) \\
\leq & C\left(\left\|\nabla G_{\text {free }, \lambda}(x, \cdot)\right\|_{H^{\frac{1}{2}}}\left\|Q_{\lambda}^{-1}\right\|_{H^{\frac{1}{2}} \rightarrow H^{-\frac{1}{2}}}\left\|\nabla \bar{G}_{\text {free }, \lambda}(x, \cdot)\right\|_{H^{\frac{1}{2}}}\right. \\
& \left.\left.+\left\|\Delta G_{\text {free }, \lambda}(x, \cdot)\right\|_{H^{\frac{1}{2}}}\left\|Q_{\lambda}^{-1}\right\|_{H^{\frac{1}{2}} \rightarrow H^{-\frac{1}{2}}} \| \bar{G}_{\text {free }, \lambda}(x, \cdot)\right) \|_{H^{\frac{1}{2}}}\right) .
\end{aligned}
$$

Let $\rho(x)=\operatorname{dist}(x, \partial \mathcal{O})$. By Lemma A.2 of [34] we have for $\rho(x) \lambda \leq 1$,

$$
\sup _{y \in \partial \mathcal{O}}\left|\partial_{x}^{\alpha} \partial_{y}^{\beta} G_{\text {free }, \lambda}(x, y)\right| \leq C \begin{cases}\rho^{-(d-2+|\alpha|+|\beta|)}(x) & (d \geq 3), \\ |\ln (\rho(x) \lambda)|+\rho^{-(|\alpha|+|\beta|)}(x) & (d=2),\end{cases}
$$

and $\ln (\rho(x) \lambda)$ disappears when $|\alpha|+|\beta| \geq 1$. Moreover, for $\rho(x) \lambda>1$, one has

$$
\sup _{y \in \partial \mathcal{O}}\left|\partial_{x}^{\alpha} \partial_{y}^{\beta} G_{\text {free }, \lambda}(x, y)\right| \leq C|\lambda|^{d-2+|\alpha|+|\beta|} e^{\operatorname{Im} \lambda \rho(x)} .
$$

This shows

$$
d \geq 3, \rho(x)>1 \Longrightarrow\left\{\begin{array}{l}
\left\|G_{\text {free }, \lambda}(x, \cdot)\right\|_{H^{\frac{1}{2}}} \leq C_{1} e^{-C_{2}(\operatorname{Im} \lambda) \rho(x)} \rho^{-(d-2)}(x), \\
\left\|\nabla G_{\text {free }, \lambda}(x, \cdot)\right\|_{H^{\frac{1}{2}}} \leq C_{1} e^{-C_{2}(\operatorname{Im} \lambda) \rho(x)} \rho^{-(d-1)}(x), \\
\left\|\Delta G_{\text {free }, \lambda}(x, \cdot)\right\|_{H^{\frac{1}{2}}} \leq C_{1} e^{-C_{2}(\operatorname{Im} \lambda) \rho(x)} \rho^{-d}(x),
\end{array}\right.
$$

and

$$
d=2, \rho(x)>1 \Longrightarrow\left\{\begin{array}{l}
\left\|G_{\text {free }, \lambda}(x, \cdot)\right\|_{H^{\frac{1}{2}}} \leq C_{1} e^{-C_{2}(\operatorname{Im} \lambda) \rho(x)}|\ln (\rho(x) \lambda)|, \\
\left\|\nabla G_{\text {free }, \lambda}(x, \cdot)\right\|_{H^{\frac{1}{2}}} \leq C_{1} e^{-C_{2}(\operatorname{Im} \lambda) \rho(x)} \rho^{-1}(x), \\
\left\|\Delta G_{\text {free }, \lambda}(x, \cdot)\right\|_{H^{\frac{1}{2}}} \leq C_{1} e^{-C_{2}(\operatorname{Im} \lambda) \rho(x)} \rho^{-2}(x),
\end{array}\right.
$$

for some positive $C_{1}$ and $C_{2}$. By Corollary 2.8 of [34] we have

$$
\left\|Q_{\lambda}^{-1}\right\|_{H^{\frac{1}{2}} \rightarrow H^{-\frac{1}{2}}} \leq C\left(1+|\lambda|^{2}\right) .
$$

Now we can conclude that

$$
d \geq 3, \rho(x)>1 \Longrightarrow\left\{\begin{array}{l}
\left|k_{\mathcal{O}, \lambda}(x, x)\right| \leq C_{1} \rho^{-2 d+4}(x) e^{-C_{2} \operatorname{Im} \lambda \rho(x)}, \\
\left|\nabla\left(k_{\mathcal{O}, \lambda}(x, x)\right)\right| \leq C_{1} \rho^{-2 d+3}(x) e^{-C_{2} \operatorname{Im} \lambda \rho(x)} \\
\left|\Delta\left(k_{\mathcal{O}, \lambda}(x, x)\right)\right| \leq C_{1} \rho^{-2(d-1)}(x) e^{-C_{2} \operatorname{Im} \lambda \rho(x)},
\end{array}\right.
$$

and

$$
d=2, \rho(x)>1 \Longrightarrow\left\{\begin{array}{l}
\left|k_{\mathcal{O}, \lambda}(x, x)\right| \leq C_{1} e^{-C_{2}(\operatorname{Im} \lambda) \rho(x)}|\ln (\rho(x) \lambda)|^{2}, \\
\left|\nabla\left(k_{\mathcal{O}, \lambda}(x, x)\right)\right| \leq C_{1} e^{-C_{2}(\operatorname{Im} \lambda) \rho(x)}|\ln (\rho(x) \lambda)| \rho^{-1}(x), \\
\left|\Delta\left(k_{\mathcal{O}, \lambda}(x, x)\right)\right| \leq C_{1} e^{-C_{2} \operatorname{Im} \lambda \rho(x)}|\ln (\rho(x) \lambda)| \rho^{-2}(x) .
\end{array}\right.
$$

Let $h_{\text {rel }}=\left.\left(\breve{H}^{-1}\right)_{\text {rel }}\right|_{\Delta}$. That is $h_{\text {rel }}(x)=\frac{2}{\pi} \int_{m}^{\infty} \frac{\lambda}{\sqrt{\lambda^{2}-m^{2}}} G_{\text {rel }, \mathrm{i} \lambda}(x, x) \mathrm{d} \lambda$. For $m \neq 0$, one can use Eqs. (17) and (18) to get the decay rate of $h_{\text {rel }}(x)$ by integrating over $\lambda$. That is, for $\rho(x)>1, h_{\text {rel }}(x)$ has a decay of $\rho^{-k}(x) e^{-C m \rho(x)}$ with 
both $k$ and $C$ being positive. This warrants the integrability of $\Delta h_{\text {rel }}(x)$ for $d \geq 2, m \neq 0$ and $\rho(x)>1$. Therefore, we will now focus on the case $m=0$. By integrability of the integrand we can interchange differentiation and integration and therefore get

$$
\begin{aligned}
& \nabla h_{\mathrm{rel}}(x)=\nabla\left(\frac{2}{\pi} \int_{0}^{\infty} G_{\mathrm{rel}, \mathrm{i} \lambda}(x, x) \mathrm{d} \lambda\right)=\frac{2}{\pi} \int_{0}^{\infty} \nabla\left(G_{\mathrm{rel}, \mathrm{i} \lambda}(x, x)\right) \mathrm{d} \lambda \\
& \Delta h_{\mathrm{rel}}(x)=\Delta\left(\frac{2}{\pi} \int_{0}^{\infty} G_{\mathrm{rel}, \mathrm{i} \lambda}(x, x) \mathrm{d} \lambda\right)=\frac{2}{\pi} \int_{0}^{\infty} \Delta\left(G_{\mathrm{rel}, \mathrm{i} \lambda}(x, x)\right) \mathrm{d} \lambda .
\end{aligned}
$$

Again, by integrating over $\lambda$, we have

$$
d \geq 2, \rho(x)>1 \Longrightarrow\left\{\begin{array}{l}
\left|\nabla h_{\mathrm{rel}}(x)\right| \leq C \rho^{-2 d+2}(x), \\
\left|\Delta h_{\mathrm{rel}}(x)\right| \leq C \rho^{-2 d+1}(x) .
\end{array}\right.
$$

Let $\Omega \subset \mathbb{R}^{d}$ be an open set with $\operatorname{dist}(\Omega, \partial \mathcal{O})>1$ and $\varphi \in C_{0}^{\infty}\left(\mathbb{R}^{d}\right)$ satisfy $0 \leq \varphi \leq 1$ and $\varphi=1$ in a neighbourhood of $\mathbb{R}^{d} \backslash \Omega$. Then, we have the decomposition

$$
\Delta h_{\mathrm{rel}}=\Delta\left[(1-\varphi) h_{\mathrm{rel}}\right]+\Delta\left(\varphi h_{\mathrm{rel}}\right) .
$$

The integrability of $\Delta\left(\varphi h_{\mathrm{rel}}\right)$ in Eq. (20) follows from the smoothness property of the kernel of $\left(\breve{H}^{-1}\right)_{\text {rel }}$ at the diagonal as shown above. Thereby the integrability of $\Delta h_{\text {rel }}$ on $\mathbb{R}^{d}$ is equivalent to the one of $\Delta\left[(1-\varphi) h_{\text {rel }}\right]$ on $\operatorname{supp}(1-\varphi)$. This follows immediately from Eq. (19). Therefore, we have shown the integrability of $\Delta\left(\left.\left(\breve{H}^{-1}\right)_{\text {rel }}\right|_{\Delta}\right)$ on $\mathbb{R}^{d}$. Finally, the integrability of $T_{\text {rel }}$ follows from Theorem 2.4 and the definition of relative stress-energy tensor (i.e. Definition $3.3)$.

Definition 3.6. The relative energy is defined as

$$
E_{\text {rel }}=\int_{\mathbb{R}^{d}}\left(T_{\text {rel }}\right)_{00} \mathrm{~d} x=\int_{\mathbb{R}^{d}}\left(\left(\left(T_{\text {ren }}\right)_{\mathcal{O}}\right)_{00}-\sum_{i=1}^{N}\left(\left(T_{\text {ren }}\right)_{\mathcal{O}_{i}}\right)_{00}\right) \mathrm{d} x .
$$

Theorem 3.7. We have the equality

$$
E_{\mathrm{rel}}=\frac{1}{2} \operatorname{Tr} H_{\mathrm{rel}}=\frac{1}{\pi} \int_{m}^{\infty} \frac{\omega}{\sqrt{\omega^{2}-m^{2}}} \Xi(\mathrm{i} \omega) \mathrm{d} \omega .
$$

Proof. We have

$$
\int_{\mathbb{R}^{d}} \Delta\left(\left.\left(\breve{H}^{-1}\right)_{\operatorname{rel}}\right|_{\Delta}\right) \mathrm{d} x=\lim _{r \rightarrow \infty} \int_{B_{r}} \Delta\left(\left.\left(\breve{H}^{-1}\right)_{\operatorname{rel}}\right|_{\Delta}\right) \mathrm{d} x,
$$

where $B_{r}$ is the ball of radius $r$ centred at the origin. As $\Delta\left(\left.\left(\breve{H}^{-1}\right)_{\text {rel }}\right|_{\Delta}\right)$ has only jump-type discontinuity across $\partial X$, we can apply the divergence theorem to the integral on $B_{r}$ for sufficiently large $r$. That is

$$
\begin{aligned}
\int_{B_{r}} \Delta\left(\left.\left(\breve{H}^{-1}\right)_{\operatorname{rel}}\right|_{\Delta}\right) \mathrm{d} x= & \int_{\partial B_{r}} \partial_{\nu}\left(\left.\left(\breve{H}^{-1}\right)_{\operatorname{rel}}\right|_{\Delta}\right) \mathrm{d} \sigma(x) \\
& +\int_{\partial X}\left(\partial_{\nu}\left(\left.\left(\breve{H}^{-1}\right)_{\operatorname{rel}}\right|_{\Delta}\right)\right)_{+} \mathrm{d} \sigma(x) \\
& +\int_{\partial X}\left(\partial_{\nu}\left(\left.\left(\breve{H}^{-1}\right)_{\mathrm{rel}}\right|_{\Delta}\right)\right)_{-} \mathrm{d} \sigma(x)
\end{aligned}
$$


where $(\cdot)_{+}$and $(\cdot)_{-}$are the exterior and interior limits, respectively. From equation (19), we also have

$$
\left|\nabla\left[(1-\varphi) h_{\mathrm{rel}}\right]\right|(x) \leq \frac{C_{\Omega}}{(\operatorname{dist}(x, \partial \mathcal{O}))^{2 d-2}},
$$

which implies the contribution of the integral over $\partial B_{r}$ vanished as $r \rightarrow \infty$ and therefore

$$
\begin{aligned}
\int_{\mathbb{R}^{d}} \Delta\left(\left.\left(\breve{H}^{-1}\right)_{\operatorname{rel}}\right|_{\Delta}\right) \mathrm{d} x=\int_{\partial X} & \left(\partial_{\nu}\left(\left.\left(\breve{H}^{-1}\right)_{\text {rel }}\right|_{\Delta}\right)\right)_{+} \mathrm{d} \sigma(x) \\
& +\int_{\partial X}\left(\partial_{\nu}\left(\left.\left(\breve{H}^{-1}\right)_{\mathrm{rel}}\right|_{\Delta}\right)\right)_{-} \mathrm{d} \sigma(x) .
\end{aligned}
$$

From Eq. (16), we then have

$$
\begin{aligned}
\int_{\mathbb{R}^{d}} \nabla \cdot \nabla\left(\left.\left(\breve{H}^{-1}\right)_{\mathrm{rel}}\right|_{\Delta}\right) \mathrm{d} x & =\int_{\mathbb{R}^{d}} \Delta\left(\left.\left(\breve{H}^{-1}\right)_{\mathrm{rel}}\right|_{\Delta}\right) \mathrm{d} x \\
& =\frac{2}{\pi} \int_{\mathbb{R}^{d}} \int_{0}^{\infty}\left[\nabla \cdot \nabla\left(\left.G_{\mathrm{rel}, \mathrm{i} \lambda}\right|_{\Delta}\right)\right] \mathrm{d} \lambda \mathrm{d} x \\
& =\frac{2}{\pi} \int_{\mathbb{R}^{d}} \int_{0}^{\infty}\left[2 \nabla \cdot\left(\left.\nabla G_{\mathrm{rel}, \mathrm{i} \lambda}\right|_{\Delta}\right)\right] \mathrm{d} \lambda \mathrm{d} x \\
& =2 \int_{\mathbb{R}^{d}}\left[\nabla \cdot\left(\left.\nabla\left(\breve{H}^{-1}\right)_{\mathrm{rel}}\right|_{\Delta}\right)\right] \mathrm{d} x .
\end{aligned}
$$

This shows

$$
\begin{aligned}
\frac{1}{2} \int_{\mathbb{R}^{d}} \Delta\left(\left.\left(\breve{H}^{-1}\right)_{\mathrm{rel}}\right|_{\Delta}\right) \mathrm{d} x= & \int_{\partial X}\left(\left.\partial_{\nu}\left(\breve{H}^{-1}\right)_{\mathrm{rel}}\right|_{\Delta}\right)_{+} \mathrm{d} \sigma(x) \\
& +\int_{\partial X}\left(\left.\partial_{\nu}\left(\breve{H}^{-1}\right)_{\mathrm{rel}}\right|_{\Delta}\right)_{-} \mathrm{d} \sigma(x) .
\end{aligned}
$$

We start by showing that for the restrictions to $\partial \mathcal{O}_{q}$ we have the following identity

$$
\left[\left.\partial_{\nu}\left(\breve{H}_{\mathcal{O}}^{-1}-\breve{H}_{\text {free }}^{-1}-\left(\breve{H}_{\mathcal{O}_{q}}^{-1}-\breve{H}_{\text {free }}^{-1}\right)\right)\right|_{\Delta}\right]_{\partial \mathcal{O}_{q}, \pm}=\left[\left.\partial_{\nu}\left(\breve{H}_{\mathcal{O}}^{-1}-\breve{H}_{\mathcal{O}_{q}}^{-1}\right)\right|_{\Delta}\right]_{\partial \mathcal{O}_{q}, \pm}=0
$$

To see this, we temporarily denote by $k(x, y)$ the integral kernel of $\left(H_{\mathcal{O}}^{-1}-H_{\mathcal{O}_{q}}^{-1}\right)$. This kernel vanishes $\mathcal{O}_{q} \times \mathcal{O}_{q}$ and the interior normal derivative therefore vanishes trivially. We therefore only need to concern ourself with the exterior normal derivative. As shown in the proof of Theorem 3.5 the kernel $\left(H^{-1}\right)_{\text {rel }}$ is smooth. One concludes from this, using Theorem 2.2 , that $\left(H_{\mathcal{O}}^{-1}-H_{\mathcal{O}_{q}}^{-1}\right)$ is smooth near $\mathcal{O}_{q} \times \mathcal{O}_{q}$ in $\overline{\mathcal{E}} \times \overline{\mathcal{E}}$. The kernel $k$ satisfies Dirichlet boundary conditions in both variables in the sense that $k(x, y)=0$ if $y \in \partial \mathcal{O}_{q}$ or if $x \in \partial \mathcal{O}_{q}$. By the chain rule $\left[\left.\partial_{\nu}\left(\breve{H}_{\mathcal{O}}^{-1}-\breve{H}_{\mathcal{O}_{q}}^{-1}\right)\right|_{\Delta}\right]_{ \pm}(x)$ equals $\left.\left(\partial_{\nu, x} k(x, y)+\partial_{\nu, y} k(x, y)\right)\right|_{y=x}$, 
which therefore vanishes on $\partial \mathcal{O}_{q}$. We then have

$$
\begin{aligned}
& \int_{\partial X}\left(\left.\partial_{\nu}\left(\breve{H}^{-1}\right)_{\text {rel }}\right|_{\Delta}\right)_{ \pm} \mathrm{d} \sigma(x) \\
& =\sum_{q=1}^{N} \int_{\partial \mathcal{O}_{q}}\left(\left.\partial_{\nu}\left(\breve{H}^{-1}\right)_{\text {rel }}\right|_{\Delta}\right)_{ \pm} \mathrm{d} \sigma(x) \\
& =\sum_{q=1}^{N} \int_{\partial \mathcal{O}_{q}}\left[\left.\partial_{\nu}\left(\breve{H}_{\mathcal{O}}^{-1}-\breve{H}_{\text {free }}^{-1}-\sum_{p=1}^{N}\left(\breve{H}_{\mathcal{O}_{p}}^{-1}-\breve{H}_{\text {free }}^{-1}\right)\right)\right|_{\Delta}\right]_{ \pm} \mathrm{d} \sigma(x) \\
& =\sum_{q=1}^{N} \int_{\partial \mathcal{O}_{q}}\left[-\left.\sum_{p \neq q}^{N} \partial_{\nu}\left(\breve{H}_{\mathcal{O}_{p}}^{-1}-\breve{H}_{\text {free }}^{-1}\right)\right|_{\Delta}\right]_{ \pm} \mathrm{d} \sigma(x) .
\end{aligned}
$$

By Theorem 2.2, we know that $\left.\partial_{\nu}\left(\breve{H}_{\mathcal{O}_{p}}^{-1}-\breve{H}_{\text {free }}^{-1}\right)\right|_{\Delta}$ is smooth across the boundary $\partial \mathcal{O}_{q}$ for $p \neq q$, which implies

$$
\int_{\partial \mathcal{O}_{q}}\left[-\left.\sum_{p \neq q}^{N} \partial_{\nu}\left(\breve{H}_{\mathcal{O}_{p}}^{-1}-\breve{H}_{\text {free }}^{-1}\right)\right|_{\Delta}\right]_{+} \mathrm{d} \sigma(x)=-\int_{\partial \mathcal{O}_{q}}\left[-\left.\sum_{p \neq q}^{N} \partial_{\nu}\left(\breve{H}_{\mathcal{O}_{p}}^{-1}-\breve{H}_{\text {free }}^{-1}\right)\right|_{\Delta}\right]_{-} \mathrm{d} \sigma(x) .
$$

Hence, we have $\int_{\mathbb{R}^{d}} \Delta\left(\left.\left(\breve{H}^{-1}\right)_{\mathrm{rel}}\right|_{\Delta}\right) \mathrm{d} x=0$, which verifies the first Eq. in $(21)$. The representation of the trace of $H_{\text {rel }}$ in terms of $\Xi$ follows via $\Xi^{\prime}(\lambda)=$ $-2 \lambda \operatorname{Tr}\left(R_{\mathrm{rel}, \lambda}\right)$ from Theorem 4.2 in [34], Theorem 3.2 and equation (16).

\section{Estimates on the Relative Resolvent}

In preparation for the proof of the variational formula, we will need some additional estimates on the relative resolvent $R_{\mathrm{rel}, \lambda}$, which we collect in this section. We have the following well-known layer potential representation (see for example [34]*Equ. (19))

$$
R_{\mathcal{O}, \lambda}-R_{\text {free }, \lambda}=-\mathcal{S}_{\lambda} Q_{\lambda}^{-1} \mathcal{S}_{\lambda}^{t},
$$

which gives

$$
R_{\mathrm{rel}, \lambda}=-\mathcal{S}_{\lambda}\left(Q_{\lambda}^{-1}-\tilde{Q}_{\lambda}^{-1}\right) \mathcal{S}_{\lambda}^{t}
$$

Also, let $\rho$ be the function defined as

$$
\rho(\lambda):= \begin{cases}\lambda^{d-4} & \text { if } 0<\lambda \leq 1 \text { and } d=2,3 \\ |\log \lambda|+1 & \text { if } 0<\lambda \leq 1 \text { and } d=4, \\ 1 & \text { if } 0<\lambda \leq 1 \text { and } d>4 \\ 1 & \text { if } \lambda>1 .\end{cases}
$$

For $i, j, k \in \mathbb{Z}$, let $\rho_{i, j ; k}$ be the functions defined as

$$
\rho_{i, j ; k}(\lambda):= \begin{cases}(|\log \lambda|+1)^{i} \lambda^{j} & \text { for } 0<\lambda \leq 1 \\ \lambda^{k} & \text { for } \lambda>1\end{cases}
$$

The following proposition partially follows from [34, Proposition 2.1] and extends [34, Proposition 2.2]. Let us summarise some mapping properties of the layer potential operators. 
Proposition 4.1. Let $d \geq 2$ and $-\frac{3}{2} \leq s \leq-\frac{1}{2}$. Then, we have the following properties of $\mathcal{S}_{\lambda}$ for $\lambda \in \mathfrak{D}_{\nu}$ (See (15), i.e. a sector in the upper half plane).

(1) If $\operatorname{Re}(\lambda) \neq 0$ then $\left\|\mathcal{S}_{\lambda}\right\|_{H^{s}(\partial \mathcal{O}) \rightarrow L^{2}\left(\mathbb{R}^{d}\right)} \leq C_{s} \frac{\sqrt{1+|\lambda|^{2}}}{\operatorname{Re}(\lambda) \operatorname{Im}(\lambda)}$.

(2) Let $\chi \in C^{\infty}\left(\mathbb{R}^{d}\right)$ be supported in $V$, where $V \subset \mathbb{R}^{d}$ is an open set with smooth boundary and $\operatorname{dist}(V, \partial \mathcal{O})>0$. For $0<\delta<\operatorname{dist}(V, \partial \mathcal{O})$, we have that $\chi \mathcal{S}_{\lambda}: H^{s}(\partial \mathcal{O}) \rightarrow L^{2}(V)$ is a Hilbert-Schmidt operator whose HilbertSchmidt norm is bounded by

$$
\left\|\chi \mathcal{S}_{\lambda}\right\|_{H S\left(H^{s}(\partial \mathcal{O}) \rightarrow L^{2}\left(\mathbb{R}^{d}\right)\right)} \leq C_{\delta, \nu, k} \sqrt{\rho(\operatorname{Im} \lambda)} e^{-\delta \operatorname{Im} \lambda}
$$

which implies

$$
\left\|\mathcal{S}_{\lambda}\right\|_{H^{s}(\partial \mathcal{O}) \rightarrow L^{2}\left(\mathbb{R}^{d}\right)} \leq C_{\delta, \nu, s}(1+\sqrt{\rho(\operatorname{Im} \lambda)}) .
$$

(3) $\left\|Q_{\lambda}^{-1}\right\|_{H^{\frac{1}{2}}(\partial \mathcal{O}) \rightarrow H^{-\frac{1}{2}}(\partial \mathcal{O})} \leq C_{\nu}\left(1+(\operatorname{Im} \lambda)^{2}\right)^{2}$.

Proof. Recall that we could also write the single-layer potential operator as $\mathcal{S}_{\lambda}=G_{\text {free, } \lambda} \circ \gamma^{*}$. Note that $\gamma^{*}: H^{s}(\partial \mathcal{O}) \rightarrow H^{s-\frac{1}{2}}\left(\mathbb{R}^{d}\right), G_{\text {free }, \lambda}: H^{s-\frac{1}{2}}\left(\mathbb{R}^{d}\right) \rightarrow$ $H^{s+\frac{3}{2}}\left(\mathbb{R}^{d}\right)$ and the natural inclusion map $\iota: H^{s+\frac{3}{2}}\left(\mathbb{R}^{d}\right) \rightarrow L^{2}\left(\mathbb{R}^{d}\right)$ are bounded maps. For $-\frac{3}{2} \leq s \leq-\frac{1}{2}$, their norms are bounded by

$$
\left\{\begin{array}{l}
\left\|\gamma^{*}\right\|_{H^{s} \rightarrow H^{s-\frac{1}{2}}} \leq C_{s}, \\
\left\|G_{\text {free }, \lambda}\right\|_{H^{s-\frac{1}{2}} \rightarrow H^{s+\frac{3}{2}}} \leq \sup _{x \in \mathbb{R}}\left|\frac{\sqrt{1+x^{2}}}{x^{2}-\lambda^{2}}\right| \leq \frac{\sqrt{1+|\lambda|^{2}}}{\operatorname{Re}(\lambda) \operatorname{Im}(\lambda)}, \\
\|\iota\|_{H^{s+\frac{3}{2}} \rightarrow H^{0}} \leq 1 .
\end{array}\right.
$$

The second bound follows from the spectral representation of $-\Delta_{\text {free }}$. Therefore, we conclude the proof for part (1).

Part (3) follows immediately from the bound on the Dirichlet-to-Neumann operator in [34]. For part (2), let $\mathcal{O} \subset V^{\prime} \subset \mathbb{R}^{d}$ be an open set with $\operatorname{dist}\left(V, V^{\prime}\right)>$ 0 and choose $0<\delta<\operatorname{dist}\left(V, V^{\prime}\right)$. In particular, $0<\delta<\operatorname{dist}(V, \partial \mathcal{O})$. Let $k \in \mathbb{N}$. Now we have from [34] the estimate

$$
\begin{aligned}
& \left\|(-\Delta)^{\frac{k}{2}} G_{\text {free }, \lambda}\right\|_{L^{2}\left(V \times V^{\prime}\right)}^{2} \\
& \quad \leq C_{\delta, \nu, k} \begin{cases}(\operatorname{Im} \lambda)^{2 k-2}\left(1+(\operatorname{Im} \lambda \log (\operatorname{Im} \lambda))^{2}\right) e^{-2 \delta \operatorname{Im} \lambda} & \text { for } d=2 \\
(\operatorname{Im} \lambda)^{2 k}(1+\log (\operatorname{Im} \lambda)) e^{-2 \delta \operatorname{Im} \lambda} & \text { for } d=4 \\
(\operatorname{Im} \lambda)^{d+2 k-4}\left(1+|\operatorname{Im} \lambda|^{4-d}\right) e^{-2 \delta \operatorname{Im} \lambda} & \text { for } d \neq 2,4\end{cases}
\end{aligned}
$$

where $\Delta=\Delta_{x}+\Delta_{y}$ is the Laplace operator on $V \times V^{\prime}$. In other words, we have

$$
\left\|G_{\text {free }, \lambda}\right\|_{H^{k}\left(V \times V^{\prime}\right)}^{2} \leq C_{\delta, \nu, k}\left(1+(\operatorname{Im} \lambda)^{2 k}\right) \rho(\operatorname{Im} \lambda) e^{-2 \delta \operatorname{Im} \lambda} .
$$

Now by taking Sobolev trace, we have

$$
\left\|G_{\text {free }, \lambda}\right\|_{H^{s}(V \times \partial \mathcal{O})}^{2} \leq C_{\delta, \nu, k}\left(1+(\operatorname{Im} \lambda)^{2 s+1}\right) \rho(\operatorname{Im} \lambda) e^{-2 \delta \operatorname{Im} \lambda} .
$$


Statement (2) follows, using the properties of the Hilbert-Schmidt norm (for example Sect. A.3.1 in [43]). Since $-\frac{3}{2} \leq s \leq-\frac{1}{2}$, we have

$$
\begin{aligned}
\left\|\chi \mathcal{S}_{\lambda}\right\|_{H S\left(H^{s}(\partial \mathcal{O}) \rightarrow L^{2}(V)\right)}^{2} & \leq C_{1}\left\|\chi \mathcal{S}_{\lambda} E_{-s}\right\|_{H S\left(L^{2}(\partial \mathcal{O}) \rightarrow L^{2}(V)\right)}^{2} \\
& \leq C_{2}\left\|G_{\text {free, }, \lambda}\right\|_{H^{-s}}^{2}(V \times \partial \mathcal{O}) \\
& \leq C_{\delta, \nu, k}\left(1+(\operatorname{Im} \lambda)^{-2 s+1}\right) \rho(\operatorname{Im} \lambda) e^{-2 \delta \operatorname{Im} \lambda} \\
& \leq C_{\delta, \nu, k} \rho(\operatorname{Im} \lambda) e^{-2 \delta \operatorname{Im} \lambda}
\end{aligned}
$$

where $E_{s}=\left(\sqrt{-\Delta_{\partial \mathcal{O}}}+1\right)^{s}$. To prove the last property of $\mathcal{S}_{\lambda}$, we are left with proving the bound for $\phi \mathcal{S}_{\lambda}$, where $\phi \in C^{\infty}\left(V^{\prime \prime}\right)$ is supported in $V^{\prime \prime}$ with $\mathcal{O} \subset$ $V^{\prime} \subset V^{\prime \prime}, \phi(x)=1$ on $\mathcal{O}$ and $1-\phi$ is supported in $V$. It suffices to bound $\phi G_{\text {free }, \lambda} \phi: H^{s}\left(\mathbb{R}^{d}\right) \rightarrow L^{2}\left(\mathbb{R}^{d}\right)$ for $-\frac{3}{2} \leq s \leq-\frac{1}{2}$. Note that the explicit kernel of $\phi G_{\text {free }, \lambda} \phi$, denoted by $K_{\lambda}(x, y)$, is given by

$$
K_{\lambda}(x, y)=\frac{i}{4} \phi(x) \phi(y)\left(\frac{\lambda}{2 \pi|x-y|}\right)^{\frac{d-2}{2}} H_{\frac{d-2}{2}}^{(1)}(\lambda|x-y|),
$$

where $H_{\nu}^{(1)}$ is the Hankel function. By the Schur test and estimates on the free resolvent (see Appendix A for details), we have

$$
\left\|\phi G_{\text {free }, \lambda} \phi\right\|_{L^{2} \rightarrow H^{2}} \leq C_{r_{0}, \phi} \begin{cases}\rho_{1,0 ; 0}(\operatorname{Im} \lambda) & \text { for } d=2 \\ 1 & \text { for } d \neq 2 .\end{cases}
$$

Taking the adjoint, we get the same bound for $H^{-2} \rightarrow L^{2}$. Finally, using the estimate (24) with $\chi=1-\phi$, we obtain

$$
\left\|\mathcal{S}_{\lambda}\right\|_{H^{s}(\partial \mathcal{O}) \rightarrow L^{2}\left(\mathbb{R}^{d}\right)} \leq C_{\delta, \nu, s}(1+\sqrt{\rho(\operatorname{Im} \lambda)}) \quad \text { for }-\frac{3}{2} \leq s \leq-\frac{1}{2} .
$$

Remark 4.2. In the case of odd dimensions, an easier argument can be used to provide a weaker estimate that is still sufficient for the purposes of this paper. Using the strong Huygens principle, one deduces ( [47, Sect. 3.1])

$$
\left\|\phi G_{\text {free }, \lambda} \phi\right\|_{H^{-2} \rightarrow L^{2}} \leq C_{\phi}(1+|\lambda|) \text { for } \quad \lambda \in \mathfrak{D}_{\nu},
$$

which implies

$$
\left\|\phi G_{\text {free }, \lambda} \phi\right\|_{H^{s-\frac{1}{2}} \rightarrow L^{2}} \leq C_{\phi}(1+|\lambda|) \leq C_{\phi, \nu}(1+\operatorname{Im} \lambda) \quad \text { for } \quad-\frac{3}{2} \leq s \leq-\frac{1}{2} .
$$

This gives $\left\|\phi \mathcal{S}_{\lambda}\right\|_{H^{s} \rightarrow L^{2}} \leq C_{\phi, \nu}(1+\operatorname{Im} \lambda)$ for $-\frac{3}{2} \leq s \leq-\frac{1}{2}$. Applying inequality (24) for $\chi=1-\phi$ and using $\frac{-2 s+1}{2} \geq 1$, we get

$$
\left\|\mathcal{S}_{\lambda}\right\|_{H^{s} \rightarrow L^{2}} \leq C_{\delta, \nu, s}(1+\operatorname{Im} \lambda+\sqrt{\rho(\operatorname{Im} \lambda)}) \quad \text { for }-\frac{3}{2} \leq s \leq-\frac{1}{2} .
$$




\section{Hadamard Variation Formula and Equivalence of Approaches (2) and (3)}

In this section, we will show that a version of Hadamard variation of the renormalised stress-energy tensor $\left(T_{\text {ren }}\right)_{i j}$ defined in Sect. 2.2 is related to the Hadamard variation formula for the resolvent. We will follow the methods developed in $[39,41,48,49]$ and derive a Hadamard variation formula for the relative resolvent, then apply it to the relative stress-energy tensor. Short proofs of Hadamard variation formula can be found in $[50,51]$ for the case of bounded domains. Since we are dealing with an unbounded domain, we extend theory to non-compact setting for the special case of boundary translation flows (see Definition 5.1) in Theorem 5.3, which are sufficient for our purposes.

\subsection{Hadamard Variation Formula}

Let $U$ be a possibly unbounded open subset in $\mathbb{R}^{d}$ with smooth compact boundary and $Y$ be a smooth vector field on $\mathbb{R}^{d}$. The flow, denoted by $\varphi_{\epsilon}$ and generated by $Y$, gives, for small $|\epsilon|$, a one-parameter family of smooth manifolds in $\mathbb{R}^{d}$, which is denoted by $U_{\epsilon}=\varphi_{\epsilon}(U)$. For our application, $U$ would be either $\mathcal{E}$ or $\mathcal{O}$ as defined in Sect. 1 and we will only consider flows that generate rigid translations of obstacles.

Definition 5.1. A flow $\varphi_{\epsilon}$ associated with vector field $Y$ is called a boundary translation if $Y$ is locally constant near $\partial U$.

Following Peetre's derivation of Hadamard variation formula, we define the following variational derivative.

Definition 5.2. Let $u_{\epsilon}$ be a (weak-*) $C^{1}$ curve of functions in $\mathcal{D}^{\prime}\left(U_{\epsilon}\right)$. The variational derivative at $\epsilon=0$ is given by

$$
\delta_{Y} u:=\theta_{Y} u-Y u,
$$

where $\theta_{Y} u=\lim _{\epsilon \rightarrow 0} \frac{\varphi_{\epsilon}^{*} u_{\epsilon}-u_{0}}{\epsilon}$ is the variational derivative defined by GarabedianSchiffer's in [39]. Here, the derivative $\theta_{Y} u$ is understood in the weak- $\star$-sense and the action of the vector field $Y$ is understood in the sense of distribution.

Note that $\theta_{Y} u$ is different from the standard (conventional) Lie derivative, as $u_{\epsilon}$ may have an additional dependence on $\epsilon$. In fact, the last term, $Y u$ in Eq. (26), should be understood as the conventional Lie derivative of $u_{0}$.

The derivation of Hadamard variational formula for the resolvent associated with the Dirichlet Laplace operator usually starts with the energy quadratic form (see $[39,41,49]$ ). The energy quadratic form associated with the Dirichlet Laplacian $-\Delta_{U_{\epsilon}}$ on $U_{\epsilon}$ is given by

$$
E_{\epsilon}(u, v ; \lambda)=\int_{U_{\epsilon}}\left(\nabla u \cdot \overline{\nabla v}-\lambda^{2} u \bar{v}\right) \mathrm{d} x,
$$

where $\lambda \in \mathbb{C}$ and $\operatorname{Im} \lambda>0$. Using the diffeomorphism flow $\varphi_{\epsilon}$, one can pull-back the quadratic form from $U_{\epsilon}$ to $U_{0}=U$, which gives a one-parameter family of quadratic forms on $U$, i.e.

$$
\tilde{E}_{\epsilon}(u, v ; \lambda)=\int_{U}\left[\varphi_{\epsilon}^{*}\left(\nabla \varphi_{\epsilon}^{-1 *}(u) \cdot \overline{\nabla \varphi_{\epsilon}^{-1 *}(v)}\right)-\lambda^{2} u \bar{v}\right] \varphi_{\epsilon}^{*}(\mathrm{~d} x) .
$$


Note that the energy form (27) and the induced energy forms (28) are related by

$$
E_{\epsilon}(u, v ; \lambda)=\tilde{E}_{\epsilon}\left(\varphi_{\epsilon}^{*}(u), \varphi_{\epsilon}^{*}(v) ; \lambda\right)
$$

The operator associated with the energy form (27) is the Dirichlet Laplace operator, whereas $\tilde{E}_{\epsilon}$ defines a one-parameter families of elliptic operators on $U$ for sufficiently small $\epsilon$. Let $G_{\lambda, \epsilon}=G_{U_{\epsilon}, \lambda}$ be kernel of the resolvent for the Dirichlet Laplacian on $U_{\epsilon}$. Then, from Eqs. (27), (28) and (29), we have in the sense of distributions

$$
\left\{\begin{array}{l}
\left(-\Delta_{x}-\lambda^{2}\right) G_{\lambda, \epsilon}(x, y)=\delta_{y}(x) \text { in } U_{\epsilon} \\
G_{\lambda, \epsilon}(x, y)=0 \text { for } \quad x \in \partial U_{\epsilon}
\end{array},\right.
$$

where $y$ is in the interior of $U_{\epsilon}$ and by elliptic regularity $G_{\lambda, \epsilon}(x, y)$ is then smooth at the boundary and therefore it makes sense to define its boundary value.

As we would like to study the variation of resolvents, it is convenient to consider the variation as distributions on $U \times U$. In other words, for $R_{\epsilon} \epsilon$ $\mathcal{D}^{\prime}\left(U_{\epsilon} \times U_{\epsilon}\right)$ and $u, v \in C_{0}^{\infty}(U)$, we have, from the Schwartz kernel theorem,

$$
\varphi_{\epsilon}^{*} R_{\epsilon}(u \otimes v)=R_{\epsilon}\left(\varphi_{-\epsilon}^{*} u \otimes \varphi_{-\epsilon}^{*} v\right)=\left\langle\varphi_{-\epsilon}^{*} u, R_{\epsilon} \varphi_{-\epsilon}^{*} \bar{v}\right\rangle=\left\langle u, \varphi_{\epsilon}^{*} R_{\epsilon} \varphi_{-\epsilon}^{*} \bar{v}\right\rangle,
$$

where the first two brackets correspond to the pairing between distributions and test functions while the third and the forth brackets are the $L^{2}$ inner products on $U_{\epsilon}$ and $U$, respectively. It is not hard to see that the existence of the variational derivative of $R_{\epsilon}$ in the sense of (26) is implied by the existence of $\theta_{Y} R$. From Eq. (31), the existence of $\theta_{Y} R=\lim _{\epsilon \rightarrow 0} \frac{\varphi_{\epsilon}^{*} R_{\epsilon}-R_{0}}{\epsilon}$ in the weak-*-sense is equivalent to the existence of the standard derivative of $r(\epsilon)=\left\langle u, \varphi_{\epsilon}^{*} R_{\epsilon} \varphi_{-\epsilon}^{*} v\right\rangle$ with respect to $\epsilon$ for all $u, v \in C_{0}^{\infty}(U)$.

The following theorem is the well-known Hadamard variation formula for the resolvent extended to case of unbounded domains in our setting.

Theorem 5.3. Let $\varphi_{\epsilon}$ be a boundary translation flow, then the variational derivative of $R_{\lambda, \epsilon}$ exists in the weak-* topology. Let $G_{\lambda, \epsilon}(x, y)$ be the kernel of $R_{\lambda, \epsilon}$, then its variational derivative is given by

$$
\delta_{Y} G_{\lambda, 0}(x, y)=\int_{\partial U} \partial_{\nu}^{\prime} G_{\lambda, 0}(x, z) \partial_{\nu} G_{\lambda, 0}(z, y)\langle Y, \nu\rangle \mathrm{d} \sigma(z) .
$$

Proof. Firstly, we prove the existence of $\theta_{Y} R_{\lambda}$ in weak-*-sense. We know that, from Eq. (23), $\varphi_{\epsilon}^{*} R_{\lambda, \epsilon} \varphi_{-\epsilon}^{*}-R_{\text {free }, \lambda}=-\varphi_{\epsilon}^{*} \mathcal{S}_{\lambda, \epsilon} Q_{\lambda, \epsilon}^{-1} \mathcal{S}_{\lambda, \epsilon}^{t} \varphi_{-\epsilon}^{*}$. We will therefore establish differentiability of

$$
-\left\langle g, \varphi_{\epsilon}^{*} \mathcal{S}_{\lambda, \epsilon} Q_{\lambda, \epsilon}^{-1} \mathcal{S}_{\lambda, \epsilon}^{t} \varphi_{-\epsilon}^{*} f\right\rangle=-\left\langle g,\left(\varphi_{\epsilon}^{*} \mathcal{S}_{\lambda, \epsilon} \varphi_{-\epsilon}^{*}\right)\left(\varphi_{\epsilon}^{*} Q_{\lambda, \epsilon}^{-1} \varphi_{-\epsilon}^{*}\right)\left(\varphi_{\epsilon}^{*} \mathcal{S}_{\lambda, \epsilon}^{t} \varphi_{-\epsilon}^{*}\right) f\right\rangle
$$

for any fixed test functions $g, f \in C_{0}^{\infty}(U)$ and compute its derivative. In the last term of Eq. (33), the operator $\mathcal{S}_{\lambda, \epsilon}^{t}$ is the transpose operator to $\mathcal{S}_{\lambda, \epsilon}$ obtained from the real inner product, i.e. $\mathcal{S}_{\lambda, \epsilon}^{t} f=\overline{\mathcal{S}_{\lambda, \epsilon}^{t} \bar{f}}$. Since the free resolvent is smooth 
off the diagonal and

$$
\begin{aligned}
\left\langle g, \varphi_{\epsilon}^{*} \mathcal{S}_{\lambda, \epsilon} \varphi_{-\epsilon}^{*} f\right\rangle & =\int_{U} \int_{\partial U_{\epsilon}} g(x) G_{\text {free }, \lambda}\left(\varphi_{\epsilon}(x), \tilde{y}\right) f\left(\varphi_{-\epsilon}(\tilde{y})\right) \mathrm{d} \sigma(\tilde{y}) \mathrm{d} x \\
& =\int_{U} \int_{\partial U} g(x) G_{\text {free }, \lambda}\left(\varphi_{\epsilon}(x), \varphi_{\epsilon}(y)\right) f(y) \mathrm{d} \sigma(y) \mathrm{d} x
\end{aligned}
$$

the kernel of $\mathcal{S}_{\lambda, \epsilon}$ is smooth on $U \times \partial U$. Here, $f \in C^{\infty}(\partial U), g \in C_{0}^{\infty}(U)$ and $G_{\text {free, } \lambda}$ is the kernel of the free resolvent. To establish differentiability by the product rule, it is sufficient to prove the existence of $\frac{\mathrm{d}}{\mathrm{d} \epsilon}\left(\varphi_{\epsilon}^{*} \mathcal{S}_{\lambda, \epsilon} \varphi_{-\epsilon}^{*}\right)$ in the $C^{\infty}$ topology of integral kernels on $U \times \partial U$, and the existence of $\frac{\mathrm{d}}{\mathrm{d} \epsilon}\left(\varphi_{\epsilon}^{*} Q_{\lambda, \epsilon}^{-1} \varphi_{-\epsilon}^{*}\right)$ at $\epsilon=0$ in the weak- $*$-sense. The free resolvent kernel is smooth off the diagonal and therefore the above formula for $\varphi_{\epsilon}^{*} \mathcal{S}_{\lambda, \epsilon} \varphi_{-\epsilon}^{*}$ shows differentiability of the smooth kernel in the parameter $\epsilon$ at $\epsilon=0$ and the classical sense. We are thus left with proving the existence of $\frac{\mathrm{d}}{\mathrm{d} \epsilon}\left(\varphi_{\epsilon}^{*} Q_{\lambda, \epsilon}^{-1} \varphi_{-\epsilon}^{*}\right)$ at $\epsilon=0$ in the weak-*sense. Note that $\varphi_{\epsilon}^{*} Q_{\lambda, \epsilon}^{-1} \varphi_{-\epsilon}^{*}$ is a one-parameter family of maps from $C^{\infty}(\partial U)$ to $C^{\infty}(\partial U)$, i.e. the spaces do not depend on $\epsilon$. Similar to Eq. (14), we have the splitting

$$
\tilde{Q}_{\lambda}=\sum_{j=1}^{N} p_{j} Q_{\lambda} p_{j}, \quad T_{\lambda}=\sum_{j \neq k}^{N} p_{j} Q_{\lambda} p_{k} \quad \text { and } \quad Q_{\lambda}=\tilde{Q}_{\lambda}+T_{\lambda},
$$

where $p_{j}$ are the orthogonal projections $L^{2}(\partial U) \rightarrow L^{2}\left(\partial U_{j}\right)$ and $\partial U_{j}, j=$ $1, \ldots, N$ are the connected components of $\partial U$. Define $\mathcal{Q}_{\lambda, \epsilon}=\varphi_{\epsilon}^{*} Q_{\lambda, \epsilon} \varphi_{-\epsilon}^{*}, \tilde{\mathcal{Q}}_{\lambda, \epsilon}=$ $\varphi_{\epsilon}^{*} \tilde{Q}_{\lambda, \epsilon} \varphi_{-\epsilon}^{*}$ and $\mathcal{T}_{\lambda, \epsilon}=\varphi_{\epsilon}^{*} T_{\lambda, \epsilon} \varphi_{-\epsilon}^{*}$. By the definition of $\tilde{Q}_{\lambda, \epsilon}$, we have

$$
\tilde{\mathcal{Q}}_{\lambda, \epsilon} f\left(x_{i}\right)=\int_{\partial U_{i}} G_{\text {free }, \lambda}\left(\varphi_{\epsilon}\left(x_{i}\right), \varphi_{\epsilon}\left(y_{i}\right)\right) f_{i}\left(y_{i}\right) \varphi_{\epsilon}^{*}\left(\mathrm{~d} \sigma\left(y_{i}\right)\right),
$$

where $f \in C^{\infty}(\partial U)$ and $f_{i}=p_{i} f$. As $\varphi_{\epsilon}$ is a boundary translation flow, we obtain the following relationships.

$$
\tilde{\mathcal{Q}}_{\lambda, \epsilon}=\tilde{Q}_{\lambda, 0}=\tilde{Q}_{\lambda} \quad \text { and } \quad \tilde{\mathcal{Q}}_{\lambda, \epsilon}^{-1}=\tilde{Q}_{\lambda}^{-1} .
$$

Now, from the decomposition of $Q_{\lambda}$ in Eq. (14), one obtains

$$
\left\{\begin{array}{l}
\frac{\mathrm{d}}{\mathrm{d} \epsilon} \mathcal{Q}_{\lambda, \epsilon}=\frac{\mathrm{d}}{\mathrm{d} \epsilon}\left(\tilde{\mathcal{Q}}_{\lambda, \epsilon}+\mathcal{T}_{\lambda, \epsilon}\right)=\frac{\mathrm{d}}{\mathrm{d} \epsilon} \mathcal{T}_{\lambda, \epsilon} \\
\frac{\mathrm{d}}{\mathrm{d} \epsilon} \mathcal{Q}_{\lambda, \epsilon}^{-1}=-\mathcal{Q}_{\lambda, \epsilon}^{-1}\left(\frac{\mathrm{d}}{\mathrm{d} \epsilon} \mathcal{Q}_{\lambda, \epsilon}\right) \mathcal{Q}_{\lambda, \epsilon}^{-1} .
\end{array}\right.
$$

The family $\mathcal{T}_{\lambda, \epsilon}$ is a differentiable family of smoothing operators for sufficiently small $\epsilon$ (i.e. no obstacles are overlapping) and its derivative in $\epsilon$ therefore, by Taylor's remainder estimate, exits as a family of smooth kernels. Hence, $\mathcal{Q}_{\lambda, \epsilon}$ is differentiable in $\epsilon$ at $\epsilon=0$ as a family of operators from $H^{s}(\partial U)$ to $H^{s+1}(\partial U)$ for any $s \in \mathbb{R}$. We now use that $\mathcal{Q}_{\lambda, 0}$ is invertible and the inverse $\mathcal{Q}_{\lambda, 0}^{-1}$ is a pseudodifferential operator of order one, and maps $H^{s}(\partial U)$ to $H^{s-1}(\partial U)$. Since the space of invertible operators is open the inverses $\mathcal{Q}_{\lambda, \epsilon}^{-1}$ exist near $\epsilon=0$ as maps from $H^{s}(\partial U)$ to $H^{s-1}(\partial U)$. Hence, $\mathcal{Q}_{\lambda, \epsilon}^{-1}$ is differentiable in $\epsilon$ at $\epsilon=0$ as a family of operators from $H^{s}(\partial U)$ to $H^{s-1}(\partial U)$. In particular the 
derivatives $\frac{\mathrm{d}}{\mathrm{d} \epsilon} \mathcal{Q}_{\lambda, \epsilon}$ and $\frac{\mathrm{d}}{\mathrm{d} \epsilon} \mathcal{Q}_{\lambda, \epsilon}^{-1}$ exist in the weak-*-sense. Hence, the variational derivative of $R_{\lambda, \epsilon}$ exists in the weak-* sense and it is given by

$$
\delta_{Y} R_{\lambda}=\theta_{Y} R_{\lambda}-Y R_{\lambda}-Y^{\prime} R_{\lambda},
$$

where $Y^{\prime}$ means the action of $Y$ on the second variable. It remains to compute the derivative. To do this, we consider the inhomogeneous problem

$$
E_{\epsilon}\left(u_{\epsilon}, \varphi_{-\epsilon}^{*} v\right)=\int_{U_{\epsilon}} f_{\epsilon}\left(\varphi_{-\epsilon}^{*} v\right) \mathrm{d} x .
$$

Let $\nu$ be the exterior unit normal of $U, y$ be an interior point of $U$ such that $\varphi_{\epsilon}(y)=\tilde{y}$ and $e(u, v)=\nabla u \cdot \nabla v-\lambda^{2} u v$. By applying

$$
\left\{\begin{array}{l}
u_{\epsilon}(x)=G_{\lambda, \epsilon}\left(x, \varphi_{\epsilon}(y)\right), \\
f_{\epsilon}(x)=\delta_{\varphi_{\epsilon}(y)}(x) .
\end{array}\right.
$$

to equation (35), taking derivative in $\epsilon$ of Eq. (35) and using Eq. (30) and Peetre's computations [41], one has

$$
\int_{U} e\left(\delta_{Y} u_{0}, v\right) \mathrm{d} x=\int_{U} e\left(u_{0}, Y(v)\right) \mathrm{d} x-\int_{\partial U} e\left(u_{0}, v\right)\langle Y, \nu\rangle \mathrm{d} \sigma .
$$

Let $v(x)=G_{\lambda, 0}(x, z)$, we have

$$
\int_{U} e(u, v) \mathrm{d} x=u(z)+\int_{\partial U} u(x) \partial_{\nu} G_{\lambda, 0}(x, z) \mathrm{d} \sigma(x) .
$$

Using the symmetric property of $G_{\lambda, \epsilon}(x, y)=G_{\lambda, \epsilon}(y, x)$ and Eqs. (34), (36) and (37), we obtain

$$
\delta_{Y} G_{\lambda, 0}(z, y)+\int_{\partial U} \partial_{\nu}^{\prime} G_{\lambda, 0}(z, x) \delta_{Y} G_{\lambda, 0}(x, y) \mathrm{d} \sigma(x)=0 .
$$

Using the boundary conditions $G_{\lambda, \epsilon}\left(\varphi_{\epsilon}(x), y\right)=0$ for $x \in \partial U$, we arrive at the Hadamard variation formula for the Dirichlet resolvent.

$$
\delta_{Y} G_{\lambda, 0}(z, y)=\int_{\partial U} \partial_{\nu}^{\prime} G_{\lambda, 0}(z, x) \partial_{\nu} G_{\lambda, 0}(x, y)\langle Y, \nu\rangle \mathrm{d} \sigma(x) .
$$

\subsection{Application of the Hadamard Variation Formula to the Relative Resolvent}

We now apply the Hadamard variation formula to our setting with finitely many obstacles, combining the above formulae for $U=\mathcal{E}$ and $U=\mathcal{O}$. We have from Eq. (32)

$$
\delta_{Y} G_{\lambda, 0}(z, y)=\left\{\begin{array}{l}
\int_{\partial X}\left[\left(\partial_{\nu} G_{\lambda, 0}(x, y) \partial_{\nu} G_{\lambda, 0}(x, z)\right)\left\langle Y, \nu_{X}\right\rangle\right]_{+} \mathrm{d} \sigma(x) \quad y, z \in \mathcal{E} \\
\int_{\partial X}\left[\left(\partial_{\nu} G_{\lambda, 0}(x, y) \partial_{\nu} G_{\lambda, 0}(x, z)\right)\left\langle Y, \nu_{X}\right\rangle\right]_{-} \mathrm{d} \sigma(x) \quad y, z \in \mathcal{O}
\end{array}\right.
$$

where $(\cdot)_{+}$means taking limits from $\mathcal{E}$ to the boundary $\partial \mathcal{E}$ and $(\cdot)_{-}$means taking limits from $\mathcal{O}$ to the boundary $\partial \mathcal{O}$. We will now use the variational formula for the relative resolvent to prove the following theorem. Hence, we 
define $\mathcal{O}_{\epsilon}=\varphi_{\epsilon}(\mathcal{O})$ and similarly $\mathcal{O}_{j, \epsilon}=\varphi_{\epsilon}\left(\mathcal{O}_{j}\right)$. In this way, we can define the relative resolvent

$$
R_{\mathrm{rel}, \lambda, \epsilon}=R_{\mathcal{O}_{\epsilon}, \lambda}-R_{\text {free }, \lambda}-\left(\sum_{j=1}^{N} R_{\mathcal{O}_{j, \epsilon}, \lambda}-R_{\text {free }, \lambda}\right)
$$

and its integral kernel $G_{\mathrm{rel}, \lambda, \epsilon}$ depending on the parameter $\epsilon$.

Theorem 5.4. Let $\varphi_{\epsilon}$ be a boundary translation flow, $d \geq 2$ and $\lambda \in \mathfrak{D}_{\nu}$. Then, $R_{\mathrm{rel}, \lambda, \epsilon}$ is for each $\lambda \in \mathfrak{D}_{\nu}$ a $C^{1}$ trace-class operator-valued function of $\epsilon$ near the point $\epsilon=0$. Its derivative $\dot{R}_{\mathrm{rel}, \lambda, 0}$ equals $\delta_{Y} R_{\mathrm{rel}, \lambda}$ and there exists $\delta>0$ such that $L^{2}$-trace-norm of $\dot{R}_{\mathrm{rel}, \lambda, 0}$ is bounded by

$$
\left\|\dot{R}_{\mathrm{rel}, \lambda, 0}\right\|_{1} \leq C_{\nu} \rho(\operatorname{Im} \lambda) e^{-\delta \operatorname{Im} \lambda}, \quad \lambda \in \mathfrak{D}_{\nu} .
$$

Its kernel, $\dot{G}_{\mathrm{rel}, \lambda, 0}=\breve{\dot{R}}_{\mathrm{rel}, \lambda, 0}$, is given by

$$
\begin{aligned}
\dot{G}_{\mathrm{rel}, \lambda, 0}(x, y)= & \sum_{i=1}^{N} \int_{\partial \mathcal{O}_{i}}\left[\left(\partial_{\nu}^{\prime} G_{\mathcal{O}, \lambda, 0}(x, z) \partial_{\nu} G_{\mathcal{O}, \lambda, 0}(z, y)\right.\right. \\
& \left.\left.-\partial_{\nu}^{\prime} G_{\mathcal{O}_{i}, \lambda, 0}(x, z) \partial_{\nu} G_{\mathcal{O}_{i}, \lambda, 0}(z, y)\right)\left\langle Y, \nu_{\mathcal{E}_{i}}\right\rangle\right]_{+} \mathrm{d} \sigma(z)
\end{aligned}
$$

for $x, y \in \mathcal{E}$ or $x, y \in \mathcal{O}$, where $\nu_{\mathcal{E}_{i}}$ is the exterior unit normal of $\mathcal{E}_{i}$ and $\mathcal{E}_{i}=$ $\mathbb{R}^{d} \backslash \overline{\mathcal{O}_{i}}$.

Proof. We start by showing that the family is Fréchet differentiable in the Banach space of trace-class operators with continuous derivative, i.e. the function is $C^{1}$ as a trace-class operator-valued function on $I$, where is a fixed sufficiently small compact interval. As in the previous section we have $R_{\text {rel, } \lambda}=$ $-\mathcal{S}_{\lambda}\left(Q_{\lambda}^{-1}-\tilde{Q}_{\lambda}^{-1}\right) \mathcal{S}_{\lambda}^{t}$. We can decompose its variation form as in the proof of Theorem 5.3

$$
R_{\mathrm{rel}, \lambda, \epsilon}=-\mathcal{S}_{\lambda, \epsilon}\left(Q_{\lambda, \epsilon}^{-1}-\tilde{Q}_{\lambda, \epsilon}^{-1}\right) \mathcal{S}_{\lambda, \epsilon}^{t}=-\mathcal{S}_{\lambda, \epsilon} \varphi_{-\epsilon}^{*} \varphi_{\epsilon}^{*}\left(Q_{\lambda, \epsilon}^{-1}-\tilde{Q}_{\lambda, \epsilon}^{-1}\right) \varphi_{-\epsilon}^{*} \varphi_{\epsilon}^{*} \mathcal{S}_{\lambda, \epsilon}^{t} .
$$

Then, we split the last term into a product of three terms, i.e. $\mathcal{S}_{\lambda, \epsilon} \varphi_{-\epsilon}^{*}, \varphi_{\epsilon}^{*}\left(Q_{\lambda, \epsilon}^{-1}-\right.$ $\left.\tilde{Q}_{\lambda, \epsilon}^{-1}\right) \varphi_{-\epsilon}^{*}$, and $\varphi_{\epsilon}^{*} \mathcal{S}_{\lambda, \epsilon}^{t}$. The first operator $\mathcal{S}_{\lambda, \epsilon} \varphi_{-\epsilon}^{*}$ is given by

$$
\begin{aligned}
\mathcal{S}_{\lambda, \epsilon} \varphi_{-\epsilon}^{*} f(x) & =\int_{\partial \mathcal{O}_{\epsilon}} G_{\text {free }, \lambda}(x, \tilde{y}) f\left(\varphi_{-\epsilon}(\tilde{y})\right) \mathrm{d} \sigma(\tilde{y}) \\
& =\int_{\partial \mathcal{O}} G_{\text {free }, \lambda}\left(x, \varphi_{\epsilon}(y)\right) f(y) \varphi_{\epsilon}^{*}(\mathrm{~d} \sigma(y)) .
\end{aligned}
$$

Since $\partial \mathcal{O}$ is a disjoint union of the components $\partial \mathcal{O}_{j}$ the operators $\mathcal{S}_{\lambda, \epsilon} \varphi_{-\epsilon}^{*}$ splits into a sum $\sum_{j} \mathcal{S}_{j, \lambda, \epsilon}=\sum_{j} T_{j}(\epsilon) \mathcal{S}_{j, \lambda}$, where $\mathcal{S}_{j, \lambda}: L^{2}\left(\partial \mathcal{O}_{j}\right) \rightarrow H^{1}\left(\mathbb{R}^{d}\right)$ and $T_{j}(\epsilon): L^{2}\left(\mathbb{R}^{d}\right) \rightarrow L^{2}\left(\mathbb{R}^{d}\right)$ is the translation $T_{j}(\epsilon) f(x)=f\left(x-Z_{j} \epsilon\right)$. Here, we used the fact that $Z$ is constant and equal to $Z_{j}$ near $\partial \mathcal{O}_{j}$ and that the free Green's function is translation invariant. Since $T_{j}(\epsilon)$ is $C^{1}$ as a family of maps $H^{1}\left(\mathbb{R}^{d}\right) \rightarrow L^{2}\left(\mathbb{R}^{d}\right)$, this shows that $\mathcal{S}_{\lambda, \epsilon} \varphi_{-\epsilon}^{*}$ and its adjoint are $C^{1}$ as families of bounded operators $L^{2}(\partial \mathcal{O}) \rightarrow L^{2}\left(\mathbb{R}^{d}\right)$. As shown in the proof of Theorem 5.3 , the operator $\varphi_{\epsilon}^{*} \tilde{Q}_{\lambda, \epsilon} \varphi_{-\epsilon}^{*}$ is independent of $\epsilon$ and therefore

$$
\varphi_{\epsilon}^{*} Q_{\lambda, \epsilon} \varphi_{-\epsilon}^{*}=\tilde{Q}_{\lambda}+\varphi_{\epsilon}^{*} T_{\lambda, \epsilon} \varphi_{-\epsilon}^{*}=\tilde{Q}_{\lambda}+\mathcal{T}_{\lambda, \epsilon} .
$$


The map $\mathcal{T}_{\lambda, \epsilon}$ has smooth integral kernel that depends smoothly on $\epsilon$ for sufficiently small $\epsilon$. This family is therefore $C^{1}$ as a family of trace-class operators. We temporarily denote $\varphi_{\epsilon}^{*} Q_{\lambda, \epsilon} \varphi_{-\epsilon}^{*}$ by $J_{\epsilon}$, As $G_{\text {free }, \lambda}$ is translation invariant, $\varphi_{\epsilon}^{*} \tilde{Q}_{\lambda, \epsilon} \varphi_{-\epsilon}^{*}$ is independent of $\epsilon$ and hence we denote it by $\tilde{J}$. Then, by the above $J_{\epsilon}=J_{0}+r_{\epsilon}$ and $J_{0}-\tilde{J}$ is trace-class. Moreover, the remainder term $r_{\epsilon}$ is of the form $r_{\epsilon}=\dot{J}_{0} \epsilon+\rho(\epsilon)$, where $\|\rho(\epsilon)\|_{1}=o(\epsilon)$ as $\epsilon \rightarrow 0$. By the Neumann series, we have

$$
J_{\epsilon}^{-1}-\tilde{J}^{-1}=\left(1+J_{0}^{-1} r_{\epsilon}\right)^{-1} J_{0}^{-1}-\tilde{J}^{-1}=J_{0}^{-1}-\tilde{J}^{-1}-J_{0}^{-1} \dot{J}_{0} J_{0}^{-1} \epsilon+\tilde{\rho}(\epsilon),
$$

where again $\|\tilde{\rho}(\epsilon)\|_{1}=o(\epsilon)$. We have used here that trace-class operators form an ideal in the algebra of bounded operators, and the norm estimate $\|A B\|_{1} \leq$ $\|A\|\|B\|_{1}$ holds. We conclude that $\varphi_{\epsilon}^{*}\left(Q_{\lambda, \epsilon}^{-1}-\tilde{Q}_{\lambda, \epsilon}^{-1}\right) \varphi_{-\epsilon}^{*}$ is $C^{1}$ as a family of trace-class operators.

We now compute the derivative in $\epsilon$ of all the three terms. We obtain

$$
\begin{aligned}
\dot{R}_{\mathrm{rel}, \lambda, 0}= & \mathcal{S}_{\lambda} Y\left(Q_{\lambda}^{-1}-\tilde{Q}_{\lambda}^{-1}\right) \mathcal{S}_{\lambda}^{t}-\left.\mathcal{S}_{\lambda} \frac{\mathrm{d}}{\mathrm{d} \epsilon}\left(\varphi_{\epsilon}^{*}\left(Q_{\lambda, \epsilon}^{-1}-\tilde{Q}_{\lambda, \epsilon}^{-1}\right) \varphi_{-\epsilon}^{*}\right)\right|_{\epsilon=0} \\
& \times \mathcal{S}_{\lambda}^{t}-\mathcal{S}_{\lambda}\left(Q_{\lambda}^{-1}-\tilde{Q}_{\lambda}^{-1}\right) Y \mathcal{S}_{\lambda}^{t} .
\end{aligned}
$$

For the derivative of the second term, we have as in Theorem 5.3

$$
\tilde{\mathcal{Q}}_{\lambda, \epsilon} f\left(x_{i}\right)=\int_{\partial \mathcal{O}_{i}} G_{\text {free }, \lambda}\left(\varphi_{\epsilon}\left(x_{i}\right), \varphi_{\epsilon}\left(y_{i}\right)\right) f_{i}\left(y_{i}\right) \varphi_{\epsilon}^{*}\left(\mathrm{~d} \sigma\left(y_{i}\right)\right),
$$

and

$$
\begin{aligned}
& \left.\frac{\mathrm{d}}{\mathrm{d} \epsilon}\left(\varphi_{\epsilon}^{*}\left(Q_{\lambda, \epsilon}^{-1}-\tilde{Q}_{\lambda, \epsilon}^{-1}\right) \varphi_{-\epsilon}^{*}\right)\right|_{\epsilon=0}=\left.\frac{\mathrm{d}}{\mathrm{d} \epsilon} \mathcal{Q}_{\lambda, \epsilon}^{-1}\right|_{\epsilon=0} \\
& =-\left.\left(\mathcal{Q}_{\lambda, \epsilon}^{-1}\left(\frac{\mathrm{d}}{\mathrm{d} \epsilon} \mathcal{T}_{\lambda, \epsilon}\right) \mathcal{Q}_{\lambda, \epsilon}^{-1}\right)\right|_{\epsilon=0} .
\end{aligned}
$$

Therefore, the variation of the relative resolvent is given by

$$
\begin{array}{r}
\dot{R}_{\mathrm{rel}, \lambda, 0}=\mathcal{S}_{\lambda} Y\left(Q_{\lambda}^{-1}-\tilde{Q}_{\lambda}^{-1}\right) \mathcal{S}_{\lambda}^{t}-\left.\mathcal{S}_{\lambda} Q_{\lambda}^{-1} \frac{\mathrm{d}}{\mathrm{d} \epsilon} \mathcal{T}_{\lambda, \epsilon}\right|_{\epsilon=0} Q_{\lambda}^{-1} \mathcal{S}_{\lambda}^{t}-\mathcal{S}_{\lambda}\left(Q_{\lambda}^{-1}-\tilde{Q}_{\lambda}^{-1}\right) Y \mathcal{S}_{\lambda}^{t} \\
=\mathcal{S}_{\lambda} Y\left(Q_{\lambda}^{-1} T_{\lambda} \tilde{Q}_{\lambda}^{-1}\right) \mathcal{S}_{\lambda}^{t}-\mathcal{S}_{\lambda} Q_{\lambda}^{-1} \dot{\mathcal{T}}_{\lambda, 0} Q_{\lambda}^{-1} \mathcal{S}_{\lambda}^{t}-\mathcal{S}_{\lambda}\left(Q_{\lambda}^{-1} T_{\lambda} \tilde{Q}_{\lambda}^{-1}\right) Y \mathcal{S}_{\lambda}^{t}(41)
\end{array}
$$

To estimate the trace-norm of $\dot{R}_{\text {rel }, \lambda, 0}$, note that the first term in the above equation can be estimated by

$\left\|\mathcal{S}_{\lambda} Y\left(Q_{\lambda}^{-1} T_{\lambda} \tilde{Q}_{\lambda}^{-1}\right) \mathcal{S}_{\lambda}^{t}\right\|_{1} \leq\left\|\mathcal{S}_{\lambda} Y Q_{\lambda}^{-1}\right\|_{H^{\frac{1}{2}} \rightarrow L^{2}}\left\|E_{-1}\right\|_{H^{-\frac{1}{2}} \rightarrow H^{\frac{1}{2}}}\left\|E_{1} T_{\lambda}\right\|_{1}\left\|\tilde{Q}_{\lambda}^{-1} \mathcal{S}_{\lambda}^{t}\right\|_{L^{2} \rightarrow H^{-\frac{1}{2}}}$, where $E_{s}=\left(\sqrt{-\Delta_{\partial \mathcal{O}}}+1\right)^{s}$ and $\left\|E_{1} T_{\lambda}\right\|_{1}$ is the trace-norm from $H^{-\frac{1}{2}} \rightarrow H^{-\frac{1}{2}}$. Now by Proposition 4.1, we have

$$
\left\|\mathcal{S}_{\lambda} Y\left(Q_{\lambda}^{-1} T_{\lambda} \tilde{Q}_{\lambda}^{-1}\right) \mathcal{S}_{\lambda}^{t}\right\|_{1} \leq C\left(1+(\operatorname{Im} \lambda)^{2}\right)^{4} \rho(\operatorname{Im} \lambda)\left\|E_{1} T_{\lambda}\right\|_{1} .
$$

The third term in Eq. (41) can be bounded the same as the first term. For the second term, one can estimate it by

$$
\begin{gathered}
\left\|\mathcal{S}_{\lambda} Q_{\lambda}^{-1} \dot{\mathcal{T}}_{\lambda, 0} Q_{\lambda}^{-1} \mathcal{S}_{\lambda}^{t}\right\|_{1} \leq\left\|\mathcal{S}_{\lambda} Q_{\lambda}^{-1}\right\|_{H^{\frac{1}{2}} \rightarrow L^{2}}\left\|E_{-1}\right\|_{H^{-\frac{1}{2}} \rightarrow H^{\frac{1}{2}}}\left\|E_{1} \dot{\mathcal{T}}_{\lambda, 0}\right\|_{1}\left\|Q_{\lambda}^{-1} \mathcal{S}_{\lambda}^{t}\right\|_{L^{2} \rightarrow H^{-\frac{1}{2}}} \\
\leq C\left(1+(\operatorname{Im} \lambda)^{2}\right)^{4} \rho(\operatorname{Im} \lambda)\left\|E_{1} \dot{\mathcal{T}}_{\lambda, 0}\right\|_{1} .
\end{gathered}
$$

Combining Eqs. (42) and (43), one has

$$
\left\|\dot{R}_{\mathrm{rel}, \lambda, 0}\right\|_{1} \leq C\left(1+(\operatorname{Im} \lambda)^{2}\right)^{4} \rho(\operatorname{Im} \lambda)\left(\left\|E_{1} T_{\lambda}\right\|_{1}+\left\|E_{1} \dot{\mathcal{T}}_{\lambda, 0}\right\|_{1}\right) .
$$


In order to prove the bound (39), it suffices to prove that

$$
\left\|\mathcal{T}_{\lambda, \epsilon}\right\|_{1} \leq C e^{-\delta \operatorname{Im} \lambda} \quad \text { and } \quad\left\|\dot{\mathcal{T}}_{\lambda, \epsilon}\right\|_{1} \leq C e^{-\delta \operatorname{Im} \lambda} .
$$

As in Theorem 5.3, $\mathcal{T}_{\lambda, \epsilon}$ is a smoothing operator that depends smoothly on $\epsilon$, as long as we have $\operatorname{dist}\left(\partial \mathcal{O}_{j, \epsilon}, \partial \mathcal{O}_{k, \epsilon}\right)>0$ for all pairs of obstacles. Since the obstacles are compact, $\mathcal{T}_{\lambda, \epsilon}$ is a smoothing operator on compact domains and hence it is also a trace-class operator from $H^{s}(\partial \mathcal{O}) \rightarrow H^{s}(\partial \mathcal{O})$ for all $s \in \mathbb{R}$. This proves the first part of the theorem.

Also, from Theorem 5.3, we know that $\delta_{Y} G_{\mathrm{rel}, \lambda, 0}$ exists in the weak-* sense. By Eqs. (33) and (34), we know that the kernel of $\dot{R}_{\text {rel }, \lambda, 0}$ coincides with $\delta_{Y} G_{\mathrm{rel}, \lambda, 0}$. In other words, the variational derivative exists in a stronger sense. We can therefore apply the variation formula (38) to the relative resolvent, which gives

$$
\begin{aligned}
\dot{R}_{\mathrm{rel}, \lambda, 0}(x, y)= & \delta_{Y} G_{\mathrm{rel}, \lambda, 0}(x, y) \\
= & \delta_{Y}\left(G_{\mathcal{O}, \lambda, 0}-G_{\text {free }}-\sum_{i=1}^{N}\left(G_{\mathcal{O}_{i}, \lambda, 0}-G_{\text {free }}\right)\right)(x, y) \\
= & \delta_{Y} G_{\mathcal{O}, \lambda, 0}(x, y)-\sum_{i=1}^{N} \delta_{Y} G_{\mathcal{O}_{i}, \lambda, 0}(x, y ; z) \\
= & \sum_{i=1}^{N} \int_{\partial \mathcal{O}_{i}}\left[\partial_{\nu}^{\prime} G_{\mathcal{O}, \lambda, 0}(x, q) \partial_{\nu} G_{\mathcal{O}, \lambda, 0}(q, y)\left\langle Y, \nu_{\mathcal{E}_{i}}\right\rangle\right]_{+} \mathrm{d} \sigma(q) \\
& -\sum_{i=1}^{N} \int_{\partial \mathcal{O}_{i}}\left[\partial_{\nu}^{\prime} G_{\mathcal{O}_{i}, \lambda, 0}(x, q) \partial_{\nu} G_{\mathcal{O}_{i}, \lambda, 0}(q, y)\left\langle Y, \nu_{\mathcal{E}_{i}}\right\rangle\right]_{+} \mathrm{d} \sigma(q) \\
= & \sum_{i=1}^{N} \int_{\partial \mathcal{O}_{i}}\left[\left(\partial_{\nu}^{\prime} G_{\mathcal{O}, \lambda, 0}(x, q) \partial_{\nu} G_{\mathcal{O}, \lambda, 0}(q, y)\right.\right. \\
& \left.\left.-\partial_{\nu}^{\prime} G_{\mathcal{O}_{i}, \lambda, 0}(x, q) \partial_{\nu} G_{\mathcal{O}_{i}, \lambda, 0}(q, y)\right)\left\langle Y, \nu_{\mathcal{E}_{i}}\right\rangle\right]_{+} \mathrm{d} \sigma(q) .
\end{aligned}
$$

Since the interior parts of $G_{\mathcal{O}, \lambda, \epsilon}$ and $G_{\mathcal{O}_{j}, \lambda, \epsilon}$ are the same, the interior contributions of $\delta_{Y} G_{\mathcal{O}, \lambda, 0}$ in the expression (38) cancel out with the ones of $\delta_{Y} G_{\mathcal{O}_{j}, \lambda, 0}$. Therefore, we are left with only the exterior contributions as shown in Eq. (40).

Definition 5.5. Let $M$ be a Riemannian manifold with $A$ be an operator on $L^{2}(M)$ with continuous kernel Schwartz kernel $\breve{A} \in C(M \times M)$. For an open subset $V \subset M$, we define the localised trace on $V$ as

$$
\operatorname{Tr}_{V}(A)=\int_{V} \breve{A}(x, x) \mathrm{d} \operatorname{Vol}_{g}(x),
$$

whenever the integral exists. If $A$ is trace-class and has continuous kernel, the trace of $A$ on $L^{2}(M)$ then equals $\operatorname{Tr}_{M}(A)$ by Mercer's theorem. We also write $\operatorname{Tr}_{V}(\breve{A})$.

Our next proposition gives a relationship between the trace of the variation of the relative resolvent with a local trace on the boundary. 
Proposition 5.6. The trace of the variation of the relative resolvent on $L^{2}(X)$ is also given by

$$
\operatorname{Tr}_{X}\left[\delta_{Y} R_{\mathrm{rel}, \lambda}\right]=\frac{1}{2 \lambda} \sum_{i=1}^{N} \operatorname{Tr}_{\partial \mathcal{O}_{i}}\left[\left(\partial_{\nu} \partial_{\nu}^{\prime} \frac{\partial G_{\mathcal{O}, \lambda}}{\partial \lambda}-\partial_{\nu} \partial_{\nu}^{\prime} \frac{\partial G_{\mathcal{O}_{i}, \lambda}}{\partial \lambda}\right)\langle Y, \nu\rangle\right]_{+}
$$

Proof. Let $\gamma_{\mathcal{E}, \nu}: H^{\frac{3}{2}}(\mathcal{E}) \rightarrow L^{2}(\partial \mathcal{O})$ be the Sobolev trace after taking the exterior normal derivative ( $\nu$ is the exterior normal vector field) and $\mathcal{B}_{\mathcal{O}, \lambda}$ : $L^{2}(\partial \mathcal{O}) \rightarrow L^{2}\left(\mathbb{R}^{d}\right)$ is defined as $\mathcal{B}_{\mathcal{O}, \lambda}=R_{\mathcal{O}, \lambda} \circ \gamma_{\mathcal{E}, \nu}^{*}$. To see that this map is well defined, we note that

$$
R_{\mathcal{O}, \lambda} \gamma_{\mathcal{E}, \nu}^{*}=R_{\text {free }, \lambda} \gamma_{\mathcal{E}, \nu}^{*}-\mathcal{S}_{\mathcal{O}, \lambda} Q_{\mathcal{O}, \lambda}^{-1} \mathcal{S}_{\mathcal{O}, \lambda}^{t} \gamma_{\mathcal{E}, \nu}^{*}
$$

where $\mathcal{S}_{\mathcal{O}, \lambda}$ and $Q_{\mathcal{O}, \lambda}$ are the same as $\mathcal{S}_{\lambda}$ and $Q_{\lambda}$ defined in (22), but with emphasis on the dependence on $\mathcal{O}$. Then, $R_{\text {free }, \lambda} \gamma_{\mathcal{E}, \nu}^{*}$ maps $L^{2}(\partial \Omega)$ continuously to $L^{2}\left(\mathbb{R}^{d}\right)$. The operator $\mathcal{S}_{\lambda}^{t} \gamma_{\mathcal{E}, \nu}^{*}$ is the double layer operator on the boundary $\partial \mathcal{O}$ and maps continuously $L^{2}(\partial \Omega) \rightarrow L^{2}(\partial \Omega)$ (see for example [52]). Since $Q_{\lambda}^{-1}$ is a pseudodifferential operator of order one, and $\mathcal{S}_{\lambda}$ continuously maps $H^{-1}(\partial \mathcal{O})$ to $H^{\frac{1}{2}}\left(\mathbb{R}^{d}\right) \subset L^{2}\left(\mathbb{R}^{d}\right)$ we see that $\mathcal{B}_{\mathcal{O}, \lambda}: L^{2}(\partial \mathcal{O}) \rightarrow L^{2}\left(\mathbb{R}^{d}\right)$ is indeed well defined and continuous.

Similarly, we define $\gamma_{\mathcal{O}_{i}, \nu}$ and $\mathcal{B}_{\mathcal{O}_{i}, \lambda}$. Let $p_{i}$ be the orthogonal projection $L^{2}(\partial \mathcal{O}) \rightarrow L^{2}\left(\partial \mathcal{O}_{i}\right)$. Then, from $(44)$ then have for $\left(\mathcal{B}_{\mathcal{O}, \lambda}-\mathcal{B}_{\mathcal{O}_{i}, \lambda}\right) p_{i}$ the representation

$$
\begin{aligned}
\left(\mathcal{B}_{\mathcal{O}, \lambda}-\mathcal{B}_{\mathcal{O}_{i}, \lambda}\right) p_{i} & =S_{\lambda}\left(Q_{\lambda}^{-1}-Q_{\mathcal{O}_{i}, \lambda}^{-1} p_{i}\right) S_{\lambda}^{t} \gamma_{\mathcal{E}, \nu}^{*} p_{i} \\
& =S_{\lambda}\left(Q_{\lambda}^{-1}-\tilde{Q}_{\lambda}^{-1} p_{i}\right) S_{\lambda}^{t} \gamma_{\mathcal{E}, \nu}^{*} p_{i} \\
& =S_{\lambda}\left(Q_{\lambda}^{-1}-\tilde{Q}_{\lambda}^{-1}\right) S_{\lambda}^{t} \gamma_{\mathcal{E}, \nu}^{*} p_{i}+\sum_{k \neq i} S_{\lambda} \tilde{Q}_{\lambda}^{-1} p_{k} S_{\lambda}^{t} \gamma_{\mathcal{E}, \nu}^{*} p_{i}
\end{aligned}
$$

where $Q_{\mathcal{O}_{i}, \lambda}$ is the operator $Q_{\lambda}$ in Eq. (22) when $\mathcal{O}$ is replaced by $\mathcal{O}_{i}$ in the definition. As in (14) we have used the decomposition $Q_{\lambda}=\tilde{Q}_{\lambda}+T_{\lambda}$. Since $T_{\lambda}$ is smoothing, so is $Q_{\lambda}^{-1}-\tilde{Q}_{\lambda}^{-1}=-Q_{\lambda}^{-1} T_{\lambda} \tilde{Q}_{\lambda}^{-1}$. Similarly, as the free Green's function is smooth off the diagonal the operator $p_{k} S_{\lambda}^{t} \gamma_{\mathcal{E}, \nu}^{*} p_{i}$ has smooth integral kernel for $k \neq i$. In particular, these operators are trace-class as maps from $L^{2}(\partial \mathcal{O})$ to $L^{2}(\partial \mathcal{O})$. Since $S_{\lambda}$ as well as $S_{\lambda} \tilde{Q}_{\lambda}^{-1}$ is bounded from $L^{2}(\partial \mathcal{O})$ to $L^{2}\left(\mathbb{R}^{d}\right)$ this shows that for every $i \in\{1, \ldots, N\}$ the operator $\left(\mathcal{B}_{\mathcal{O}, \lambda}-\mathcal{B}_{\mathcal{O}_{i}, \lambda}\right) p_{i}$ is nuclear.

Equation (40) can be rewritten as

$$
\delta_{Y} R_{\mathrm{rel}, \lambda}=\sum_{i=1}^{N}\left(\mathcal{B}_{\mathcal{O}, \lambda} Y_{i} \mathcal{B}_{\mathcal{O}, \lambda}^{*}-\mathcal{B}_{\mathcal{O}_{i}, \lambda} Y_{i} \mathcal{B}_{\mathcal{O}_{i}, \lambda}^{*}\right)
$$


where $Y_{i}=\left.\left\langle Y, \nu_{\mathcal{E}_{i}}\right\rangle\right|_{\partial \mathcal{O}_{i}}$ is viewed as a multiplication operator acting on $L^{2}\left(\partial \mathcal{O}_{i}\right)$ $\subset L^{2}(\partial \mathcal{O})$. Taking the trace, we have

$$
\begin{aligned}
\operatorname{Tr}\left(\delta_{Y} R_{\mathrm{rel}, \lambda}\right) & =\sum_{i=1}^{N} \operatorname{Tr}\left(\mathcal{B}_{\mathcal{O}, \lambda} Y_{i} \mathcal{B}_{\mathcal{O}, \lambda}^{*}-\mathcal{B}_{\mathcal{O}_{i}, \lambda} Y_{i} \mathcal{B}_{\mathcal{O}_{i}, \lambda}^{*}\right) \\
& =\sum_{i=1}^{N} \operatorname{Tr}\left(\left(\mathcal{B}_{\mathcal{O}, \lambda}-\mathcal{B}_{\mathcal{O}_{i}, \lambda}\right) Y_{i} \mathcal{B}_{\mathcal{O}, \lambda}^{*}+\mathcal{B}_{\mathcal{O}_{i}, \lambda} Y_{i}\left(\mathcal{B}_{\mathcal{O}, \lambda}^{*}-\mathcal{B}_{\mathcal{O}_{i}, \lambda}^{*}\right)\right) \\
& =\sum_{i=1}^{N} \operatorname{Tr}\left(\mathcal{B}_{\mathcal{O}, \lambda}^{*}\left(\mathcal{B}_{\mathcal{O}, \lambda}-\mathcal{B}_{\mathcal{O}_{i}, \lambda}\right) Y_{i}+Y_{i}\left(\mathcal{B}_{\mathcal{O}_{, \lambda}}^{*}-\mathcal{B}_{\mathcal{O}_{i}, \lambda}^{*}\right) \mathcal{B}_{\mathcal{O}_{i}, \lambda}\right) \\
& =\sum_{i=1}^{N} \operatorname{Tr}\left(\left(\mathcal{B}_{\mathcal{O}_{, \lambda}}^{*} \mathcal{B}_{\mathcal{O}, \lambda}-\mathcal{B}_{\mathcal{O}_{i}, \lambda}^{*} \mathcal{B}_{\mathcal{O}_{i}, \lambda}\right) Y_{i}\right) .
\end{aligned}
$$

Here, the cyclic permutation under the trace is justified because of the nuclearity of $\left(\mathcal{B}_{\mathcal{O}, \lambda}-\mathcal{B}_{\mathcal{O}_{i}, \lambda}\right) p_{i}$. Since $\mathcal{B}_{\mathcal{O}, \lambda}^{*} \mathcal{B}_{\mathcal{O}, \lambda}=\frac{1}{2 \lambda} \gamma_{\mathcal{E}, \nu} \frac{\mathrm{d}}{\mathrm{d} \lambda} R_{\mathcal{O}, \lambda} \gamma_{\mathcal{E}, \nu}^{*}$ its integral kernel is $\frac{1}{2 \lambda} \partial_{\nu} \partial_{\nu}^{\prime} \frac{\partial G_{\mathcal{O}, \lambda}}{\partial \lambda}$. We then obtain

$$
\begin{aligned}
\operatorname{Tr}\left(\delta_{Y} R_{\mathrm{rel}, \lambda}\right) & =\sum_{i=1}^{N} \operatorname{Tr}\left(\left(\mathcal{B}_{\mathcal{O}, \lambda}^{*} \mathcal{B}_{\mathcal{O}, \lambda}-\mathcal{B}_{\mathcal{O}_{i}, \lambda}^{*} \mathcal{B}_{\mathcal{O}_{i}, \lambda}\right) Y_{i}\right) \\
& =\frac{1}{2 \lambda} \sum_{i=1}^{N} \operatorname{Tr}_{\partial \mathcal{O}_{i}}\left[\left(\partial_{\nu} \partial_{\nu}^{\prime} \frac{\partial G_{\mathcal{O}, \lambda}}{\partial \lambda}-\partial_{\nu} \partial_{\nu}^{\prime} \frac{\partial G_{\mathcal{O}_{i}, \lambda}}{\partial \lambda}\right) Y_{i}\right]_{+} \\
& =\frac{1}{2 \lambda} \sum_{i=1}^{N} \operatorname{Tr}_{\partial \mathcal{O}_{i}}\left[\left(\partial_{\nu} \partial_{\nu}^{\prime} \frac{\partial G_{\mathcal{O}, \lambda}}{\partial \lambda}-\partial_{\nu} \partial_{\nu}^{\prime} \frac{\partial G_{\mathcal{O}_{i}, \lambda}}{\partial \lambda}\right)\langle Y, \nu\rangle\right]_{+}
\end{aligned}
$$

By Theorem 3.2, one can define trace-class operators $g_{\text {rel }}=D_{f}=\frac{\mathrm{i}}{\pi} \int_{\tilde{\Gamma}} \lambda f(\lambda) R_{\mathrm{rel}, \lambda} \mathrm{d} \lambda$ for $f(\lambda)=g\left(\lambda^{2}\right)$ and $g \in \mathcal{P}_{\theta}$. We now have the following.

Proposition 5.7. Let $g \in \mathcal{P}_{\theta}$ and $\varphi_{\epsilon}$ be a boundary translation flow. Then, $\delta_{Y} g_{\mathrm{rel}}$ is a $C^{1}$ trace-class operator-valued function of $\epsilon$ near the point $\epsilon=0$. Its derivative $\dot{g}_{\text {rel }}=\delta_{Y} g_{\text {rel }}$ satisfies

$$
\operatorname{Tr}\left(\delta_{Y} g_{\mathrm{rel}}\right)=-\sum_{i=1}^{N} \operatorname{Tr}_{\partial \mathcal{O}_{i}}\left[\partial_{\nu} \partial_{\nu}^{\prime}\left(\breve{g}_{\mathcal{O}}^{\prime}-\breve{g}_{\mathcal{O}_{i}}^{\prime}\right)\langle Y, \nu\rangle\right]_{+},
$$

where $g^{\prime}(z)=\frac{\mathrm{d} g}{\mathrm{~d} z}(z)$.

Proof. By Theorem 5.4, the operator $R_{\text {rel }}(z)$ is, for fixed $z \notin \mathfrak{S}_{\theta}$, in the Banach space $C^{1}\left(I, \mathcal{L}_{1}\right)$ of trace-class operator-valued $C^{1}$-functions on a compact interval $I$ containing zero. Differentiation defines a closed operator from on $C\left(I, \mathcal{L}_{1}\right)$ with domain $C^{1}\left(I, \mathcal{L}_{1}\right)$. By Theorem 5.4 , the derivative of $R_{\mathrm{rel}, \lambda}$ is integrable in $\lambda$. An application of Hille's theorem to the Bochner integral defining $g_{\text {rel }}$ in the Banach space of trace-class operators shows that differentiation in $\epsilon$ commutes with integration. We therefore know that $g_{\mathrm{rel}}$ is differentiable and

$$
\delta_{Y} g_{\mathrm{rel}}=\frac{\mathrm{i}}{2 \pi} \int_{\Gamma} g(z) \delta_{Y} R_{\mathrm{rel}}(z) \mathrm{d} z=\frac{\mathrm{i}}{\pi} \int_{\tilde{\Gamma}} \lambda f(\lambda) \delta_{Y} R_{\mathrm{rel}, \lambda} \mathrm{d} \lambda \quad \text { with } \quad R_{\mathrm{rel}}\left(\lambda^{2}\right)=R_{\mathrm{rel}, \lambda} .
$$


Let $f(\lambda)=g\left(\lambda^{2}\right)$. Using Proposition 5.6 and integration by parts in $\lambda$, we have

$$
\begin{aligned}
\operatorname{Tr}\left(\delta_{Y} g_{\mathrm{rel}}\right) & =\frac{\mathrm{i}}{\pi} \int_{\tilde{\Gamma}} \lambda f(\lambda) \operatorname{Tr}\left(\delta_{Y} R_{\mathrm{rel}, \lambda}\right) \mathrm{d} \lambda \\
& =\frac{\mathrm{i}}{2 \pi} \int_{\tilde{\Gamma}} f(\lambda) \sum_{i=1}^{N} \operatorname{Tr}_{\partial \mathcal{O}_{i}}\left[\left(\partial_{\nu} \partial_{\nu}^{\prime} \frac{\partial G_{\mathcal{O}, \lambda}}{\partial \lambda}-\partial_{\nu} \partial_{\nu}^{\prime} \frac{\partial G_{\mathcal{O}_{i}, \lambda}}{\partial \lambda}\right)\langle Y, \nu\rangle\right]_{+} \mathrm{d} \lambda \\
& =-\sum_{i=1}^{N} \operatorname{Tr}_{\partial \mathcal{O}_{i}}\left[\partial_{\nu} \partial_{\nu}^{\prime}\left(\breve{g}_{\mathcal{O}}^{\prime}-\breve{g}_{\mathcal{O}_{i}}^{\prime}\right)\langle Y, \nu\rangle\right]_{+} .
\end{aligned}
$$

In the special case $g(z)=\sqrt{z+m^{2}}$, Proposition 5.7 shows differentiability of $H_{\text {rel }}$ with respect to $\epsilon$ in the space of trace-class operators at $\epsilon=0$. Using Theorem 3.7 and differentiating under the trace then gives the following theorem.

Theorem 5.8. The variation of the relative energy is given by $\delta_{Y} E_{\mathrm{rel}}=\frac{1}{2} \operatorname{Tr}\left(\delta_{Y}\right.$ $\left.H_{\text {rel }}\right)$.

We will now use the Hadamard variation formula to compute this variation.

\subsection{Variation of the Klein-Gordon Energy}

Theorem 5.9. Let $Y$ be a smooth boundary translation vector field. The variation of the Klein-Gordon energy generated by $Y$ is equal to the boundary integral of its spatial tensor contracted with $Y$. That is

$$
\delta_{Y} E_{\mathrm{rel}}=-\int_{\partial \mathcal{O}}\left(T_{\mathrm{rel}}\right)_{i j} \nu^{i} Y^{j} \mathrm{~d} \sigma,
$$

where the integration on the right-hand side is at the exterior boundary and $\nu$ is the exterior normal for $\mathcal{E}$.

Proof. From Eq. (5), we have

$$
\begin{aligned}
\int_{\partial \mathcal{O}}\left(T_{\text {rel }}\right)_{i j} \nu^{i} Y^{j} \mathrm{~d} \sigma & =\sum_{p=1}^{N} \int_{\partial \mathcal{O}_{p}}\left(T_{\text {rel }}\right)_{i j} \nu^{i} Y^{j} \mathrm{~d} \sigma \\
& =\sum_{p=1}^{N} \int_{\partial \mathcal{O}_{p}}\left(\left(T_{\text {ren }}\right)_{\mathcal{O}}-\sum_{q=1}^{N}\left(T_{\text {ren }}\right)_{\mathcal{O}_{q}}\right)_{i j} \nu^{i} Y^{j} \mathrm{~d} \sigma .
\end{aligned}
$$

We know that

- $\left(\left(T_{\text {ren }}\right)_{\mathcal{O}_{p}}\right)_{i j}$ is smooth on a neighbourhood of $\mathcal{O}_{q}$ for $p \neq q$ (by Theorem $2.6)$

- $\left(\left(T_{\text {ren }}\right)_{\mathcal{O}_{p}}\right)_{i j}$ is divergence-free (by Theorem 2.6),

- $Y$ is constant on a neighbourhood of $\mathcal{O}_{q}$ (by assumptions) for all $q \epsilon$ $\{1, \ldots N\}$.

Therefore, $F_{i}=\left(\left(T_{\mathrm{ren}}\right)_{\mathcal{O}_{p}}\right)_{i j} Y^{j}$ is smooth and divergence-free on a neighbourhood of $\mathcal{O}_{q}$. In other words, we have

$$
\int_{\partial \mathcal{O}_{q}}\left(\left(T_{\mathrm{ren}}\right)_{\mathcal{O}_{p}}\right)_{i j} \nu^{i} Y^{j} \mathrm{~d} \sigma=0 \quad \text { for } \quad q \neq p .
$$


That is

$$
\int_{\partial \mathcal{O}}\left(T_{\text {rel }}\right)_{i j} \nu^{i} Y^{j} \mathrm{~d} \sigma=\sum_{p=1}^{N} \int_{\partial \mathcal{O}_{p}}\left(\left(T_{\text {ren }}\right)_{\mathcal{O}}-\left(T_{\text {ren }}\right)_{\mathcal{O}_{p}}\right)_{i j} \nu^{i} Y^{j} \mathrm{~d} \sigma .
$$

As $\left(\left(T_{\text {ren }}\right)_{\mathcal{O}}-\left(T_{\text {ren }}\right)_{\mathcal{O}_{p}}\right)_{i j}$ is vanishing at the boundary $\partial \mathcal{O}_{p}$, we have

$$
\left(\left(T_{\text {ren }}\right)_{\mathcal{O}}-\left(T_{\text {ren }}\right)_{\mathcal{O}_{p}}\right)_{i j} Y^{j}=\left(\left(T_{\text {ren }}\right)_{\mathcal{O}}-\left(T_{\text {ren }}\right)_{\mathcal{O}_{p}}\right)_{i j} \nu^{j}\langle Y, \nu\rangle \quad \text { on } \quad \partial \mathcal{O}_{p}
$$

Now, from Theorem 2.4 we get

$$
\begin{aligned}
\sum_{p=1}^{N} & \int_{\partial \mathcal{O}_{p}}\left(\left(T_{\text {ren }}\right)_{\mathcal{O}}-\left(T_{\text {ren }}\right)_{\mathcal{O}_{p}}\right)_{i j} \nu^{i} Y^{j} \mathrm{~d} \sigma \\
= & \sum_{p=1}^{N} \int_{\partial \mathcal{O}_{p}}\left(\left(T_{\text {ren }}\right)_{\mathcal{O}}-\left(T_{\text {ren }}\right)_{\mathcal{O}_{p}}\right)_{i j} \nu^{i} \nu^{j}\langle Y, \nu\rangle \mathrm{d} \sigma \\
= & \sum_{p=1}^{N} \int_{\partial \mathcal{O}_{p}} \frac{1}{2}\left\{\left[\left.\left[\partial_{\nu} \partial_{\nu}^{\prime}\left(\breve{H}_{\mathcal{O}}^{-1}-\breve{H}_{\text {free }}^{-1}\right)\right]\right|_{\Delta}-\frac{1}{4} \Delta\left[\left.\left(\breve{H}_{\mathcal{O}}^{-1}-\breve{H}_{\text {free }}^{-1}\right)\right|_{\Delta}\right]\right]\right. \\
& \left.-\left[\left.\left[\partial_{\nu} \partial_{\nu}^{\prime}\left(\breve{H}_{\mathcal{O}_{p}}^{-1}-\breve{H}_{\text {free }}^{-1}\right)\right]\right|_{\Delta}-\frac{1}{4} \Delta\left[\left.\left(\breve{H}_{\mathcal{O}_{p}}^{-1}-\breve{H}_{\text {free }}^{-1}\right)\right|_{\Delta}\right]\right]\right\}\langle Y, \nu\rangle \mathrm{d} \sigma \\
= & \frac{1}{2} \sum_{p=1}^{N} \int_{\partial \mathcal{O}_{p}}\left[\left.\left[\partial_{\nu} \partial_{\nu}^{\prime}\left(\breve{H}_{\mathcal{O}}^{-1}-\breve{H}_{\mathcal{O}_{p}}^{-1}\right)\right]\right|_{\Delta}-\frac{1}{4} \Delta\left[\left.\left(\breve{H}_{\mathcal{O}}^{-1}-\breve{H}_{\mathcal{O}_{p}}^{-1}\right)\right|_{\Delta}\right]\right]\langle Y, \nu\rangle \mathrm{d} \sigma .
\end{aligned}
$$

Altogether, we have

$$
\begin{aligned}
\int_{\partial \mathcal{O}}\left(T_{\mathrm{rel}}\right)_{i j} \nu^{i} Y^{j} \mathrm{~d} \sigma= & \frac{1}{2} \sum_{p=1}^{N} \int_{\partial \mathcal{O}_{p}}\left[\left.\left[\partial_{\nu} \partial_{\nu}^{\prime}\left(\breve{H}_{\mathcal{O}}^{-1}-\breve{H}_{\mathcal{O}_{p}}^{-1}\right)\right]\right|_{\Delta}\right. \\
& \left.-\frac{1}{4} \Delta\left[\left.\left(\breve{H}_{\mathcal{O}}^{-1}-\breve{H}_{\mathcal{O}_{p}}^{-1}\right)\right|_{\Delta}\right]\right]\langle Y, \nu\rangle \mathrm{d} \sigma .
\end{aligned}
$$

The second term in the above equation can be expressed as

$$
\begin{aligned}
\operatorname{Tr}_{\partial \mathcal{O}_{p}}\left[\Delta\left(\left.\left(\breve{H}_{\mathcal{O}}^{-1}-\breve{H}_{\mathcal{O}_{p}}^{-1}\right)\right|_{\Delta}\right)\right] & =-\frac{\mathrm{i}}{2 \pi} \int_{\Gamma} \frac{1}{\sqrt{z}} \operatorname{Tr}_{\partial \mathcal{O}_{p}}\left[\partial_{k} \partial_{k}\left(\left.\left(G_{\mathcal{O}}(z)-G_{\mathcal{O}_{p}}(z)\right)\right|_{\Delta}\right)\right] \mathrm{d} z \\
& =-\frac{\mathrm{i}}{2 \pi} \int_{\Gamma} \frac{1}{\sqrt{z}} \operatorname{Tr}_{\partial \mathcal{O}_{p}}\left[\left.2 \partial_{k} \partial_{k}^{\prime}\left(G_{\mathcal{O}}(z)-G_{\mathcal{O}_{p}}(z)\right)\right|_{\Delta}\right] \mathrm{d} z,
\end{aligned}
$$

where we used the properties (11) and (30) of $G_{\mathcal{O}}\left(\lambda^{2}\right)=G_{\mathcal{O}, \lambda}$ and $G_{\mathcal{O}_{p}}\left(\lambda^{2}\right)=$ $G_{\mathcal{O}_{p}, \lambda}$. Now we obtain

$$
\operatorname{Tr}_{\partial \mathcal{O}_{p}}\left[\Delta\left(\left.\left(\breve{H}_{\mathcal{O}}^{-1}-\breve{H}_{\mathcal{O}_{p}}^{-1}\right)\right|_{\Delta}\right)\right]=2 \operatorname{Tr}_{\partial \mathcal{O}_{p}}\left[\left.\left[\partial_{\nu} \partial_{\nu}^{\prime}\left(\breve{H}_{\mathcal{O}}^{-1}-\breve{H}_{\mathcal{O}_{p}}^{-1}\right)\right]\right|_{\Delta}\right] .
$$

Equations (45) and (46) imply

$$
\int_{\partial \mathcal{O}}\left(T_{\mathrm{rel}}\right)_{i j} \nu^{i} Y^{j} \mathrm{~d} \sigma=\frac{1}{4} \sum_{p=1}^{N} \int_{\partial \mathcal{O}_{p}}\left[\left.\left[\partial_{\nu} \partial_{\nu}^{\prime}\left(\breve{H}_{\mathcal{O}}^{-1}-\breve{H}_{\mathcal{O}_{p}}^{-1}\right)\right]\right|_{\Delta}\right]\langle Y, \nu\rangle \mathrm{d} \sigma .
$$

Since $\int_{\partial \mathcal{O}}\left(T_{\mathrm{rel}}\right)_{i j} \nu^{i} Y^{j} \mathrm{~d} \sigma$ is integrating at the exterior boundary, we have

$$
\int_{\partial \mathcal{O}}\left(T_{\mathrm{rel}}\right)_{i j} \nu^{i} Y^{j} \mathrm{~d} \sigma=\frac{1}{4} \sum_{p=1}^{N} \int_{\partial \mathcal{O}_{p}}\left[\left.\left[\partial_{\nu} \partial_{\nu}^{\prime}\left(\breve{H}_{\mathcal{O}}^{-1}-\breve{H}_{\mathcal{O}_{p}}^{-1}\right)\right]\right|_{\Delta}\right]_{+}\langle Y, \nu\rangle \mathrm{d} \sigma .
$$


Applying Proposition 5.7 to $g(z)=\sqrt{z+m^{2}}$, we have

$$
\begin{aligned}
\operatorname{Tr}\left(\delta_{Y} H_{\mathrm{rel}}\right)= & \operatorname{Tr}\left(\delta_{Y}\left(H_{\mathcal{O}}-\sum_{p=1}^{N} H_{\mathcal{O}_{p}}\right)\right) \\
= & \left(\operatorname{Tr}_{\mathcal{E}}+\operatorname{Tr}_{\mathcal{O}}\right)\left[\delta_{Y}\left(H_{\mathcal{O}}-\sum_{p=1}^{N} H_{\mathcal{O}_{p}}\right)\right] \\
= & -\frac{1}{2}\left\{\operatorname{Tr}_{\partial \mathcal{O}}\left[\partial_{\nu} \partial_{\nu}^{\prime} \breve{H}_{\mathcal{O}}^{-1}\langle Y, \nu\rangle\right]_{+}-\sum_{p=1}^{N} \operatorname{Tr}_{\partial \mathcal{O}_{p}}\left[\partial_{\nu} \partial_{\nu}^{\prime} \breve{H}_{\mathcal{O}_{p}}^{-1}\langle Y, \nu\rangle\right]_{+}\right\} \\
& -\frac{1}{2}\left\{\operatorname{Tr}_{\partial \mathcal{O}}\left[\partial_{\nu} \partial_{\nu}^{\prime} \breve{H}_{\mathcal{O}}^{-1}\langle Y, \nu\rangle\right]_{-}-\sum_{p=1}^{N} \operatorname{Tr}_{\partial \mathcal{O}_{p}}\left[\partial_{\nu} \partial_{\nu}^{\prime} \breve{H}_{\mathcal{O}_{p}}^{-1}\langle Y, \nu\rangle\right]_{-}\right\} .
\end{aligned}
$$

Now $\operatorname{Tr}_{\partial \mathcal{O}}\left[\partial_{\nu} \partial_{\nu}^{\prime} \breve{H}_{\mathcal{O}}^{-1}\langle Y, \nu\rangle\right]_{-}-\sum_{p=1}^{N} \operatorname{Tr}_{\partial \mathcal{O}_{p}}\left[\partial_{\nu} \partial_{\nu}^{\prime} \breve{H}_{\mathcal{O}_{p}}^{-1}\langle Y, \nu\rangle\right]_{-}=0$ as $Y$ is a boundary translation vector field. Therefore, we have

$$
\begin{aligned}
\operatorname{Tr}\left(\delta_{Y} H_{\mathrm{rel}}\right) & =-\frac{1}{2}\left\{\operatorname{Tr}_{\partial \mathcal{O}}\left[\partial_{\nu} \partial_{\nu}^{\prime} \breve{H}_{\mathcal{O}}^{-1}\langle Y, \nu\rangle\right]_{+}-\sum_{p=1}^{N} \operatorname{Tr}_{\partial \mathcal{O}_{p}}\left[\partial_{\nu} \partial_{\nu}^{\prime} \breve{H}_{\mathcal{O}_{p}}^{-1}\langle Y, \nu\rangle\right]_{+}\right\} \\
& =-\frac{1}{2} \sum_{p=1}^{N}\left\{\operatorname{Tr}_{\partial \mathcal{O}_{p}}\left[\partial_{\nu} \partial_{\nu}^{\prime} \breve{H}_{\mathcal{O}}^{-1}\langle Y, \nu\rangle\right]_{+}-\operatorname{Tr}_{\partial \mathcal{O}_{p}}\left[\partial_{\nu} \partial_{\nu}^{\prime} \breve{H}_{\mathcal{O}_{p}}^{-1}\langle Y, \nu\rangle\right]_{+}\right\} \\
& =-\frac{1}{2} \sum_{p=1}^{N}\left\{\operatorname{Tr}_{\partial \mathcal{O}_{p}}\left[\partial_{\nu} \partial_{\nu}^{\prime}\left(\breve{H}_{\mathcal{O}}^{-1}-\breve{H}_{\mathcal{O}_{p}}^{-1}\right)\langle Y, \nu\rangle\right]_{+}\right\} \\
& =-\frac{1}{2} \sum_{p=1}^{N} \int_{\partial \mathcal{O}_{p}}\left[\left[\partial_{\nu} \partial_{\nu}^{\prime}\left(\breve{H}_{\mathcal{O}}^{-1}-\breve{H}_{\mathcal{O}_{p}}^{-1}\right)\right]_{\Delta}\right]_{+}\langle Y, \nu\rangle \mathrm{d} \sigma .
\end{aligned}
$$

That is

$$
\operatorname{Tr}\left(\delta_{Y} H_{\text {rel }}\right)=-\frac{1}{2} \sum_{p=1}^{N} \int_{\partial \mathcal{O}_{p}}\left[\left[\partial_{\nu} \partial_{\nu}^{\prime}\left(\breve{H}_{\mathcal{O}}^{-1}-\breve{H}_{\mathcal{O}_{p}}^{-1}\right)\right]_{\Delta}\right]_{+}\langle Y, \nu\rangle \mathrm{d} \sigma .
$$

Finally, Eqs. (47), (48) and Theorem 5.8 complete the proof.

An application of an analogue of Theorem 5.9 to calculate the Casimir force in dimension one can be found in Appendix 7. Finally, we have the following theorem.

Theorem 5.10. Let $T_{\text {ren }}$ be the renormalised stress-energy tensor in Theorem 2.3 and let $Y$ be a boundary translation flow as in Theorem 5.9. We assume further that $Y$ is constant near $\mathcal{O}_{p}$ for some $p \in\{1, \ldots, N\}$ and vanishes near $\mathcal{O}_{q}$ if $q \neq p$. Let $S$ be any smooth hypersurface in $\mathcal{E}$ that is homologous to $\partial \mathcal{O}_{p}$ in $\overline{\mathcal{E}}$ and let $\nu$ be the exterior normal vector field of $S$. Then, the variation of the relative energy generated by $Y$ is equal to

$$
\delta_{Y} E_{\text {rel }}=\int_{S}\left(T_{\text {ren }}\right)_{i j} \nu^{i} Z^{j} \mathrm{~d} \sigma,
$$

where $Z$ is the unique constant vector field on $\mathbb{R}^{d}$ whose restriction to $\partial \mathcal{O}_{q}$ equals $Y$. 
Proof. As in the proof of Theorem 5.9, we have

$$
\begin{aligned}
\int_{\partial \mathcal{O}}\left(T_{\text {rel }}\right)_{i j} \nu^{i} Y^{j} \mathrm{~d} \sigma & =\int_{\partial \mathcal{O}_{p}}\left(\left(T_{\text {ren }}\right)_{\mathcal{O}}-\left(T_{\text {ren }}\right)_{\mathcal{O}_{p}}\right)_{i j} \nu^{i} Z^{j} \mathrm{~d} \sigma \\
& =\int_{\partial \mathcal{O}_{p}}\left(\left(T_{\text {ren }}\right)_{\mathcal{O}}-\left(T_{\text {ren }}\right)_{\mathcal{O}_{p}}\right)_{i j} \nu^{i} \nu^{j}\langle Z, \nu\rangle \mathrm{d} \sigma .
\end{aligned}
$$

We also know that $\partial \mathcal{O}_{p}$ is homologous to $S_{R}$, a sphere with sufficiently large radius $R$ in $\mathbb{R}^{d} \backslash \mathcal{O}_{p}$. Because $\left(T_{\text {ren }}\right)_{\mathcal{O}_{p}}$ is divergent-free, one has

$$
\int_{\partial \mathcal{O}_{p}}\left(\left(T_{\text {ren }}\right)_{\mathcal{O}_{p}}\right)_{i j} \nu^{i} \nu^{j}\langle Z, \nu\rangle \mathrm{d} \sigma=\lim _{R \rightarrow \infty} \int_{S_{R}}\left(\left(T_{\text {ren }}\right)_{\mathcal{O}_{p}}\right)_{i j} \nu^{i} \nu^{j}\langle Z, \nu\rangle \mathrm{d} \sigma .
$$

To get a decay property of $\left(T_{\text {ren }}\right)_{\mathcal{O}_{p}}$ at infinity, we first recall that

$$
H_{\mathcal{O}}-H_{\text {free }}=-\frac{2}{\pi} \int_{m}^{\infty} \lambda \sqrt{\lambda^{2}-m^{2}}\left(R_{\mathcal{O}, \mathrm{i} \lambda}-R_{\text {free }, \mathrm{i} \lambda}\right) \mathrm{d} \lambda .
$$

For $m \neq 0$, we would have an exponential decay of $e^{-C m \rho(x)}$ for $\left(\breve{H}_{\mathcal{O}}-\breve{H}_{\text {free }}\right)$ $(x, x)$, as explained in the proof of Theorem 3.5. For $m=0$, one could use the estimates of (17) and (18) to obtain that, for $d \geq 2$,

$$
\left|\left(\breve{H}_{\mathcal{O}}-\breve{H}_{\text {free }}\right)(x, x)\right| \leq C \rho^{-2 d+1}(x) .
$$

Moreover, estimate (19) also implies

$$
\left|\left(\Delta\left(\breve{H}_{\mathcal{O}}^{-1}-\breve{H}_{\text {free }}^{-1}\right)\right)(x, x)\right| \leq C \rho^{-2 d+1}(x) .
$$

Applying the above estimates to Theorem 2.4, one concludes that

$$
\left|\left(T_{\text {ren }}\right)_{\mathcal{O}_{p}}\right| \leq C \rho^{-2 d+1}(x) .
$$

This implies $\lim _{R \rightarrow \infty} \int_{S_{R}}\left(\left(T_{\text {ren }}\right)_{\mathcal{O}_{p}}\right)_{i j} \nu^{i} \nu^{j}\langle Y, \nu\rangle \mathrm{d} \sigma=0$.

As $\left(T_{\text {ren }}\right)_{\mathcal{O}}=T_{\text {ren }}$ (see Definition 2.3 and the first two paragraphs of Section 3), this completes the proof.

\section{The Zeta Regularised Energy and the Equivalence of (1) and (3)}

In this section, we assume that $m>0$. In that case it is well known that for $\operatorname{Re}(s)>(d+1) / 2$ the operator

$$
\left(H_{\mathcal{O}}^{-2 s}-H_{\text {free }}^{-2 s}\right)
$$

is trace-class (see for example [53]) and we can therefore define the renormalised zeta functions $\zeta_{\mathcal{O}}(s)$ as

$$
\zeta_{\mathcal{O}}(s)=\operatorname{Tr}\left(H_{\mathcal{O}}^{-2 s}-H_{\text {free }}^{-2 s}\right)=-2 s \int_{0}^{\infty} \lambda\left(\lambda^{2}+m^{2}\right)^{-s-1} \xi(\lambda) \mathrm{d} \lambda
$$

where $\xi(\lambda)$ is the spectral shift function of the problem. The Birman-Krein formula applies to this setting and we have

$$
\xi(\lambda)=-\frac{1}{2 \pi \mathrm{i}} \log \operatorname{det}(S(\lambda))
$$

where $S(\lambda)$ is the stationary scattering matrix of the problem. 
One can also use the Mellin transform to write

$$
\zeta_{\mathcal{O}}(s)=\frac{1}{\Gamma(s)} \int_{0}^{\infty} t^{s-1} h_{\mathcal{O}}(t) \mathrm{d} t
$$

where

$$
\begin{aligned}
h_{\mathcal{O}}(t)=\operatorname{Tr}\left(e^{-t H_{\mathcal{O}}^{2}}-e^{-t H_{\text {free }}^{2}}\right) & =e^{-m^{2} t} \operatorname{Tr}\left(e^{t \Delta_{\mathcal{O}}}-e^{t \Delta_{\text {free }}}\right) \\
& =-2 t e^{-m^{2} t} \int_{0}^{\infty} e^{-\lambda^{2} t} \xi_{\mathcal{O}}(\lambda) \lambda \mathrm{d} \lambda .
\end{aligned}
$$

The following lemma should be well known but we could not find a reference for the precise statement. It is a simple consequence of heat kernel expansions [54-56] and Kac's principle of not feeling the boundary [57,58]. We also refer to $[59,60]$ for more details on obstacle scattering theory and the Birman-Krein formula.

Lemma 6.1. The function $h_{\mathcal{O}}(t)$ is exponentially decaying as $t \rightarrow \infty$ and has a full asymptotic expansion as $t \searrow 0$ of the form

$$
h_{\mathcal{O}}(t) \sim t^{-(d-1) / 2} \sum_{k=0}^{\infty} a_{k} t^{k}, \quad t \rightarrow 0_{+},
$$

where the infinite sum is understood in the sense of asymptotic summation. The coefficients $a_{k}$ are integrals over $\partial \mathcal{O}$ of locally determined quantities expressed in terms of the extrinsic and intrinsic curvature of the boundary and its derivatives. In particular,

$$
a_{0}=-\frac{1}{4}(4 \pi)^{-\frac{d-1}{2}} \operatorname{Vol}_{d-1}(\partial \mathcal{O}) .
$$

Proof. The exponential decay follows immediately from the representation by means of the spectral shift function and $m>0$. Now $h_{\mathcal{O}}(t)$ is the trace of the difference of the two heat operators $e^{-t H_{\mathcal{O}}^{2}}$ and $e^{-t H_{\text {free }}^{2}}$ with integral kernels $K_{\mathcal{O}}(t, x, y)$ and $K_{\text {free }}(t, x, y)$, respectively. Since the difference is trace-class and the integral kernel is smooth we have

$$
h_{\mathcal{O}}(t)=\lim _{R \rightarrow \infty} \int_{\mathcal{E}_{\cap} B_{R}} K_{\mathcal{O}}(t, x, x)-K_{\text {free }}(t, x, x) \mathrm{d} x,
$$

where $B_{R}$ is a ball of radius $R$, i.e. integration is over a large ball of radius $R$ with the obstacles removed. The heat kernel difference satisfies not feeling the boundary estimates. For example, a general finite propagation speed estimate [58] gives

$$
\left|K_{\mathcal{O}}(t, x, x)-K_{\text {free }}(t, x, x)\right| \leq C\left(\rho(x)^{-d}+1\right) t^{-d-\frac{3}{2}} e^{-\frac{\rho(x)^{2}}{t}},
$$

where $\rho(x)=\operatorname{dist}(x, \mathcal{O})$. This shows that

$$
h_{\mathcal{O}}(t)=\int_{\mathcal{E}}\left(K_{\mathcal{O}}(t, x, x)-K_{\text {free }}(t, x, x)\right) \mathrm{d} x .
$$

Let $\mathcal{U} \subset \mathcal{E}$ be an open neighbourhood that contains $\partial \mathcal{E}$, i.e. $\partial \mathcal{E} \subset \mathcal{U} \subset \mathcal{E}$. Then, we have for $|t|<1$ that

$$
h_{\mathcal{O}}(t)=\int_{\mathcal{U}}\left(K_{\mathcal{O}}(t, x, x)-K_{\text {free }}(t, x, x)\right) \mathrm{d} x+O\left(t^{N}\right),
$$


for any $N>0$. This computation is therefore purely local. Using another not feeling the boundary estimate we can replace $K_{\mathcal{O}}(t, x, x)$ in this integral by the Dirichlet heat kernel on the compact manifold obtained by removing $\mathcal{O}$ from the large flat torus. The coefficients are therefore the same as the heat kernel coefficients on a domain with boundary. It is well-known that heat kernel coefficients are determined by local invariants of the jets of the symbols of the operators, i.e. jets of Riemannian metric and second fundamental forms ([56, Lemma 2.6] or [54, Lemma 2.1]). As our interior geometry is Euclidean, only the first heat coefficients corresponding to the interior is nonzero. This first coefficient is the same for both operators and therefore only boundary terms contribute to the expansion. The first non-trivial term is given by $a_{0}=$ $-\frac{1}{4}(4 \pi)^{-\frac{d-1}{2}} \operatorname{Vol}_{d-1}(\partial \mathcal{O})$ (see for example [54, Theorem 1.1]).

Remark 6.2. The general form of the heat expansion for a compact Riemannian manifold $M$ with boundary $\partial M$ is of the form

$$
\operatorname{Tr} e^{t \Delta_{M}} \sim t^{-\frac{d}{2}} \sum_{k=0}^{\infty} b_{k} t^{\frac{k}{2}}, \quad t \rightarrow 0_{+},
$$

where the $b_{2 k}$ are integrals of locally defined quantities over $M$, and the $b_{2 k+1}$ are integrals of locally defined quantities over $\partial M$ which are determined by the boundary conditions. When considering differences heat kernels of Laplace operators with different boundary conditions the $b_{2 k}$ terms cancel and only the terms containing $b_{2 k+1}$ remain.

It follows as usual (for example [61, Sect. 1.12]) that $\zeta_{\mathcal{O}}$ has a meromorphic continuation to the complex plane. If $d$ is odd then there are finitely many poles at $\left\{\frac{d-1}{2}, \frac{d-1}{2}-1, \ldots, 1\right\}$ with residue at $\frac{d-1}{2}-k$ determined by the coefficients $a_{k}$. In this case, the values at non-positive integers are also expressible in terms of $a_{k}$. In case $d$ is even, poles may be located at the points $\left\{\frac{d-1}{2}-k \mid k \in \mathbb{N}_{0}\right\}$.

Definition 6.3. The regularised energy $E_{\text {reg }}$ is then defined

$$
E_{\text {reg }}=\frac{1}{2} \mathrm{FP}_{s=-\frac{1}{2}}\left(\zeta_{\mathcal{O}}(s)\right),
$$

where $\mathrm{FP}_{z=a} f(z)$ denotes the finite part of the meromorphic function $f$ at the point $a$, i.e. the constant term in the Laurent expansion of $f$ about the point $a$.

In particular, in case $d$ is odd we have $E_{\text {reg }}=\frac{1}{2} \zeta_{\mathcal{O}}\left(-\frac{1}{2}\right)$.

We can also define a zeta regularised energy $E_{\text {reg }}^{j}$ for every object $\mathcal{O}_{j}$. Obviously, $E_{\text {reg }}^{j}$ does not depend on the position of $\mathcal{O}_{j}$ in $\mathbb{R}^{d}$ and is also invariant under active rotations of the object. Since the heat coefficients are local quantities the relative zeta function

$$
\zeta_{\text {rel }}(s)=\zeta_{\mathcal{O}}(s)-\sum_{j=1}^{N} \zeta_{\mathcal{O}_{j}}(s)
$$


is an entire function. Since the relative quantities

$$
\left(H_{\mathcal{O}}^{-2 s}-H_{\text {free }}^{-2 s}\right)-\sum_{j=1}^{N}\left(H_{\mathcal{O}_{j}}^{-2 s}-H_{\text {free }}^{-2 s}\right)
$$

are trace-class for $s<0$ we also have that $E_{\text {rel }}=\frac{1}{2} \zeta_{\text {rel }}\left(-\frac{1}{2}\right)$ and therefore

$$
E_{\mathrm{rel}}=E_{\mathrm{reg}}-\sum_{j=1}^{N} E_{\mathrm{reg}}^{j} .
$$

Thus, $E_{\text {rel }}-E_{\text {reg }}$ does not change if the individual objects are translated or rotated.

\section{Proof of Main Theorem}

In this section, we will prove our main theorem (Theorem 1.1) by combining the results we obtained in the previous sections.

Proof of Theorem 1.1. The differentiability of $E_{\text {rel }}(\epsilon)$ follows from Theorems 5.3 and 5.4. As shown in Section 3, we have $\Xi(\lambda)=\log \operatorname{det}\left(Q_{\lambda} \tilde{Q}_{\lambda}^{-1}\right)$ and Theorem 3.7. Recall that equation (21), says

$$
E_{\mathrm{rel}}=\frac{1}{2} \operatorname{Tr} H_{\mathrm{rel}}=\frac{1}{\pi} \int_{m}^{\infty} \frac{\omega}{\sqrt{\omega^{2}-m^{2}}} \Xi(\mathrm{i} \omega) \mathrm{d} \omega .
$$

By substituting $\Xi(\lambda)$, one obtains Eq. (2) of our main theorem. Equation (1) follows immediately from Theorems 5.9 and 5.10. Since the flow $\Phi_{\epsilon}$ described right above Theorem 1.1 is exactly a boundary translation flow (see Definition 5.1), we know from the end of Sect. 6 that $E_{\text {reg }}^{j}$ is constant if the individual objects are translated. Hence, the fact that $E_{\text {rel }}(\epsilon)-E_{\text {reg }}(\epsilon)$ is constant near $\epsilon=0$ for $m>0$ follows.

Open Access. This article is licensed under a Creative Commons Attribution 4.0 International License, which permits use, sharing, adaptation, distribution and reproduction in any medium or format, as long as you give appropriate credit to the original author(s) and the source, provide a link to the Creative Commons licence, and indicate if changes were made. The images or other third party material in this article are included in the article's Creative Commons licence, unless indicated otherwise in a credit line to the material. If material is not included in the article's Creative Commons licence and your intended use is not permitted by statutory regulation or exceeds the permitted use, you will need to obtain permission directly from the copyright holder. To view a copy of this licence, visit http://creativecommons. org/licenses/by/4.0/.

Publisher's Note Springer Nature remains neutral with regard to jurisdictional claims in published maps and institutional affiliations. 


\section{Appendix A: Bounds on the Free Resolvent}

In this appendix, we will give some estimates on the kernels of $\phi G_{\text {free, } \lambda} \phi$ and $\phi G_{\text {free, } \lambda} \chi$, which are denoted by $K_{\lambda}(x, y)$ and $\tilde{K}_{\lambda}(x, y)$, respectively. They are given by

$$
\left\{\begin{array}{l}
K_{\lambda}(x, y)=\frac{\mathrm{i}}{4} \phi(x) \phi(y)\left(\frac{\lambda}{2 \pi|x-y|}\right)^{\frac{d-2}{2}} H_{\frac{d-2}{2}}^{(1)}(\lambda|x-y|), \\
\tilde{K}_{\lambda}(x, y)=\frac{\mathrm{i}}{4} \phi(x) \chi(y)\left(\frac{\lambda}{2 \pi|x-y|}\right)^{\frac{d-2}{2}} H_{\frac{d-2}{2}}^{(1)}(\lambda|x-y|),
\end{array}\right.
$$

where $H_{\nu}^{(1)}$ is the Hankel function. Moreover, we assume $\phi$ has compact support whereas the support of $\chi$ is unbounded with $\operatorname{dist}(\operatorname{supp} \phi, \operatorname{supp} \chi)=\delta>0$. Now we have

$$
\begin{aligned}
\mathcal{K}_{\mu, \nu, p, d}(x ; \lambda) & =\int_{\mathbb{R}^{d}}\left|\phi(x) \phi(y)\left(\frac{\lambda}{2 \pi|x-y|}\right)^{\mu} H_{\nu}^{(1)}(\lambda|x-y|)\right|^{p} \mathrm{~d} y \\
& \leq C \int_{\operatorname{supp}(\phi)}\left|\left(\frac{\lambda}{2 \pi|x-y|}\right)^{\mu} H_{\nu}^{(1)}(\lambda|x-y|)\right|^{p} \mathrm{~d} y \\
& \leq C \int_{\mathbb{S}^{d-1}} \int_{0}^{r_{\phi}}\left|\left(\frac{\lambda}{2 \pi r}\right)^{\mu} H_{\nu}^{(1)}(\lambda r)\right|^{p} r^{d-1} \mathrm{~d} r \mathrm{~d} \omega \\
& \leq C \int_{0}^{r_{\phi}}\left|\left(\frac{\lambda}{r}\right)^{\mu} H_{\nu}^{(1)}(\lambda r)\right|^{p} r^{d-1} \mathrm{~d} r .
\end{aligned}
$$

Similarly,

$$
\tilde{\mathcal{K}}_{\mu, \nu, p, d}(x ; \lambda) \leq C \int_{\delta}^{\infty}\left|\left(\frac{\lambda}{r}\right)^{\mu} H_{\nu}^{(1)}(\lambda r)\right|^{p} r^{d-1} \mathrm{~d} r
$$

Recall that Hankel functions have the following asymptotic

$$
\begin{array}{ll}
\text { For }|z|<r_{0}, & \left|H_{\nu}^{(1)}(z)\right| \leq C_{r_{0}, \nu} \begin{cases}|z|^{-\nu} & \text { if } \nu>0, \\
|\log (z)| & \text { if } \nu=0 .\end{cases} \\
\text { For }|z| \geq r_{0}, & \left|H_{\nu}^{(1)}(z)\right| \leq C_{r_{0}, \nu}|z|^{-\frac{1}{2}} e^{-\operatorname{Im} z} .
\end{array}
$$

Therefore, for small $\lambda$ such that $r_{\phi}|\lambda|<r_{0}$, we have

$$
\int_{0}^{r_{\phi}}\left|\left(\frac{\lambda}{r}\right)^{\mu} H_{\nu}^{(1)}(\lambda r)\right|^{p} r^{d-1} \mathrm{~d} r \leq \begin{cases}C\left|\lambda^{\mu} \log (\lambda)\right|^{p} \int_{0}^{r_{\phi}} r^{d-\mu p-1}|\log r|^{p} \mathrm{~d} r & \text { for } \nu=0, \\ C|\lambda|^{(\mu-\nu) p} \int_{0}^{r_{\phi}} r^{d-1-(\mu+\nu) p} \mathrm{~d} r & \text { for } \nu \neq 0 .\end{cases}
$$


For large $\lambda$ such that $r_{\phi}|\lambda| \geq r_{0}$, we have

$$
\begin{aligned}
& \int_{0}^{r_{\phi}}\left|\left(\frac{\lambda}{r}\right)^{\mu} H_{\nu}^{(1)}(\lambda r)\right|^{p} r^{d-1} \mathrm{~d} r \\
& =|\lambda|^{2 \mu p-d} \int_{0}^{r_{\phi}|\lambda|}\left|H_{\nu}^{(1)}\left(\frac{\lambda}{|\lambda|} r\right)\right|^{p} r^{d-\mu p-1} \mathrm{~d} r \\
& =|\lambda|^{2 \mu p-d}\left(\int_{0}^{r_{0}}\left|H_{\nu}^{(1)}\left(\frac{\lambda}{|\lambda|} r\right)\right|^{p} r^{d-\mu p-1} \mathrm{~d} r+\int_{r_{0}}^{r_{\phi}|\lambda|}\left|H_{\nu}^{(1)}\left(\frac{\lambda}{|\lambda|} r\right)\right|^{p} r^{d-\mu p-1} \mathrm{~d} r\right) .
\end{aligned}
$$

The above terms can be bounded by using the asymptotic of Hankel functions in Eqs. (49) and (50).

$$
\begin{cases}|\lambda|^{2 \mu p-d}\left(\int_{0}^{r_{0}}\left|\log \left(\frac{\lambda}{|\lambda|} r\right)\right|^{p} r^{d-\mu p-1} \mathrm{~d} r+\int_{r_{0}}^{r_{\phi}|\lambda|} r^{-\frac{1}{2}} e^{-r \frac{\operatorname{Im} \lambda}{|\lambda|}} r^{d-\mu p-1} \mathrm{~d} r\right) & \text { for } \nu=0, \\ |\lambda|^{2 \mu p-d}\left(\int_{0}^{r_{0}} r^{d-(\mu+\nu) p-1} \mathrm{~d} r+\int_{r_{0}}^{r_{\phi}|\lambda|} r^{-\frac{1}{2}} e^{-r \frac{\operatorname{Im} \lambda}{|\lambda|}} r^{d-\mu p-1} \mathrm{~d} r\right) & \text { for } \nu \neq 0 .\end{cases}
$$

Replacing $r_{\phi}|\lambda|$ by $\infty$ in the integral, we obtain

$$
\mathcal{K}_{\mu, \nu, p, d}(x ; \lambda) \leq C_{r_{0}, \mu, \nu, p, d}|\lambda|^{2 \mu p-d} \text { for large }|\lambda| .
$$

Combining with the asymptotic for small $|\lambda|$, we have

$$
\mathcal{K}_{\mu, \nu, p, d}(x ; \lambda) \leq \begin{cases}C_{r_{0}, \mu, p, d} \rho_{p, \mu p ; 2 \mu p-d}(\operatorname{Im} \lambda) & \text { for } \nu=0 \\ C_{r_{0}, \mu, \nu, p, d} \rho_{0,(\mu-\nu) p ; 2 \mu p-d}(\operatorname{Im} \lambda) & \text { for } \nu \neq 0 .\end{cases}
$$

For $\tilde{\mathcal{K}}_{\mu, \nu, p, d}$, we have

$$
\tilde{\mathcal{K}}_{\mu, \nu, p, d}(x ; \lambda) \leq C \int_{\delta}^{\infty}\left|\left(\frac{\lambda}{r}\right)^{\mu} H_{\nu}^{(1)}(\lambda r)\right|^{p} r^{d-1} \mathrm{~d} r .
$$

For small $\lambda$ such that $\delta|\lambda|<r_{0}$, we also have

$$
\begin{aligned}
& \int_{\delta}^{\infty}\left|\left(\frac{\lambda}{r}\right)^{\mu} H_{\nu}^{(1)}(\lambda r)\right|^{p} r^{d-1} \mathrm{~d} r \\
& =|\lambda|^{2 \mu p-d} \int_{\delta|\lambda|}^{\infty}\left|H_{\nu}^{(1)}\left(\frac{\lambda}{|\lambda|} r\right)\right|^{p} r^{d-\mu p-1} \mathrm{~d} r \\
& =|\lambda|^{2 \mu p-d}\left(\int_{\delta|\lambda|}^{r_{0}}\left|H_{\nu}^{(1)}\left(\frac{\lambda}{|\lambda|} r\right)\right|^{p} r^{d-\mu p-1} \mathrm{~d} r+\int_{r_{0}}^{\infty}\left|H_{\nu}^{(1)}\left(\frac{\lambda}{|\lambda|} r\right)\right|^{p} r^{d-\mu p-1} \mathrm{~d} r\right) .
\end{aligned}
$$

This can be bounded by

$$
\begin{cases}|\lambda|^{2 \mu p-d}\left(\int_{\delta|\lambda|}^{r_{0}}\left|\log \left(\frac{\lambda}{|\lambda|} r\right)\right|^{p} r^{d-\mu p-1} \mathrm{~d} r+\int_{r_{0}}^{\infty} r^{-\frac{1}{2}} e^{-r \frac{\operatorname{Im} \lambda}{|\lambda|}} r^{d-\mu p-1} \mathrm{~d} r\right) & \text { for } \nu=0, \\ |\lambda|^{2 \mu p-d}\left(\int_{\delta|\lambda|}^{r_{0}} r^{d-(\mu+\nu) p-1} \mathrm{~d} r+\int_{r_{0}}^{\infty} r^{-\frac{1}{2}} e^{-r \frac{\operatorname{Im} \lambda}{|\lambda|}} r^{d-\mu p-1} \mathrm{~d} r\right) & \text { for } \nu \neq 0 .\end{cases}
$$

Replacing $\delta|\lambda|$ by 0 , one has

$$
\tilde{\mathcal{K}}_{\mu, \nu, p}(x ; \lambda) \leq C_{\mu, \nu, p, d}|\lambda|^{2 \mu p-d} \quad \text { for small }|\lambda| .
$$


For large $\lambda$ such that $\delta|\lambda| \geq r_{0}$, we have

$$
\begin{aligned}
\int_{\delta}^{\infty}\left|\left(\frac{\lambda}{r}\right)^{\mu} H_{\nu}^{(1)}(\lambda r)\right|^{p} r^{d-1} \mathrm{~d} r & \leq\left.\left. C \int_{\delta}^{\infty}\left|\left(\frac{\lambda}{r}\right)^{\mu}\right| \lambda r\right|^{-\frac{1}{2}} e^{-r \operatorname{Im} \lambda}\right|^{p} r^{d-1} \mathrm{~d} r \\
& \leq C|\lambda|^{\mu p-\frac{p}{2}} \int_{\delta}^{\infty} r^{d-\mu p-\frac{p}{2}-1} e^{-r \operatorname{Im} \lambda} \mathrm{d} r \\
& \leq C|\lambda|^{\mu p-\frac{p}{2}} e^{-\delta \operatorname{Im} \lambda} \int_{0}^{\infty}(r+\delta)^{d-\mu p-\frac{p}{2}-1} e^{-r \operatorname{Im} \lambda} \mathrm{d} r \\
& \leq C_{\delta, r_{0}, \mu, p, d} e^{-\delta \operatorname{Im} \lambda} .
\end{aligned}
$$

This implies

$$
\tilde{\mathcal{K}}_{\mu, \nu, p, d}(x ; \lambda) \leq C_{r_{0}, \mu, \nu, p, d} \rho_{0,2 \mu p-d ; 0}(\operatorname{Im} \lambda) e^{-\delta \operatorname{Im} \lambda} .
$$

By the Schur test and Eq. (51), we have

$$
\left\|\phi G_{\text {free }, \lambda} \phi\right\|_{L^{2} \rightarrow L^{2}} \leq \sup _{x \in \operatorname{supp}(\phi)} \mathcal{K}_{\frac{d-2}{2}, \frac{d-2}{2}, 1, d}(x ; \lambda) \leq C_{r_{0}, \phi} \begin{cases}\rho_{1,0 ;-2}(\operatorname{Im} \lambda) & \text { for } d=2, \\ \rho_{0,0 ;-2}(\operatorname{Im} \lambda) & \text { for } d \neq 2 .\end{cases}
$$

Using the recurrence relations of Hankel functions $\left(H_{\nu}^{(1)}\right)^{\prime}(z)=-H_{\nu+1}^{(1)}(z)+\frac{\nu}{z} H_{\nu}^{(1)}(z)$ and similar calculations, one has

$$
\left\|\nabla_{i} \circ\left(\phi G_{\text {free }, \lambda} \phi\right)\right\|_{L^{2} \rightarrow L^{2}} \leq C_{r_{0}, \phi} \begin{cases}\rho_{1,0 ;-1}(\operatorname{Im} \lambda) & \text { for } d=2, \\ \rho_{0,0 ;-1}(\operatorname{Im} \lambda) & \text { for } d \neq 2 .\end{cases}
$$

This is the same bound for $\left\|\phi G_{\text {free, } \lambda} \phi\right\|_{L^{2} \rightarrow H^{1}}$. To get the bound for $\left\|\phi G_{\text {free }, \lambda} \phi\right\|_{L^{2} \rightarrow H^{2}}$, we recall that $\left(-\Delta-\lambda^{2}\right) G_{\text {free }, \lambda}=I$, hence

$$
\begin{aligned}
\left\|\phi G_{\text {free }, \lambda} \phi\right\|_{L^{2} \rightarrow H^{2}} & \leq C\left\|\Delta \phi G_{\text {free }, \lambda} \phi\right\|_{L^{2} \rightarrow L^{2}}+C\left\|\phi G_{\text {free }, \lambda} \phi\right\|_{L^{2} \rightarrow L 2} \\
& \leq C\left(1+|\lambda|^{2}\right)\left\|\phi G_{\text {free }, \lambda} \phi\right\|_{L^{2} \rightarrow L^{2}}+C\left\|\tilde{\phi} G_{\text {free }, \lambda} \tilde{\phi}\right\|_{L^{2} \rightarrow H^{1}},
\end{aligned}
$$

where $\tilde{\phi}=1$ near $\operatorname{supp} \phi$ and $\operatorname{diam}(\operatorname{supp} \tilde{\phi})$ is bounded. Combining with the estimates on $\left\|\phi G_{\text {free }, \lambda} \phi\right\|_{L^{2} \rightarrow H^{1}}$ and $\left\|\phi G_{\text {free }, \lambda} \phi\right\|_{L^{2} \rightarrow L^{2}}$, we have

$$
\left\|\phi G_{\text {free }, \lambda} \phi\right\|_{L^{2} \rightarrow H^{2}} \leq C_{r_{0}, \phi} \begin{cases}\rho_{1,0 ; 0}(\operatorname{Im} \lambda) & \text { for } d=2, \\ 1 & \text { for } d \neq 2 .\end{cases}
$$

For the operator $\chi G_{\text {free, } \lambda} \phi$, we obtain from Eq. (52) that

$$
\begin{aligned}
\left\|\chi G_{\text {free }, \lambda} \phi\right\|_{H S\left(H^{s}(\partial \mathcal{O}) \rightarrow L^{2}\left(\mathbb{R}^{d}\right)\right)}^{2} & \leq \sup _{x \in \operatorname{supp}(\phi)} \tilde{\mathcal{K}}_{\frac{d-2}{2}, \frac{d-2}{2}, 2, d}(x ; \lambda) \\
& \leq C_{r_{0}, \phi, \chi} \rho_{0, d-4 ; 0}(\operatorname{Im} \lambda) e^{-\delta \operatorname{Im} \lambda},
\end{aligned}
$$

which gives the verification of the estimate (24).

\section{Appendix B: The Method Illustrated for the 1-D Casimir Effect}

In this appendix, we illustrate Theorem 5.9 in its simplest form, i.e. for the case of the one-dimensional Casimir effect. This will also illustrate the advantages 
of the relative framework. Let $a_{1}<b_{1}<a_{2}<b_{2}$, where $\mathcal{O}_{1}=\left(a_{1}, b_{1}\right)$ and $\mathcal{O}_{2}=\left(a_{2}, b_{2}\right)$ are the obstacles. Then, we have

$$
\begin{array}{r}
G_{\mathcal{O}}=\left\{\begin{array}{lr}
G_{\left(-\infty, a_{1}\right)} & x, y<a_{1} \\
G_{\left(a_{1}, b_{1}\right)} & a_{1}<x, y<b_{1} \\
G_{\left(b_{1}, a_{2}\right)} & b_{1}<x, y<a_{2}, \\
G_{\left(a_{2}, b_{2}\right)} & a_{2}<x, y<b_{2} \\
G_{\left(b_{2},+\infty\right)} & x, y>b_{2}
\end{array}\right. \\
G_{\mathcal{O}_{1}}=\left\{\begin{array}{lr}
G_{\left(-\infty, a_{1}\right)} & x, y<a_{1} \\
G_{\left(a_{1}, b_{1}\right)} & a_{1}<x, y<b_{1}, \\
G_{\left(b_{1},+\infty\right)} & x, y>b_{1}
\end{array}\right. \\
G_{\mathcal{O}_{2}}=\left\{\begin{array}{lr}
G_{\left(-\infty, a_{2}\right)} & x, y<a_{2} \\
G_{\left(a_{2}, b_{2}\right)} & a_{2}<x, y<b_{2} . \\
G_{\left(b_{2},+\infty\right)} & x, y>b_{2}
\end{array}\right.
\end{array}
$$

Then, $G_{\text {rel }}=G_{\mathcal{O}}-G_{\mathcal{O}_{1}}-G_{\mathcal{O}_{2}}+G_{\text {free }}$ is given by

$$
G_{\text {rel }}=\left\{\begin{array}{lr}
G_{\text {free }}-G_{\left(-\infty, a_{2}\right)} & x, y<b_{1} \\
G_{\left(b_{1}, a_{2}\right)}-G_{\left(b_{1},+\infty\right)}-G_{\left(-\infty, a_{2}\right)}+G_{\text {free }} & b_{1}<x, y<a_{2} . \\
G_{\text {free }}-G_{\left(b_{1},+\infty\right)} & x, y>a_{2}
\end{array}\right.
$$

In particular,

$$
\begin{aligned}
& G_{\text {free }}\left(x, y ; k^{2}\right)=-\frac{e^{\mathrm{i} k|x-y|}}{2 \mathrm{i} k}= \begin{cases}-\frac{e^{-\mathrm{i} k(x-y)}}{2 \mathrm{i} k} & x<y \\
-\frac{e^{-\mathrm{i} k(y-x)}}{2 \mathrm{i} k} & x>y\end{cases} \\
& G_{(b,+\infty)}\left(x, y ; k^{2}\right)=-\frac{e^{\mathrm{i} k|x-y|}-e^{\mathrm{i} k|x+y-2 b|}}{2 \mathrm{i} k}=\left\{\begin{array}{ll}
\frac{e^{\mathrm{i} k(y-b)} \sin (k(x-b))}{k} & x<y \\
\frac{e^{\mathrm{i} k(x-b)} \sin (k(y-b))}{k} & x>y
\end{array},\right. \\
& G_{(-\infty, a)}\left(x, y ; k^{2}\right)=-\frac{e^{\mathrm{i} k|x-y|}-e^{\mathrm{i} k|x+y-2 a|}}{2 \mathrm{i} k}= \begin{cases}\frac{e^{-\mathrm{i} k(x-a)} \sin (k(a-y))}{k} & x<y \\
\frac{e^{-\mathrm{i} k(y-a)} \sin (k(a-x))}{k} & x>y\end{cases} \\
& G_{(a, b)}\left(x, y ; k^{2}\right)=\frac{\cos (k(x+y-b-a))-\cos (k(b-a-|x-y|))}{2 k \sin (k(b-a))} \\
& = \begin{cases}\frac{\sin (k(x-a)) \sin (k(b-y))}{k \sin (k(b-a))} \quad x<y \\
\frac{\sin (k(y-a)) \sin (k(b-x))}{k \sin (k(b-a))} \quad x>y\end{cases}
\end{aligned}
$$

For $x, y>b$, we have

$$
\left[G_{\text {free }}-G_{(b,+\infty)}\right]\left(x, y ; k^{2}\right)=-\frac{e^{\mathrm{i} k|x+y-2 b|}}{2 \mathrm{i} k},
$$


which implies

$$
\begin{aligned}
{\left[\Delta_{\text {free }}^{\frac{1}{2}}-\Delta_{(b,+\infty)}^{\frac{1}{2}}\right](x, y) } & =\frac{\mathrm{i}}{\pi} \int_{\tilde{\Gamma}} k \sqrt{k^{2}}\left[G_{\text {free }}-G_{(b,+\infty)}\right]\left(x, y ; k^{2}\right) \mathrm{d} k \\
& =\frac{\mathrm{i}}{\pi} \int_{\tilde{\Gamma}} k \sqrt{k^{2}}\left(-\frac{e^{\mathrm{i} k|x+y-2 b|}}{2 \mathrm{i} k}\right) \mathrm{d} k \\
& =-\frac{\mathrm{i}}{\pi} \int_{-\tilde{\Gamma}} k \sqrt{k^{2}}\left(-\frac{e^{\mathrm{i} k|x+y-2 b|}}{2 \mathrm{i} k}\right) \mathrm{d} k \\
& =-\frac{2 \mathrm{i}}{\pi} \int_{0}^{\infty}(\mathrm{i} k)^{2}\left(-\frac{e^{\mathrm{i}(\mathrm{i} k)|x+y-2 b|}}{2 \mathrm{i}(\mathrm{i} k)}\right) \mathrm{di} k \\
& =-\frac{1}{\pi(x+y-2 b)^{2}} .
\end{aligned}
$$

The same calculation yields for $x, y<b$. That is

$$
\left[\Delta_{\text {free }}^{\frac{1}{2}}-\Delta_{(b,+\infty)}^{\frac{1}{2}}\right](x, y)=\left[\Delta_{\text {free }}^{\frac{1}{2}}-\Delta_{(-\infty, b)}^{\frac{1}{2}}\right](x, y)=-\frac{1}{\pi(x+y-2 b)^{2}} \text {. }
$$

When restricting to the diagonal, we have

$$
\left[\Delta_{\text {free }}^{\frac{1}{2}}-\Delta_{(b,+\infty)}^{\frac{1}{2}}\right](x, x)=\left[\Delta_{\text {free }}^{\frac{1}{2}}-\Delta_{(-\infty, b)}^{\frac{1}{2}}\right](x, x)=-\frac{1}{4 \pi(x-b)^{2}} .
$$

Now for $a<x, y<b$, we have

$$
\begin{aligned}
{\left[G_{(a, b)}-G_{\text {free }}\right]\left(x, y ; k^{2}\right)=} & \frac{\cos (k(b-a-|x-y|))-\cos (k(x+y-b-a))}{2 k \sin (k(b-a))} \\
& +\frac{e^{\mathrm{i} k|x-y|}}{2 \mathrm{i} k},
\end{aligned}
$$

which implies

$$
\begin{aligned}
{\left[\Delta_{(a, b)}^{\frac{1}{2}}-\Delta_{\text {free }}^{\frac{1}{2}}\right](x, y) } & =\frac{\mathrm{i}}{\pi} \int_{\tilde{\Gamma}} k \sqrt{k^{2}}\left[G_{(a, b)}-G_{\text {free }}\right]\left(x, y ; k^{2}\right) \mathrm{d} k \\
& =-\frac{\mathrm{i}}{\pi} \int_{-\tilde{\Gamma}} k \sqrt{k^{2}}\left[G_{(a, b)}-G_{\text {free }}\right]\left(x, y ; k^{2}\right) \mathrm{d} k \\
& =-\frac{2 \mathrm{i}}{\pi} \int_{0}^{\infty}(\mathrm{i} k)^{2}\left[G_{(a, b)}-G_{\text {free }}\right]\left(x, y ;(i k)^{2}\right) \mathrm{di} k \\
& =-\frac{2}{\pi} \int_{0}^{\infty} k^{2}\left[G_{(a, b)}-G_{\text {free }}\right]\left(x, y ;(i k)^{2}\right) \mathrm{d} k
\end{aligned}
$$

Note that

$$
\int_{0}^{\infty} \frac{k \cosh (a k)}{\sinh (b k)} \mathrm{d} k=\frac{\pi^{2}}{4 b^{2}} \sec ^{2}\left(\frac{a \pi}{2 b}\right) \quad \text { for } \quad a<b
$$

and

$$
\begin{aligned}
{\left[G_{(a, b)}-G_{\text {free }}\right]\left(x, y ;(\mathrm{i} k)^{2}\right)=} & \frac{\cosh (k(b-a-|x-y|))-\cosh (k(x+y-b-a))}{2 k \sinh (k(b-a))} \\
& -\frac{e^{-k|x-y|}}{2 k}
\end{aligned}
$$


implies

$$
\begin{aligned}
& {\left[\Delta_{(a, b)}^{\frac{1}{2}}-\Delta_{\text {free }}^{\frac{1}{2}}\right](x, y)} \\
& \quad=-\frac{2}{\pi} \int_{0}^{\infty} k^{2}\left[G_{(a, b)}-G_{\text {free }}\right]\left(x, y ;(\mathrm{i} k)^{2}\right) \mathrm{d} k \\
& \quad=-\frac{\pi}{4(b-a)^{2}}\left[\csc ^{2}\left(\frac{|x-y| \pi}{2(b-a)}\right)-\csc ^{2}\left(\frac{(x+y-2 b) \pi}{2(b-a)}\right)\right]+\frac{1}{\pi(x-y)^{2}} .
\end{aligned}
$$

When restricting to the diagonal, we have

$$
\left[\Delta_{(a, b)}^{\frac{1}{2}}-\Delta_{\text {free }}^{\frac{1}{2}}\right](x, x)=-\frac{\pi}{12(b-a)^{2}}+\frac{\pi}{4(b-a)^{2}} \csc ^{2}\left(\frac{(x-b) \pi}{b-a}\right) .
$$

Equation (53) gives

$$
\frac{1}{2} H_{\text {rel }}(x)=\left\{\begin{array}{lr}
\frac{1}{2}\left[\Delta_{\text {free }}^{\frac{1}{2}}-\Delta_{\left(-\infty, a_{2}\right)}^{\frac{1}{2}}\right](x, x) & x<b_{1} \\
\frac{1}{2}\left[\Delta_{\left(b_{1}, a_{2}\right)}^{\frac{1}{2}}-\Delta_{\left(b_{1}, \infty\right)}^{\frac{1}{2}}-\Delta_{\left(-\infty, a_{2}\right)}^{\frac{1}{2}}+\Delta_{\text {free }}^{\frac{1}{2}}\right](x, x) & b_{1}<x<a_{2} . \\
\frac{1}{2}\left[\Delta_{\text {free }}^{\frac{1}{2}}-\Delta_{\left(-\infty, b_{1}\right)}^{\frac{1}{2}}\right](x, x) & x>a_{2}
\end{array}\right.
$$

From Eqs. (54) and (55), we have

$$
\begin{aligned}
& \frac{1}{2} H_{\mathrm{rel}}(x) \\
& =\left\{\begin{array}{lr}
-\frac{1}{8 \pi\left(x-a_{2}\right)^{2}} & x<b_{1} \\
\frac{\pi}{8\left(a_{2}-b_{1}\right)^{2}} \csc ^{2}\left(\frac{\left(x-a_{2}\right) \pi}{a_{2}-b_{1}}\right)-\frac{\pi}{24\left(a_{2}-b_{1}\right)^{2}}-\frac{1}{8 \pi\left(x-a_{2}\right)^{2}}-\frac{1}{8 \pi\left(x-b_{1}\right)^{2}} & b_{1}<x<a_{2} . \\
-\frac{1}{8 \pi\left(x-b_{1}\right)^{2}} & x>a_{2}
\end{array}\right.
\end{aligned}
$$

This equation shows that $H_{\mathrm{rel}}(x)$ is continuous, which is consistent with the claim in the proof of Theorem 5.8. Integrating over $\mathbb{R}$, we have

$$
\frac{1}{2} \operatorname{Tr}_{\mathbb{R}}\left(H_{\mathrm{rel}}\right)=-\frac{1}{8 \pi\left(a_{2}-b_{1}\right)}+\frac{6-\pi^{2}}{24 \pi\left(a_{2}-b_{1}\right)}-\frac{1}{8 \pi\left(a_{2}-b_{1}\right)}=-\frac{\pi}{24\left(a_{2}-b_{1}\right)}
$$

Similarly, one has the renormalised counterpart of $H_{\text {rel }}(x)$, which is given by $\left(H_{\mathcal{O}}\right)_{\text {ren }}=\left.\left[\Delta_{\mathcal{O}}^{\frac{1}{2}}-\Delta_{\text {free }}^{\frac{1}{2}}\right]\right|_{\Delta}$. Note that this only corresponds to the first term in 
$T_{00}$ of $(5)$, i.e. $\left.\frac{1}{2}\left(H-H_{\text {free }}\right)\right|_{\Delta}$. It is given by

$$
\frac{1}{2} H_{\mathrm{ren}}(x)=\left\{\begin{array}{lc}
\frac{1}{8 \pi\left(x-a_{1}\right)^{2}} & x<a_{1} \\
\frac{\pi}{8\left(b_{1}-a_{1}\right)^{2}} \csc ^{2}\left(\frac{\left(x-b_{1}\right) \pi}{b_{1}-a_{1}}\right)-\frac{\pi}{24\left(a_{2}-b_{1}\right)^{2}} & a_{1}<x<b_{1} \\
\frac{\pi}{8\left(a_{2}-b_{1}\right)^{2}} \csc ^{2}\left(\frac{\left(x-a_{2}\right) \pi}{a_{2}-b_{1}}\right)-\frac{\pi}{24\left(a_{2}-b_{1}\right)^{2}} & b_{1}<x<a_{2} . \\
\frac{\pi}{8\left(b_{2}-a_{2}\right)^{2}} \csc ^{2}\left(\frac{\left(x-b_{2}\right) \pi}{b_{2}-a_{2}}\right)-\frac{\pi}{24\left(b_{2}-a_{2}\right)^{2}} & a_{2}<x<b_{2} \\
\frac{1}{8 \pi\left(x-b_{2}\right)^{2}} & x>b_{2}
\end{array}\right.
$$

It is easy to see that $H_{\text {ren }}$ is not integrable. Therefore, some regularisation schemes would be needed at this point. One way is by heat kernel regularisation (see, for instance, [19]). However, this only resolves the non-integrability problem of the first term of $T_{00}$. We also need to integrate the term $\frac{1}{8} \Delta\left[\left(H^{-1}-\right.\right.$ $\left.\left.H_{\text {free }}^{-1}\right)\left.\right|_{\Delta}\right]$ in Eq. (5) over $\mathbb{R}$. This is also ill-defined, as it is not integrable. We will see that these problems disappear when we work in the relative setting.

Restricting Eq. (53) to the diagonal and then taking the action of Laplacian, we have

$$
\begin{aligned}
& \Delta\left(G_{\text {rel }}\left(x, x ;(\mathrm{i} k)^{2}\right)\right)= \\
& \left\{\begin{array}{lr}
2 k e^{-2 k\left(a_{2}-x\right)} & x<b_{1} \\
-2 k \frac{\cosh \left(k\left(2 x-a_{2}-b_{1}\right)\right)}{\sinh \left(k\left(a_{2}-b_{1}\right)\right)}+2 k\left[e^{-2 k\left(a_{2}-x\right)}+e^{-2 k\left(x-b_{1}\right)}\right] & b_{1}<x<a_{2} . \\
2 k e^{-2 k\left(x-b_{1}\right)} & x>a_{2}
\end{array}\right.
\end{aligned}
$$

Integrating spectral variable $k$ along $\tilde{\Gamma}$ and then over the space variable $x$, we have

$$
\int_{\mathbb{R}} \Delta\left(\left.H_{\mathrm{rel}}^{-1}\right|_{\Delta}\right) \mathrm{d} x=0
$$

hence

$$
E_{\mathrm{rel}}=\frac{1}{2} \operatorname{Tr}_{\mathbb{R}}\left(H_{\mathrm{rel}}\right)+\int_{\mathbb{R}} \frac{1}{8} \Delta\left(\left.H_{\mathrm{rel}}^{-1}\right|_{\Delta}\right) \mathrm{d} x=-\frac{\pi}{24\left(a_{2}-b_{1}\right)} .
$$

Note that using heat kernel regularisation, one would also obtain $E_{\text {rel }}$, see [19]. Equations (56) and (58) agree with Theorem 3.7. Note that equation (57) also shows that

$$
\begin{aligned}
\int_{\mathbb{R}} \Delta\left[\left.\left(H_{\mathcal{O}}^{-1}-H_{\text {free }}^{-1}\right)\right|_{\Delta}\right] \mathrm{d} x^{"}= & " \int_{\mathbb{R}} \Delta\left[\left.\left(H_{\mathcal{O}_{1}}^{-1}-H_{\text {free }}^{-1}\right)\right|_{\Delta}\right] \mathrm{d} x+\int_{\mathbb{R}} \Delta\left[\left(H_{\mathcal{O}_{2}}^{-1}\right.\right. \\
& \left.\left.-H_{\text {free }}^{-1}\right)\left.\right|_{\Delta}\right] \mathrm{d} x
\end{aligned}
$$


where all the three terms are ill-defined as they are not integrable. For instance, $\Delta\left[\left.\left(H_{\mathcal{O}_{1}}^{-1}-H_{\text {free }}^{-1}\right)\right|_{\Delta}\right](x)$ has $\frac{1}{\left(a_{1}-x\right)^{2}}$ singularity when approaching $x=a_{1}$. This justifies Remark 2.5.

Now let $X$ be a smooth vector field that generates a movement of (right) obstacle 2 to the right with a constant speed $v$. Moreover, $X$ is zero around (left) obstacle 1 . In other words, we move the obstacle 2 to the right by $v \epsilon$ and keep the obstacle 1 stationary. Now the variation of the relative energy is given by $\delta_{X} E=v \cdot \partial_{a_{2}} E$. The left-hand side of Eq. in Theorem 5.8 becomes

$$
\delta_{X} E_{\text {rel }}=\frac{v \pi}{24\left(a_{2}-b_{1}\right)^{2}} .
$$

Now the identity (48) used in the proof of Theorem 5.9 says

$$
\frac{1}{2} \operatorname{Tr}\left[\delta_{X} H_{\mathrm{rel}}\right]=-\left.\frac{v}{4} \sum_{p=1}^{N} \int_{\partial \mathcal{O}_{p}}\left[\partial_{\nu} \partial_{\nu}^{\prime}\left(H_{\mathcal{O}}^{-1}-H_{\mathcal{O}_{p}}^{-1}\right)\right]\right|_{\Delta} \mathrm{d} x .
$$

It becomes

$$
\begin{aligned}
\frac{1}{2} \operatorname{Tr}\left[\delta_{X} \Delta_{\text {rel }}^{\frac{1}{2}}\right] & =-\frac{v}{4}\left[\left[\partial_{\nu} \partial_{\nu}^{\prime}\left(\Delta_{\mathcal{O}}^{-\frac{1}{2}}-\Delta_{\mathcal{O}_{2}}^{-\frac{1}{2}}\right)\right]\left(a_{2}, a_{2}\right)-\left[\partial_{\nu} \partial_{\nu}^{\prime}\left(\Delta_{\mathcal{O}}^{-\frac{1}{2}}-\Delta_{\mathcal{O}_{2}}^{-\frac{1}{2}}\right)\right]\left(b_{2}, b_{2}\right)\right] \\
& =-\frac{v}{4}\left[\partial_{\nu} \partial_{\nu}^{\prime}\left(\Delta_{\mathcal{O}}^{-\frac{1}{2}}-\Delta_{\mathcal{O}_{2}}^{-\frac{1}{2}}\right)\right]\left(a_{2}, a_{2}\right) .
\end{aligned}
$$

Note that

$$
\partial_{x} \partial_{y}\left(G_{(a, b)}-G_{(-\infty, b)}\right)\left(b, b ; k^{2}\right)=-\mathrm{i} k-k \cot (k(b-a))
$$

therefore

$$
\begin{aligned}
& {\left[\partial_{\nu} \partial_{\nu}^{\prime}\left(\Delta_{\mathcal{O}}^{-\frac{1}{2}}-\Delta_{\mathcal{O}_{2}}^{-\frac{1}{2}}\right)\right]\left(a_{2}, a_{2}\right)=\frac{\mathrm{i}}{\pi} \int_{\tilde{\Gamma}} \frac{k}{\sqrt{k^{2}}} \partial_{x} \partial_{y}\left[G_{\left(b_{1}, a_{2}\right)}-G_{\left(-\infty, a_{2}\right)}\right]\left(a_{2}, a_{2} ; k^{2}\right) \mathrm{d} k} \\
& \quad=-\frac{\mathrm{i}}{\pi} \int_{-\tilde{\Gamma}} \frac{k}{\sqrt{k^{2}}} \partial_{x} \partial_{y}\left[G_{\left(b_{1}, a_{2}\right)}-G_{\left(-\infty, a_{2}\right)}\right]\left(a_{2}, a_{2} ; k^{2}\right) \mathrm{d} k \\
& \quad=-\frac{2 \mathrm{i}}{\pi} \int_{0}^{\infty} \partial_{x} \partial_{y}\left[G_{\left(b_{1}, a_{2}\right)}-G_{\left(-\infty, a_{2}\right)}\right]\left(a_{2}, a_{2} ;(\mathrm{i} k)^{2}\right) \mathrm{id} k \\
& \quad=\frac{2}{\pi} \int_{0}^{\infty}\left(k-k \operatorname{coth}\left(k\left(a_{2}-b_{1}\right)\right)\right) \mathrm{d} k \\
& \quad=-\frac{\pi}{6\left(a_{2}-b_{1}\right)^{2}} .
\end{aligned}
$$

Combining Eqs. (58), (60) and (61), we have verified the identity (48) in onedimensional cases. Moreover, Eqs. (59), (60) and (61) are consistent with Theorems 5.8 and 5.9 .

\section{References}

[1] Casimir, H.B.G.: On the attraction between two perfectly conducting plates. Indag. Math. 10, 261-263 (1948)

[2] Sparnaay, M.J.: Measurements of attractive forces between flat plates. Physica 24(6), 751-764 (1958)

[3] Bressi, G., Carugno, G., Onofrio, R., Ruoso, G.: Measurement of the Casimir force between parallel metallic surfaces. Phys. Rev. Lett. 88, 041804 (2002) 
[4] Chan, H.B., Aksyuk, V.A., Kleiman, R.N., Bishop, D.J., Capasso, F.: Quantum mechanical actuation of microelectromechanical systems by the Casimir force. Science 291(5510), 1941-1944 (2001)

[5] Ederth, T.: Template-stripped gold surfaces with $0.4 \mathrm{~nm}$ rms roughness suitable for force measurements: application to the Casimir force in the 20-100 nm range. Phys. Rev. A 62, 062104 (2000)

[6] Lamoreaux, S.K.: Demonstration of the Casimir force in the 0.6 to $6 \mu \mathrm{m}$ range. Phys. Rev. Lett. 78(1), 5 (1997)

[7] Mohideen, U., Roy, A.: Precision measurement of the Casimir force from 0.1 to $0.9 \mu \mathrm{m}$. Phys. Rev. Lett. 81(21), 4549 (1998)

[8] Roy, A., Lin, C.-Y., Mohideen, U.: Improved precision measurement of the Casimir force. Phys. Rev. D 60(11), 111101 (1999)

[9] Bordag, M., Klimchitskaya, G.L., Mohideen, U., Mostepanenko, V.M.: New developments in the Casimir effect. Phys. Rep. 353(1-3), 1-205 (2001)

[10] Bordag, M., Klimchitskaya, G.L., Mohideen, U., Mostepanenko, V.M.: Advances in the Casimir effect. Oxford University Press, Oxford (2009)

[11] Elizalde, E., Romeo, A.: Expressions for the zeta-function regularized Casimir energy. J. Math. Phys. 30(5), 1133-1139 (1989)

[12] Elizalde, E., Romeo, A.: Heat-kernel approach to the zeta-function regularization of the Casimir energy for domains with curved boundaries. Int. J. Mod. Phys. A 5(09), 1653-1669 (1990)

[13] Kirsten, K.: Spectral Functions in Mathematics and Physics. Chapman \& Hall/CRC, Boca Raton (2002)

[14] Brown, L.S., Maclay, G.J.: Vacuum stress between conducting plates: an image solution. Phys. Rev. 184(5), 1272 (1969)

[15] Dzyaloshinskii, I.E., Lifshitz, E.M., Pitaevskii, L.P.: General theory of Van der Waals' forces. Physics-Uspekhi 4(2), 153-176 (1961)

[16] Deutsch, D., Candelas, P.: Boundary effects in quantum field theory. Phys. Rev. D 20(12), 3063-3080 (1979)

[17] Kay, B.S.: Casimir effect in quantum field theory. Phys. Rev. D 20(12), 3052 (1979)

[18] Scharf, G., Wreszinski, W.F.: On the Casimir effect without cutoff. Found. Phys. Lett. 5(5), 479-487 (1992)

[19] Fulling, S.A.: Vacuum energy as spectral geometry. Sym. Integrab. Geom.: Meth. Appl 3, 094 (2007)

[20] Dappiaggi, C., Nosari, G., Pinamonti, N: The Casimir effect from the point of view of algebraic quantum field theory. arXiv preprint arXiv:1412.1409, (2014)

[21] Johnson, S.G.: Numerical methods for computing Casimir interactions. In: Dalvit, D., Milonni, P., Roberts, D., da Rosa, F. (eds.) Casimir Physics, pp. 175-218. Springer, Berlin (2011)

[22] Reid, M.T.H., Rodriguez, A.W., White, J., Johnson, S.G.: Efficient computation of Casimir interactions between arbitrary $3 d$ objects. Phys. Rev. Lett. 103(4), 040401 (2009)

[23] Rodriguez, A.W., Capasso, F., Johnson, S.G.: The Casimir effect in microstructured geometries. Nat. Photon. 5(4), 211-221 (2011) 
[24] Bimonte, G., Emig, T., Kardar, M., Krüger, M.: Nonequilibrium fluctuational quantum electrodynamics: heat radiation, heat transfer, and force. Ann. Rev. Condens. Matter Phys. 8, 119-143 (2017)

[25] Emig, T., Graham, N., Jaffe, R.L., Kardar, M.: Casimir forces between arbitrary compact objects. Phys. Rev. Lett. 99(17), 170403 (2007)

[26] Emig, T., Graham, N., Jaffe, R.L., Kardar, M.: Casimir forces between compact objects: the scalar case. Phys. Rev. Lett. 77(2), 025005 (2008)

[27] Emig, T., Jaffe, R.L.: Casimir forces between arbitrary compact objects. J. Phys. A: Math. Theor. 41(16), 164001 (2008)

[28] Emig, T., Jaffe, R.L., Kardar, M., Scardicchi, A.: Casimir interaction between a plate and a cylinder. Phys. Rev. Lett. 96(8), 080403 (2006)

[29] Kenneth, O., Klich, I.: Opposites attract: a theorem about the Casimir force. Phys. Rev. Lett. 97, 060401 (2006)

[30] Kenneth, O., Klich, I.: Casimir forces in a T operator approach. Phys. Rev. B 78, 014103 (2008)

[31] Milton, K.A., Wagner, J.: Multiple scattering methods in Casimir calculations. J. Phys. A: Math. Theor. 41, 155402 (2008)

[32] Rahi, S.J., Emig, T., Graham, N., Jaffe, R.L., Kardar, M.: Scattering theory approach to electrodynamic Casimir forces. Phys. Rev. D 80(8), 085021 (2009)

[33] Renne, M.J.: Microscopic theory of retarded van der Waals forces between macroscopic dielectric bodies. Physica 56, 125-137 (1971)

[34] Hanisch, F., Strohmaier, A., Waters, A.: A relative trace formula for obstacle scattering. arxiv: pdf/2002.07291 (2020)

[35] Balian, R., Duplantier, B.: Electromagnetic waves near perfect conductors II. Casimir effect. Ann. Phys. 112, 165-208 (1978)

[36] Kirsten, K., Lee, Y.: Gluing formula for Casimir energies. Symmetry 10(1), 31 (2018)

[37] Gies, H., Langfeld, K., Moyaerts, L.: Casimir effect on the worldline. JHEP 06, 018 (2003)

[38] Candelas, P.: Vacuum energy in the presence of dielectric and conducting surfaces. Ann. Phys. 143(2), 241-295 (1982)

[39] Garabedian, P.R., Schiffer, M.: Convexity of domain functionals. In Journal d'Analyse Mathématique 2, 281-368 (1952)

[40] Hadamard, J Mémoire Sur Le Problème D'analyse Relatif À L'équilibre Des Plaques Élastiques Encastrées. Mémoires des Savants Etrangers, 1908

[41] Peetre, J.: On Hadamard's variational formula. J. Differ. Equ. 36(3), 335-346 (1980)

[42] Strohmaier, A., Waters, A.: Geometric and obstacle scattering at low energy. Comm. Partial Differ. Equ. 45, 1451-1511 (2020)

[43] Shubin, M.A.: Pseudodifferential Operators and Spectral Theory, 2nd edn. Springer, Berlin (2001)

[44] Taylor, M.E.: Pseudodifferential Operators. Princeton Mathematical Series, vol. 34. Princeton University Press, Princeton (1981)

[45] McLean, W.: Strongly Elliptic Systems and Boundary Integral Equations. Cambridge University Press, Cambridge (2000) 
[46] Strohmaier, A., Verch, R., Wollenberg, M.: Microlocal analysis of quantum fields on curved space-times: analytic wave front sets and Reeh-Schlieder theorems. J. Math. Phys. 43(11), 5514-5530 (2002)

[47] Dyatlov, S, Zworski, M.: Mathematical Theory of Scattering Resonances. http:// math.mit.edu/ dyatlov/res/res_final.pdf, (2019)

[48] Henry, D.: Perturbation of the Boundary in Boundary-Value Problems of Partial Differential Equations. London Mathematical Society Lecture Note Series (318), Cambridge University Press, Cambridge (2005)

[49] Hezari, H., Zelditch, S.: $C^{\infty}$ Spectral rigidity of the ellipse. Analysis \& PDE 5(5), 1105-1132 (2012)

[50] Ozawa, S.: Hadamard's variation of the Green kernels of heat equations and their traces I. J. Math. Soc. Jpn. 34(3), 455-473 (1982)

[51] Suzuki, T., Tsuchiya, T.: First and second Hadamard variational formulae of the Green function for general domain perturbations. J. Math. Soc. Jpn. 68(4), 1389-1419 (2016)

[52] Chandler-Wilde, S.N., Graham, I.G., Langdon, S., Spence, E.A.: Numericalasymptotic boundary integral methods in high-frequency acoustic scattering. Acta Numer. 21, 89-305 (2012)

[53] Borisov, N.V., Müller, W., Schrader, R.: Relative index theorems and supersymmetric scattering theory. Comm. Math. Phys. 114(3), 475-513 (1988)

[54] Branson, T.P., Gilkey, P.B.: The asymptotics of the Laplacian on a manifold with boundary. Comm. Partial Differ. Equ. 15, 245-272 (1990)

[55] Greiner, P.: An asymptotic expansion for the heat equation. Arch. Rat. Mech. Anal. 41, 163-218 (1971)

[56] Gilkey, P.B., Smith, L.: The eta invariant for a class of elliptic boundary value problems. Comm. Pure Appl. Math. 36, 85-131 (1983)

[57] Kac, M.: On some connections between probability theory and differential and integral equations. In: Proceedings of Second Berkeley Symposium on Mathematical Statistics and Probability, pp. 189-215. University of California Press, Berkeley and Los Angeles, (1951)

[58] Li, L., Strohmaier, A.: Heat kernel estimates for general boundary problems. J. Spectr. Theory 6(4), 903-919 (2016)

[59] Jensen, A., Kato, T.: Asymptotic behavior of the scattering phase for exterior domains. Comm. Partial Differ. Equ. 3(12), 1165-1195 (1978)

[60] Kato, T.: Monotonicity theorems in scattering theory. Hadron. J. 1, 134-154 (1978)

[61] Gilkey, P.B.: Invariance Theory, the Heat Equation, and the Atiyah-Singer Index Theorem. Studies in Advanced Mathematics, 2nd edn. CRC Press, Boca Raton (1994)

Yan-Long Fang and Alexander Strohmaier

School of Mathematics

University of Leeds

Leeds, Yorkshire LS2 9JT

UK

e-mail: Y.L.Fang@leeds.ac.uk;

a.strohmaier@leeds.ac.uk 
Communicated by Karl-Henning Rehren.

Received: April 23, 2021.

Accepted: September 28, 2021. 